\title{
Systématique des Corégones de l'Europe occidentale, basée sur une étude biométrique par
}

\section{E. DOTTRENS}

Muséum d'histoire naturelle, Genève.

Travail exécuté avec l'appui du Fonds national de la recherche scientifique.

Avec 12 figures et 16 tableaux dans le texte

Le présent travail est un essai d'apporter une clarté nouvelle sur la systématique des Corégones d'Europe (Europe à l'exclusion de la Finlande et de la Russie où je n'ai pas eu l'occasion de me rendre). Il portait à l'origine, essentiellement, sur les Corégones de l'Europe centrale, ce n'est que secondairement que j'ai été amené à m'intéresser aux Corégones de Grande Bretagne et à ceux de Scandinavie.

On trouvera dans les travaux de Wagler et de Steinmann des historiques de la question et toute une littérature (in Auswahl !) sur lesquels je ne reviendrai guère. Ces deux auteurs ont défendu, non sans frictions, des opinions presque diamétralement opposées, je poserai donc le problème en résumant et en critiquant leurs conceptions.

\section{LE SYSTÈME DE WAGLER}

WaGLer (1937, 1941 et 1950) admet l'existence de 4 espèces de Corégones qui auraient pénétré en Europe centrale venant du N.-E. par des communications fluviales anciennes qu'il imagine dans la région des Sudètes, entre des affluents du Danube (March et Stille Adler) et de l'Oder (Glatzer Neisse). Je ne suis pas en mesure d'apprécier la validité de ces vues. Je signalerai seulement 
que j'ai vainement tenté d'obtenir des renseignements d'ordre géologique qui expliqueraient comment les Corégones auraient pu, naturellement, accéder dans le bassin du Rhône après les dernières glaciations. A plus forte raison, suis-je obligé à un scepticisme complet quant aux explications fournies sur l'apparition successive des diverses formes reconnues dans un bassin fluvial donné.

WAGLER reconnaît 4 espèces:

$1^{\circ}$ Coregonus wartmanni Bloch, Blaufelchen ou Grosse Schwebrenke, le grand Corégone de pleine eau.

$2^{\circ}$ Coregonus macrophthalmus Nüsslin, Gangfisch ou kleine Schwebrenke, le petit Corégone de pleine eau.

$3^{\circ}$ Coregonus fera Jurine, Sandfelchen ou grosse Bodenrenke, le grand Corégone de fond.

$4^{\circ}$ Coregonus acronius Rapp, Kilch ou kleine Bodenrenke, le petit Corégone de fond.

Dans certains lacs comme celui de Constance (Bodensee), les quatre espèces cohabitent et sont assez faciles à reconnaître; ailleurs, leur distinction est délicate et WAGLER fait intervenir les particularités biologiques pour les déterminer, ce qui est certainement judicieux, mais parfois terriblement sujet à caution. WAGLER en particulier attribue une importance majeure à la capacité de croissance (Abwachs), caractère différentiel pour lui primordial, dont il affirme même qu'il est peut-être le seul qui permette la distinction des espèces lorsque les individus ont atteint une certaine taille. «Vielleicht das einzige (Merkmal), das bei einer gewissen Grösse vorliegenden Exemplare die Unterscheidung der Arten gestattet». Cette conception s'oppose d'évidence au fait bien connu que la croissance des poissons en général est fonction de leurs conditions d'existence. Les expériences de W. Wunder (1939) sur les Carpes sont particulièrement frappantes à ce sujet.

WAGLER, se fondant sur l'appréciation des âges individuels par la lecture des écailles, déclare donc que la croissance est une constante d'une espèce donnée et qu'à température égale elle est identique au millimètre près pour chaque classe d'âge à l'intérieur de l'espèce. Cette affirmation ne résiste pas à l'examen, elle ressemble fort à une pétition de principe, les preuves qu'il en donne reposant sur une prémisse qu'il s'agirait d'abord de démontrer. La difficulté d'apprécier l'âge exact d'un poisson par le dénombrement des 
cercles de croissance des écailles — du fait que les vicissitudes de l'existence d'un individu peuvent se marquer sous forme de stries simulant des zones annuelles - rend en effet incertaine et parfois toute subjective une conclusion fondée sur une telle appréciation. D'ailleurs Einsele (1943) a montré à quelles erreurs elle peut conduire lorsqu'on prétend reconstituer les tailles qu'un exemplaire a mesurées aux différents âges de son existence. La simple observation montre que ces tailles annuelles recalculées selon la méthode usuelle sont d'autant plus faibles, pour les premières années surtout, que l'exemplaire est plus âgé. J'ai employé cette méthode au début, mais j'y ai renoncé, à cause de la part de subjectivité qu'elle comporte.

Les preuves que la potentialité de croissance n'a pas la valeur que Wagler lui attribue ne manquent pas, même chez les Corégones. Svärdson (1949 et 1951) en a fourni des exemples topiques.

Mais déjà ZANDT (1937 et 1950), par exemple, observait que le poids moyen et la croissance du Blaufelchen dans le lac de Constance augmentent quand la population du lac diminue et inversément.

Les recherches de Nümann (1938) aboutissent aux mêmes conclusions.

WAGLER connaissait ces observations mais il les mettait en doute affirmant par exemple qu'il n'existait pas de Corégone de profondeur dont la croissance fût forte. Il écrivait: «Weshalb nicht dann und wann ein gut wachsender Bodentierfresser in der Tiefe und ein langsam wüchsiger in den seichten Seeteilen ?»

On aurait pu lui citer en tout cas la Féra du Léman, Coregonus fera Jurine, telle que Fatro l'a encore connue et décrite, Corégone se nourrissant aux dépens de la faune de fond et qui frayait principalement en profondeur et jusqu'à $200 \mathrm{~m}$. Au début de ce siècle, les exemplaires pesant plusieurs kilos n'étaient pas rares, le Muséum de Genève en possède un qui accusait 4.700 grammes. Ces prises étaient faites à l'époque où l'espèce se raréfiait dangereusement. Dans le lac d'Aiguebelette, en Savoie, où le Lavaret subsiste péniblement à cause de l'eutrophie croissante, le poids moyen des individus atteint presque un kilo, il s'agit pourtant, comme on le verra plus loin, d'un Gangfisch (d'ailleurs mâtiné si on ose dire), malgré l'affirmation de WAGLER que cette forme n'existe pas dans les régions les plus occidentales de l'aire de répartition des Corégones. 
WagLer donnait la priorité au caractère de croissance sur le caractère nombre de branchiospines, il attribuait aussi une importance majeure à la biologie des populations, mœurs reproductrices, biotopes préférés, nourriture habituelle. C'était méconnaître que le nombre de branchiospines est le seul caractère dont la fixité génétique soit démontrée. Il fut ainsi amené à qualifier délibérément de Gangfisch le poisson du Chiemsee dont la moyenne du nombre des branchiospines du premier arc est d'environ 25, moyenne caractéristique des Sandfelchen, alors que celle des Gangfisch, supérieure à celle des Blaufelchen même, est voisine de 40. Cette façon autoritaire d'interpréter les faits diminue sensiblement la valeur des tabelles où WAGLER indique pour de nombreux lacs d'Europe centrale la présence ou l'absence des espèces reconnues par lui.

\section{La conception de Steinmann}

A l'opposé de Wagler, Steinmann (1948 et 1950) se rallie à l'opinion que tous les Corégones autres que Coregonus albula se rattachent à une seule espèce Coregonus lavaretus. Il affirme que ces Corégones se sont diversifiés dans les temps géologiques les plus récents en une série de formes convergentes, le phénomène se manifestant de nos jours encore. Chaque regio, lac ou groupe de lacs d'un même bassin fluvial, hébergerait donc une natio qui lui est propre. Il reconnaît en Suisse les regiones suivantes: rhodanensis (du Rhône), arurensis (de l'Aar) jurassica (lacs du pied du Jura dont les émissaires aboutissent à l'Aar), riusensis (de la Reuss, affluent de l'Aar), lindimacensis (de la Limmat, affluent de l'Aar), bodanica (du Bodensee ou Bodan, lac de Constance) et enfin intermedia pour l'ensemble des bassins tributaires de l'Aar et du Rhin qui n'entrent pas dans les groupes précédents (lacs de Sempach, Baldegg, Hallwill et Greifensee).

Dans chaque natio, et sur place, l'unique forme originelle se serait scindée en écotypes: littoralis, pelagicus, profundus, nanus, la forme primigenius seule ayant conservé les caractères ancestraux. Il résulterait de cette conception que deux formes sympatriques nettement dissemblables seraient plus proches parentes étant de souche unique que dans deux lacs différents deux formes indiscernables qui ne seraient que des convergences dues à l'adaptation à 
des milieux semblables. Le Blaufelchen du lac de Constance serait alors proche parent du Kilch, il ne serait qu'analogue au Blaufelchen du lac de Zurich (Albeli).

Cette interprétation ne saurait satisfaire la plupart des biologistes actuels qui admettent qu'une barrière - en principe géographique — doit séparer durablement deux populations d'une même espèce pour qu'elles acquièrent à la longue une autonomie reproductrice telle qu'elles peuvent ensuite se maintenir sympatriquement. Steinmann fut conscient de cette difficulté; du moins il admettait bien la nécessité d'une barrière et il pensait justement l'avoir trouvée dans la fidélité des poissons grégaires au banc qu'ils ont formé à leur sortie de l'œuf. Pour lui, chaque banc de Corégones est une «Sippe», un groupe d'individus étroitement apparentés, formé lors de l'éclosion sur la frayère et qui maintient pratiquement sa cohésion tout au long de son existence: «Gesellchaften auf Lebenszeit, die sich wohl gelegentlich trennen und wieder vereinigen können, zu denen wahrscheinlich zuweilen auch Zuzügler stossen, die aber alles in allem doch aus Geschwistern bestehen und somit Inzuchtgesellschaften bilden ».

L'idée que Sternmann se faisait des «Sippen» expliquerait à la rigueur le maintient de divers écotypes dans un même bassin, elle ne rend pas compte de leur origine, puisqu'à l'arrivée des primigenius dans un lac ils avaient par définition des habitudes communes et la même amblivalence pour les divers biotopes. Au début au moins, cette absence de différenciation s'opposait à une ségrégation en écotypes. On a l'impression qu'une arrière-pensée lamarckienne d'adaptation au milieu règne dans le système de Steinmann. La barrière nécessaire à l'isolement reproducteur était donc pour cet auteur un instinct grégaire assez exclusif pour aboutir à des sociétés pratiquement fermées. Il admettait encore que l'adaptation aux conditions du milieu et la formation d'écotypes procédaient avec une étonnante rapidité. La Féra actuelle du Léman, dit-il, n'a plus les mêmes mœurs reproductrices qu'à l'époque où FAtio distinguait encore Coregonus fera schinzi, de la Gravenche, Coregonus hiemalis. La Féra se serait donc modifiée écologiquement depuis 50 ans. Sternmann envisageait bien l'éventualité d'un mélange avec la Palée du lac de Neuchâtel (introduite en masse dès 1923), mais il classe cette "heutige Fera » Coregonus lavaretus rhodanensis primigenius. Or je pense avoir 
établi (Dottrens, 1950) que le Corégone actuel du Léman n'est rien autre que la Palée introduite au moment où les anciennes formes autochtones périclitaient. Si tel est bien le cas, ce Corégone actuel appartient à la natio jurassica et non pas rhodanensis. Si, contre toute apparence, la véritable Féra autochtone existe encore, elle ne doit pas s'appeler dans le système de Sternmann primigenius, mais, avec ses mœurs reproductrices (fraie à grande profondeur en février), profundus ou plutôt, à cause de ses habitudes nutritives (faune de fond prélevée sur la beine à la belle saison) et de ses 25 branchiospines en moyenne, littoralis.

Bien que faisant des allusions occasionnelles à des mélanges et aux possibilités d'hybridations naturelles, SteInmann, pas plus que WAGLER, ne reconnaît explicitement des populations hybrides. Il aboutit en suivant fidèlement son idée des «Sippen» isolées génétiquement à des conclusions désarmantes. Dans le lac de Zoug, Fatio (1890) distinguait sans peine le Balchen à 19-24 Branchiospines, Coregonus schinzi helveticus (un Sandfelchen) et l'AlbeliAlbock avec 35-39 branchiospines qu'il rapportait à Coregonus wartmanni, au Blaufelchen donc. Depuis l'époque de Fatio, des immersions multiples ont eu lieu dans ce lac avec du frai de provenances diverses. La population actuelle présente une telle variabilité que la distinction des diverses formes autochtones ou introduites y est impossible de l'avis de Sternmann lui-même. Mais au lieu de conclure à un mélange inextricable, cet auteur déclare que les écotypes s'y sont encore trop peu consolidés: «Eine Unterteilung ist zur Zeit noch unmöglich, da sich die Ökotypen noch zu wenig konsolidiert haben». Le Balchen de Zoug serait donc: Coregonus lavaretus nat. riusensis ecot. primigenius et l'Albeli, forme éteinte, entre péniblement dans le système sous le nom de Coregonus lavaretus riusensis primigenius nanus.

Le nombre des branchiospines d'après Steinmann varie entre 21 et 36 pour le Balchen actuel et 24-28 pour l'Albeli éteint (FAtio, respectivement, 19-24 et 35-39 branchiospines).

J'ai procédé moi-même à des mensurations sur un contingent de ce Corégone du lac de Zoug. J'ai trouvé sur 69 individus une moyenne de 27,35 branchiospines et la dispersion considérable des formes hybrides ou mélangées. Même pour le lac de Sempach, où il qualifie le Corégone actuel de mixtum compositum, Sternmann classe ce Corégone comme Coregonus lavaretus natio intermedius 
ecot. primigenius avec 26 à 38 branchiospines alors que FAtio avait trouvé 38 à 42 branchiospines, ce qui correspond à un Gangfisch indubitable; mais FAтіо en avait fait une espèce à part et mème une species composita sous le nom de Coregonus suidteri Fatio parce qu'il prenait en considération des proportions particulières du corps, des nageoires et des branchiospines.

Les idées de Sternmann sont contredites par des faits aisément contrôlables. Ainsi, Svärdson cite des expériences de marquages prouvant que les Corégones ne sont pas absolument fidèles à leurs emplacements de fraie. Il est en outre facile d'observer que sur une frayère des individus de tailles et d'âges très différents sont réunis. Les bancs d'âges divers s'y mélangent donc au moment de la reproduction. Par exemple, au bord du lac de Thoune, à Merlingen, on peut assister sans difficulté à la fraie des Balchen et observer que les couples qui parfois s'élancent hors de l'eau peuvent être formés d'individus de tailles très dissemblables. Il s'agit de Coregonus schinzi d'après FAtio, nous verrons plus loin qu'il faut le rapporter à la troisième espèce reconnue par biométrie. J'ai eu l'occasion de constater l'instabilité des bancs de Corégones dans le Tegernsee, en Bavière. Le 20 mai 1955, les filets de fond du pêcheur KELLER, titulaire exclusif du droit de pêche dans ce lac, prenaient 30 exemplaires, soit 23 femelles et 7 mâles. Le 21 mai, il y eut 20 captures par les filets de fond: 15 femelles et 5 mâles. Ce même jour, à un tout autre endroit du lac, les aides du pêcheur ramenaient à l'aide de filets flottants 34 individus, soit 7 femelles et 27 mâles. Ainsi donc, à ce moment, la plupart des femelles se tenaient près $\mathrm{du}$ fond, la plupart des mâles étaient en pleine eau et sur des emplacements différents. Un orage survint dans la la nuit du 21 au 22 mai. Les jours suivants on capturait des mâles et des femelles en nombre égal tant au filet de fond qu'au filet flottant. Donc, au gré des conditions, atmosphériques dans le cas présent, les bancs se font et se défont, ils se désagrègent et se reconstituent selon les circonstances et rien ne permet de supposer ou d'affirmer que ces mouvements se font sans chassés-croisés. Dans le cas du Tegernsee, les individus capturés sur le fond appartiennent à la même forme, relativement pure, que ceux qui sont pris en pleine eau. Il ne s'agit absolument pas d'écotypes différents, tout au plus les contingents pris au fond revèlent-ils pour une allure semblable de la courbe de variabilité du nombre de bran- 
chiospines une certaine irrégularité révélatrice d'un probable mélange, sans doute par apport artificiel d'une petite proportion d'individus d'origine étrangère. Il ne faut, en effet, jamais oublier que les fantaisies de la pisciculture, presque partout, en Europe centrale en tout cas, masquent plus ou moins gravement la simplicité originelle des faits. C'est pourquoi, soit dit entre parenthèses, la systématique des Corégones d'Europe centrale ne saurait être dorénavant qu'une reconstitution, et que le praticien, s'il méconnaît l'influence perturbatrice des pratiques piscicoles, trouvera toujours des exemples confus à opposer aux conclusions du systématicien.

Dans ces cas de brassage par interventions humaines, le systématicien a le droit et même le devoir de se récuser. Je pense apporter plus loin, par des calculs statistiques de variabilité et de dispersion, des preuves parfaitement objectives des complications résultant des transplantations, immersions, acclimatations et autres «rajeunissements du sang» pratiqués par les pisciculteurs. La situation dans certains lacs en est à tel point obscurcie qu'elle est devenue inextricable. Dans ces cas-là, il n'est plus question de systématique, tout au plus de classement de formes remaniées par l'homme, formes comparables aux races de carpes d'élevage par exemple, à cela près qu'elles ne résultent pas d'une sélection artificielle dirigée.

Le système de Steinmann est fondé sur l'affirmation que Coregonus lavaretus est parvenu dans les lacs d'Europe centrale sous la forme primigenius qui se serait maintenue dans un certain nombre de bassins. Le type de primigenius provient du lac de Constance. Steinmann en a publié la photographie dans un article de 1948.

Il s'agit d'un jeune Corégone de 29 cm, 230 gr, 4 ans, capturé le 4 décembre 1947. Ni dans sa monographie, ni dans cet article, l'auteur ne donne le nombre de branchiospines de ce type qui n'a pas été conservé puisqu'il n'existe pas dans la collection STEIn mann acquise par le musée de Bâle. J'ai relevé ailleurs (Dоттrens, 1953) le fait que les individus retenus par l'auteur comme appartenant à ce même écotype dans le lac de Constance, se rapportent d'évidence à un mélange de deux formes, les plus abondants, avec un mode du nombre des branchiospines égal à 36, accusent la moyenne même des Blaufelchen de ce lac. Les autres dénombrements et mensurations fournis montrent d'évidence que cette forma primigenius n'a pas toujours d'existence objective. Nous avons vu 
qu'au lac de Zoug elle n'est rien d'autre qu'un produit d'hybridations artificielles et empiriques.

\section{La confusion de la systématique des Corégones}

On vient de voir que la confusion résulte en partie des manipulations de l'homme. Pour ce qui concerne les interventions relativement récentes, j'ai montré (Dotтrens 1955) que les transplantations de Corégones d'origines diverses ont abouti, dans le lac Majeur du nord de l'Italie et dans le lac d'Annecy en Haute-Savoie à la création de populations hybrides stables auxquelles il est vain, me semble-t-il, de donner un nom comme le fit R. Monti (1929), lorsque cet auteur prétendit avoir trouvé dans les formes particulières aux lacs italiens la preuve de la plasticité des Corégones. Mais ces manipulations de l'homme pourraient bien être beaucoup plus importantes et plus anciennes qu'on ne l'admet d'ordinaire. Dans bien des cas difficiles, la supposition la plus simple et partant la plus plausible quant à l'origine de certaines populations serait peut-être d'attribuer aux moines l'acclimatation des Corégones dans certains lacs. Ce n'est peut-être pas une simple coïncidence, si un Corégone existe en France dans le lac du Bourget et que justement au bord de ce même lac se dresse l'antique abbaye de Hautecombe. Du moins, la supposition que les moines auraient pu être à l'origine d'acclimatations anciennes ne me paraît pas toutà-fait gratuite. WAGLER ne cite-t-il pas le cas de l'abbé Wilhelm von Benediktbeuren qui, en 1480, introduisit des Corégones dans le Walchensee et Thienemann ne fait-il pas allusion à une inscription runique de Norvège, datant de l'an mille environ, relative à une introduction artificielle de poisson dans un certain lac Roud ?

Pour le Léman, on a des preuves fort intéressantes et bien curieuses de l'intérêt économique des Corégones et de la valeur que leur attribuaient les religieux.

Ce sont deux documents cités par F. A. Forel (1904).

Le premier date de 1376, c'est la taxe des poissons de Villeneuve, qui fixe les prix de vente de la livre de Féra «libram ferratarum» et celui de la livre de Bezole «libram bisolarum». ForeL admet sans hésiter que ferrata ne peut être que Coregonus fera Jurine et pense que bisola ou Bezole est la Gravenche, Coregonus hiemalis. C'est loin d'être évident; en effet, le second document, 
datant de 1150, fixe la contribution que le prieuré de Saint-Jean à Genève devait fournir pour la table des chanoines d'Aoste dont il dépendait. Le prieuré devait procurer, entre autres vivres, des palatae ou, à défaut, des ferratae. Il fallait 4 ferratae pour équivaloir à une pala. $\mathrm{Au} \mathrm{xII}^{\mathrm{e}}$ siècle donc, la Féra (ferra ou ferrata) était le p e tit Corégone, le grand Corégone du Léman était alors la Palée (!), pala ou palata et non pas bisola comme deux siècles plus tard à l'autre bout du lac. De nos jours encore, au lac du Bourget, Bezole est le nom d'un Corégone plus grand que le Lavaret, que Fатіо caractérisait par un nombre moindre de branchiospines et soupçonnait d'être un produit d'hybridation. La Bezole est actuellement indiscernable et le Lavaret est certainement hybridé, comme nous le verrons plus loin.

Il apparaît bien que deux formes au moins existaient autrefois dans les lacs du bassin du Rhône, une plus petite, l'ancienne ferra au Léman et le Lavaret au Bourget, et une plus grande, l'ancienne pala du Léman et la Bezole au Bourget. Le nom de Féra au Léman aurait passé d'une forme à l'autre tandis que se perdait celui de Bezole. Ces conclusions paraitront peut-être tirées par les cheveux, elles semblent pourtant confirmées par une curieuse constatation de Spillmann au muséum de Paris. Cet auteur a examiné les deux exemplaires de Corégones, étiquetés Coregonus fera Jurine provenant du Léman, qui par chance portent encore leur étiquette de parchemin d'origine. L'un, $411 \mathrm{~mm}$, provient de la collection Moreau, l'autre, 267 mm seulement, de la collection Kiener 1828. Ils ont respectivement 40 et 41 branchiospines au premier arc; ce sont des nombres typiques de Gangfisch (Coregonus exiguus Nusslin in Fatio). Fatio à la fin du xixe siècle ne reconnaissait pas le Gangfisch au Léman, il soupçonnait par contre la Gravenche, Coregonus hiemalis Jurine et la Bezole du Bourget d'être des hybrides avec des formes disparues. J'ai critiqué cette supposition, je suis convaincu maintenant que j'ai eu tort. L'histoire des Corégones du bassin du Rhône s'avère plus compliquée qu'on aurait pu le soupçonner. La difficulté, dans ce cas et vraisemblablement dans bien d'autres, ne parait pas celle d'un simple problème de systématique. Il est permis de supposer qu'en fouillant les archives des antiques abbayes qui ont possédé des domaines au bord des lacs on retrouverait des indices d'anciennes interventions humaines qui pourraient rendre compte de certaines difficultés actuelles de 
la systématique de notre groupe. De toute façon, les Corégones ne sont pas un matériel exemplaire pour l'étude des relations naturelles des êtres avec leur milieu, et des phénomènes d'adaptation.

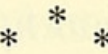

Les systèmes proposés par les ichthyologues pour la nomenclature des Corégones fluctuent depuis longtemps entre deux extrêmes. Les uns, avec Linné admettent deux espèces: albula, la petite Marène et lavaretus (on y ajoute parfois $C$. oxyrhynchus (L.)). Les autres multiplient les espèces et les sous-espèces à l'infini. Les descriptions de Fatio (1890) sont un exemple de cette tendance. L'auteur a été d'évidence leurré par l'emploi de caractères morphologiques dépendant des conditions d'existence, dépourvus de valeur systématique, parce que non héréditaires. Ceux qui rapportent toutes les populations sauf celles de Coregonus albula à la seule espèce lavaretus, quitte à la pulvériser en une multitude de sous-espèces et d'écotypes, me paraissent méconnaître le fait fondamental de l'existence sympatrique de quelques types, les 4 espèces de Wagler ou peut-être les 5 écotypes de Steinmann, aisément reconnaissables et distincts quand les interventions humaines n'ont pas trop bouleversé les conditions naturelles. Quelle que soit la facilité avec laquelle ces formes se laissent hybrider par fécondation artificielle, c'est un fait qu'elles sont pratiquement isolées les unes des autres quand elles cohabitent dans un bassin donné. Dans la région alpine, on constate aisément que les bons pêcheurs les reconnaissent sans hésitation au moins au moment de la capture. Cette sûreté de coup d'œil est frappante justement dans les lacs où la pisciculture n'a pas compliqué la situation et contraste avec l'embarras des mêmes professionnels dans les lacs où on a procédé à d'abondantes immersions d'alevins destinés à « rajeunir le sang»!

Cependant les interventions humaines n'expliquent pas tout et dans beaucoup de cas, il faut bien, semble-t-il, admettre divers degrés d'introgression naturelle d'une forme dans une autre, mélanges partiels qui témoignent de la fragilité relative des barrières qui s'opposent normalement à l'hybridation. Les Corégones paraissent appartenir à un type d'espèces capables de former des hybrides sous certaines conditions, mais aptes tout de même à se 
maintenir sympatriquement par le jeu de mécanismes isolateurs qui ne sont pas forcément des incompatibilités géniques ou chromosomiques et qui peuvent être, par exemple, des habitudes de fraie décalées dans le temps ou dans l'espace. On peut supposer que ces possibilités d'hybridation naturelle ne sont pas égales entre les diverses espèces, ni même pour les différentes populations d'une même espèce.

Des populations hybrides se sont formées dans le lac Majeur, en Italie et dans le lac d'Annecy en France. Pourtant, les souches des œufs et des alevins introduits se maintiennent distinctes dans les lacs d'origine. J'ai fait remarquer (Dottrens 1955) que dans ces deux lacs un temps de latence correspondant à un certain nombre de générations sépare les dates des transplantations de celles du succès manifeste des acclimatations. Il semble que l'hypothèse puisse être retenue d'une élimination progressive des facteurs d'isolement avant que la population hybridée s'épanouisse.

Le critère de l'isolement reproducteur par incompatibilités génique ou chromosomique, qui théoriquement devrait à mon sens définir une espèce, n'est donc pas ou pas complètement satisfait dans le genre Coregonus, il y a trop de populations dont les caractères plus ou moins aberrants - je parle surtout du nombre des branchiospines, le seul caractère dont la nature héréditaire soit démontrée — dont les caractères aberrants dis-je ne s'expliquent guère que par des hybridations plus ou moins anciennes ou par des phénomènes d'introgression. C'est du moins la conclusion où m'ont amené mes études biométriques. Il semble bien que les Corégones d'Europe représentent un «gene pool». On a proposé divers noms pour des cas analogues. Sonneborn (1957) lors d'un symposium récent suggère syngène, $\mathrm{V}$. Grant lors du même symposium reprend le terme de syngameon, créé par le botaniste Lotsy, qui désigne un complexe d'espèces capables de s'hybrider. Grant redéfinit ce terme: l'ensemble des espèces ou semi-espèces liées par une hybridation fréquente ou occasionnelle naturelles. Je pense qu'il faut considérer le genre Coregonus comme un complexe d'espèces incomplètement séparées, probablement parce que l'isolement au cours duquel elles ont amorcé leur diversification n'a pas été suffisamment prolongé, et qui subissent, surtout du fait de l'intervention humaine, un brassage aboutissant dans des cas particuliers à une réunification par hybridation. Les systèmes qui 
comme celui de BERG opposent $C$. lavaretus avec ses multiples subdivisions à $C$. albula me paraissent fautifs parce que contrairement à ce qu'ils sous-entendent le groupe albula n'est pas isolé génétiquement du groupe lavaretus. On peut obtenir des hybrides aussi entre ces deux types (Svärdson 1938) et l'analyse biométrique m'amène à reconnaître des cas d'introgression chez $C$. albula. M. Gasowska (1956) a établi que le croisement lavaretus $\times$ albula s'effectue sans difficulté, que la fécondation est normale, que les hybrides se développent et atteignent la maturité sexuelle comme les produits normaux. Le cas limite de Coregonus me paraît un exemple du fait que la nomenclature ne peut pas répondre fidèlement à tous les stades de l'évolution des formes. Etant un cas limite, il est d'évidence scabreux, en ce sens qu'il oblige à choisir. J'ai opté pour la solution taxonomique qui rend le mieux compte de la réalité des faits, qui est l'existence chez les Corégones d'Europe d'une demidouzaine de formes qui se comportent, au moins dans la plupart des cas, comme des entités, définies objectivement par les moyens statistiques de la biométrie, distinctes quoique voisines, sympatriques souvent deux à deux, bref des espèces.

\section{Premiers essais D'analyse}

Ayant à l'esprit les considérations qui précèdent et désireux d'éviter si possible de tomber dans l'arbitraire, j'ai tenté de distinguer par des moyens purement biométriques et statistiques les formes sympatriques et de reconnaître dans les différents bassins les formes ainsi caractérisées. Un tel travail me paraît la base indispensable à des études ultérieures, de nature écologique par exemple, qui infirmeront ou confirmeront la validité des types reconnus biométriquement. Après tant d'auteurs, j'ai été amené à reconnaître comme seules valables les particularités des branchiospines. Leur nombre surtout, dont on sait qu'il est fixé génétiquement, mais aussi leur longueur relative que j'ai exprimée d'abord par le nombre «d'écarts».

J'obtiens ce nombre d'écarts en reportant au moyen d'un compas fin la longueur de la plus grande branchiospine du premier arc sur la branche inférieure de cet arc. On peut compter aisément les intervalles ou écarts interspinaux à une demi-unité près. Dans le schéma (fig. 1), par exemple, je compte $41 / 2$. 
Dans les tableaux qui suivent, les longueurs moyennes (du museau à la pointe du lobe inférieur de la caudale) sont indiquées entre parenthèses lorsque les poissons ont été mesurés après fixation. On ne tient pas assez compte, me semble-t-il, dans les études biométriques, des effets sensibles de la fixation sur les mensura-

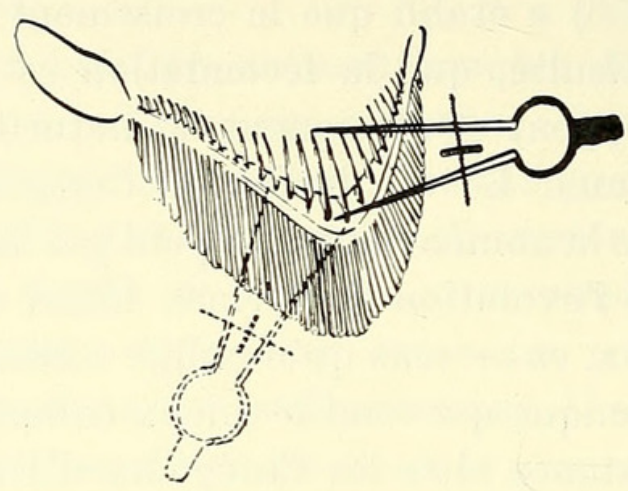

FIG. 1.

tions (voir à ce sujet KENNEDY 1943). La rétraction due au formol par exemple, peut atteindre presque 5\% selon les conditions; une telle rétraction rend presque illusoire les comparaisons de certains rapports de proportions calculés sur du matériel qui n'était pas absolument frais. J'ai vérifié pour la longueur du corps les effets de la fixation en comparant sur les mêmes individus les mesures prises sur matériel frais et refaites en laboratoire. Voici quelques exemples, les nombres donnés sont des moyennes:

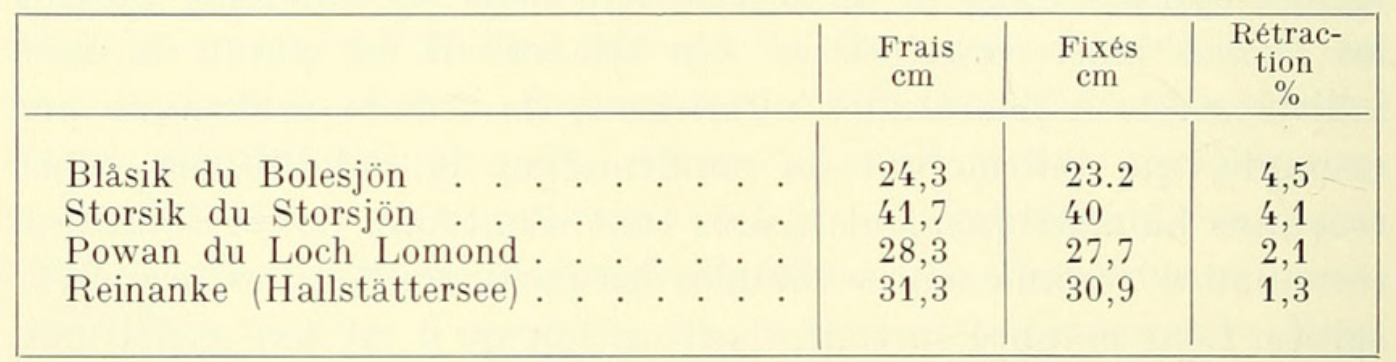

Je n'ai pas analysé les conditions qui déterminent l'importance de cette rétraction. La déshydratation des tissus entre la sortie de l'eau et la fixation joue un rôle évident, la concentration et la nature du fixateur également. Pour les deux premiers exemples cités, les poissons capturés en Suède du nord par quelque - 200 ont gelé sur le bateau même, ils ont dégelé à la température du 
TABLEAU 1

Corégones de Suisse, de France et d'Italie

\begin{tabular}{|c|c|c|c|c|c|c|c|}
\hline $\begin{array}{l}\text { No } \\
\text { d'or- } \\
\text { dre }\end{array}$ & $\begin{array}{l}\text { Population } \\
\text { (non usuel) }\end{array}$ & Lac & $\begin{array}{l}\text { Nom- } \\
\text { bre } \\
\text { d’indi- } \\
\text { vidus }\end{array}$ & $\begin{array}{l}\text { Nombre de } \\
\text { branchio- } \\
\text { spines }\end{array}$ & Ecarts & $\begin{array}{l}\text { Lon- } \\
\text { gueur } \\
\text { moy. } \\
\text { en cm }\end{array}$ & $\begin{array}{c}\text { Epoque } \\
\text { de capture }\end{array}$ \\
\hline $\begin{array}{r}61 \\
1\end{array}$ & $\begin{array}{l}\text { Kropfer } \\
\text { Kilch }\end{array}$ & $\begin{array}{l}\text { Thoune } \\
\text { Constance } \\
\text { (Untersee) }\end{array}$ & $\begin{array}{r}9 \\
70\end{array}$ & $\begin{array}{l}20,8 \pm 0,46 \\
21,1 \pm 0,17\end{array}$ & $\begin{array}{l}3,9 \pm 0,13 \\
3,5 \pm 0,08\end{array}$ & $\begin{array}{l}27 \\
27\end{array}$ & $\begin{array}{l}\text { avril } \\
\text { nov.-déc. }\end{array}$ \\
\hline $\begin{array}{c}\operatorname{sans} n^{\circ} \\
20\end{array}$ & $\begin{array}{l}\text { Féra vraie } \\
\text { Sandfelchen }\end{array}$ & $\begin{array}{l}\text { Léman } \\
\text { Constance }\end{array}$ & $\begin{array}{r}6 \\
89\end{array}$ & $\begin{array}{l}(24,3) \\
24,6 \pm 0,26\end{array}$ & $\begin{array}{l}(5,1) \\
\quad 4,5 \pm 0,08\end{array}$ & $\begin{array}{l}(52) \\
44\end{array}$ & $\begin{array}{l}\text { coll. de musée } \\
\text { nov. en fraie }\end{array}$ \\
\hline 19 & Weissfelchen & $\begin{array}{l}\text { Constance } \\
\text { (Untersee) }\end{array}$ & 345 & $25,0 \pm 0,13$ & $4,7 \pm 0,04$ & 38 & nov. et juin \\
\hline $\begin{array}{l}28 \\
49 \\
45 \\
29 \\
49 \\
30\end{array}$ & $\begin{array}{l}\text { "Féra actuelle } \\
\text { Palée } \\
\text { "Coregono } \\
\text { Palée = Balchen } \\
\text { Palée "de fond " } \\
\text { Balchen }\end{array}$ & $\begin{array}{l}\text { Léman } \\
\text { Neuchâtel } \\
\text { Vivarone } \\
\text { Bienne } \\
\text { Neuchâtel } \\
\text { Zoug }\end{array}$ & $\begin{array}{r}472 \\
557 \\
140 \\
86 \\
91 \\
69\end{array}$ & $\begin{array}{l}26,8 \pm 0,11 \\
26,9 \pm 0,10 \\
27,2 \pm 0,22 \\
27,5 \pm 0,34 \\
27,6 \pm 0,20 \\
27,4 \pm 0,35\end{array}$ & $\begin{array}{l}5,4 \pm 0,05 \\
5,3 \pm 0,09 * \\
5,1 \pm 0,05 \\
5,8 \pm 0,09 \\
5,5 \pm 0,07 \\
5,0 \pm 0,10\end{array}$ & $\begin{array}{l}44 \\
41 \\
30 \\
41 \\
41 \\
44\end{array}$ & $\begin{array}{l}\text { époques diverses } \\
\text { " } \\
\text { printemps } \\
\text { nov.-déc. } \\
\text { mi-déc. en fraie } \\
\text { juillet }\end{array}$ \\
\hline $\begin{array}{c}31 \\
\operatorname{sans}^{\circ}\end{array}$ & $\begin{array}{l}\text { Balchen } \\
\text { Gravenche }\end{array}$ & $\begin{array}{l}\text { Thoune } \\
\text { Léman }\end{array}$ & $\begin{array}{r}102 \\
6\end{array}$ & $\begin{array}{l}29,1 \pm 0,18 \\
(29,2)\end{array}$ & $\begin{array}{l}5,0 \neq 0,10 * \\
(5,7)\end{array}$ & $\begin{array}{l}33 \\
(35)\end{array}$ & $\begin{array}{l}\text { déc. en fraie } \\
\text { coll. de musée }\end{array}$ \\
\hline $\begin{array}{l}60 \\
24\end{array}$ & $\begin{array}{l}\text { "Lavaret" } \\
\text { Coregono bianco }\end{array}$ & $\begin{array}{l}\text { Annecy } \\
\text { Lac Majeur }\end{array}$ & $\begin{array}{l}509 \\
125\end{array}$ & $\begin{array}{l}31,6 \pm 0,14 \\
31,7 \pm 0,25\end{array}$ & $\begin{array}{l}7,2 \pm 0,05 \\
7,0 \pm 0,08\end{array}$ & $\overline{38}$ & $\begin{array}{l}\text { époques diverses } \\
\text { été }\end{array}$ \\
\hline $\begin{array}{l}32 \\
50 \\
33\end{array}$ & $\begin{array}{l}\text { Bondelle } \\
\text { Bondelle } \\
\text { Blaufelchen }\end{array}$ & $\begin{array}{l}\text { Bienne } \\
\text { Neuchâtel } \\
\text { Constance }\end{array}$ & $\begin{array}{l}536 \\
436 \\
193\end{array}$ & $\begin{array}{l}33,6 \pm 0,10 \\
34,0 \pm 0,10 \\
36,3 \pm 0,11\end{array}$ & $\begin{array}{l}6,8 \pm 0,08^{*} \\
7,6 \pm 0,08^{*} \\
7,6 \pm 0,05\end{array}$ & $\begin{array}{l}32 \\
31 \\
34\end{array}$ & $\begin{array}{l}\text { août, sept. et déc. } \\
\text { époques diverses } \\
\text { juin et déc. }\end{array}$ \\
\hline 16 & Blaufelchen & $\begin{array}{l}\text { Constance } \\
\text { (Untersee) }\end{array}$ & 61 & $36,7 \pm 0,27$ & $8,2 \pm 0,16$ & 40 & époques diverses \\
\hline $\begin{array}{l}17 \\
42\end{array}$ & $\begin{array}{l}\text { Lavaret } \\
\text { Gangfisch }\end{array}$ & $\begin{array}{l}\text { Bourget } \\
\text { Constance } \\
\text { (Obersee) }\end{array}$ & $\begin{array}{r}98 \\
122\end{array}$ & $\begin{array}{l}38,1 \pm 0,31 \\
38,4 \pm 0,26\end{array}$ & $\begin{array}{l}9,2 \pm 0,12 \\
8,9 \pm 0,07\end{array}$ & $\begin{array}{l}36 \\
28\end{array}$ & $\begin{array}{l}\text { printemps } \\
\text { juin et août }\end{array}$ \\
\hline $\begin{array}{l}22 \\
38 \\
23\end{array}$ & $\begin{array}{l}\text { Albock } \\
\text { Lavaret } \\
\text { Gangfisch }\end{array}$ & $\begin{array}{l}\text { Thoune } \\
\text { Aiguebelette } \\
\text { Constance }\end{array}$ & $\begin{array}{l}90 \\
75 \\
93\end{array}$ & $\begin{array}{l}38,3 \pm 0,22 \\
39,2 \pm 0,31 \\
40,8 \pm 0,21\end{array}$ & $\begin{array}{l}9,2 \pm 0,16 \\
9,7 \pm 0,13 \\
9,5 \pm 0,07\end{array}$ & $\begin{array}{l}38 \\
41 \\
29\end{array}$ & $\begin{array}{l}\text { déc. en fraie } \\
\text { été en fraie } \\
\text { déc. en }\end{array}$ \\
\hline 21 & Brienzlig & Brienz & 100 & $41,1 \pm 0,20$ & $9,7 \pm 0,1$ & 17 & juillet \\
\hline 55 & Corégone importé & $\begin{array}{l}\text { Chauvet } \\
\text { (Auvergne) }\end{array}$ & 22 & $46,2 \pm 0,42$ & $13 \pm 0,42$ & 21 & janv. et août \\
\hline
\end{tabular}

* Calculés sur 50 individus.

(Entre parenthèses, mesures prises sur individus fixés.) 
local où je les mesurais, ils ont repris en glace dès qu'ils ont été déposés à l'extérieur. On les a expédiés dans cet état de congélation après quelques semaines. On les a de nouveau dégelés à Genève et fixés au formol à 6\% environ.

Les Powans ont été fixés au formol une ou deux heures après capture, les Reinanken fixés à l'alcool à 70\% aussi après quelques heures. Il résulte de ces faits que la plus grande circonspection est de rigueur lorsqu'on veut comparer biométriquement des poissons frais et du matériel de musée. Dans les tableaux 1 et suivants, je donne avec les mesures les époques de capture, les périodes de fraie étant en principe le meilleur moment pour obtenir des contingents de formes pures, ce qui n'est d'ailleurs pas une garantie. C'est pourquoi, bien que ne faisant aucun tri préalable en principe, j'ai éliminé pour le Weissfelchen par exemple, des contingents pris en fraie en novembre, quelques Blaufelchen faciles à reconnaître au sortir de l'eau et bien des Gangfisch dont le nombre de branchiospines est assez différent pour qu'une erreur d'attribution soit exclue. Un tel tri n'est guère possible d'ordinaire et il vaut mieux en principe conserver sciemment des individus d'origine incertaine que les éliminer arbitrairement dans les calculs des moyennes.

On voit que si on tient compte à la fois du nombre des branchiospines et des écarts tels qu'ils ont été définis, ces diverses populations se groupent assez clairement comme l'indiquent les subdivisions du tableau 1. J'ai montré ailleurs (Dottrens 1955) que les populations no 61, prétendu Lavaret d'Annecy, et no 24, Coregono bianco du lac Majeur, sont des hybrides, e'est pourquoi je les ai mis à part. J'ai aussi établi que le no 45, Corégone du Vivarone, Italie, est un mélange évident de deux formes dont l'une est hybride. La nature de ces hybrides n'apparaît pas dans le tableau, elle devient évidente lorsqu'on calcule la variance, qui exprime la dispersion autour de la moyenne, comme nous le verrons plus loin. On peut aussi évaluer cette dispersion graphiquement, soit en dessinant la courbe de variabilité, comme je l'ai fait dans le travail cité, soit, pour une meilleure lisibilité, en cas de comparaison de plusieurs populations, en portant sur le graphique non plus les fréquences successives, mais les fréquences cumulées. En ordonnées, ces fréquences sont indiquées de 0 à 100\%. L'emploi de l'échelle «probit» (voir Buiss 1937) sur l'axe des ordonnées 
permet d'obtenir, pour une répartition rigoureusement gaussienne, une droite rectiligne oblique. Plus cette droite est oblique, plus la dispersion est grande, naturellement, l'obliquité répondant à l'étalement de la courbe en cloche correspondante. J'admets, en me basant sur mes précédentes études, que cette obliquité est le résultat soit d'une hybridation, soit d'un mélange de deux ou plusieurs formes dont les moyennes sont rapprochées. Si la droite obtenue en cumulant les fréquences successives, au lieu d'être rectiligne, présente des irrégularités vers le haut ou vers le bas, celles-ci sont négligeables, surtout pour les contingents peu nombreux, elles peuvent n'être que des irrégularités fortuites. Si en revanche la droite présente vers le milieu un décrochement pour reprendre ensuite sa direction première, elle correspond à une courbe à deux sommets, elle est alors révélatrice d'un mélange certain de deux formes.

Le graphique (fig. 2) montre la distribution des fréquences de la plupart des populations du tableau $n^{0} 1$. Il est basé sur le nombre des branchiospines du premier arc et montre d'évidence que les populations considérées n'ont pas toutes la même signification.

Les traits épais: $n^{\circ} 1$ (Kilch, Untersee), no $20 p$ (Sandfelchen

Rev. Suisse de Zool., T. 66, 1959.

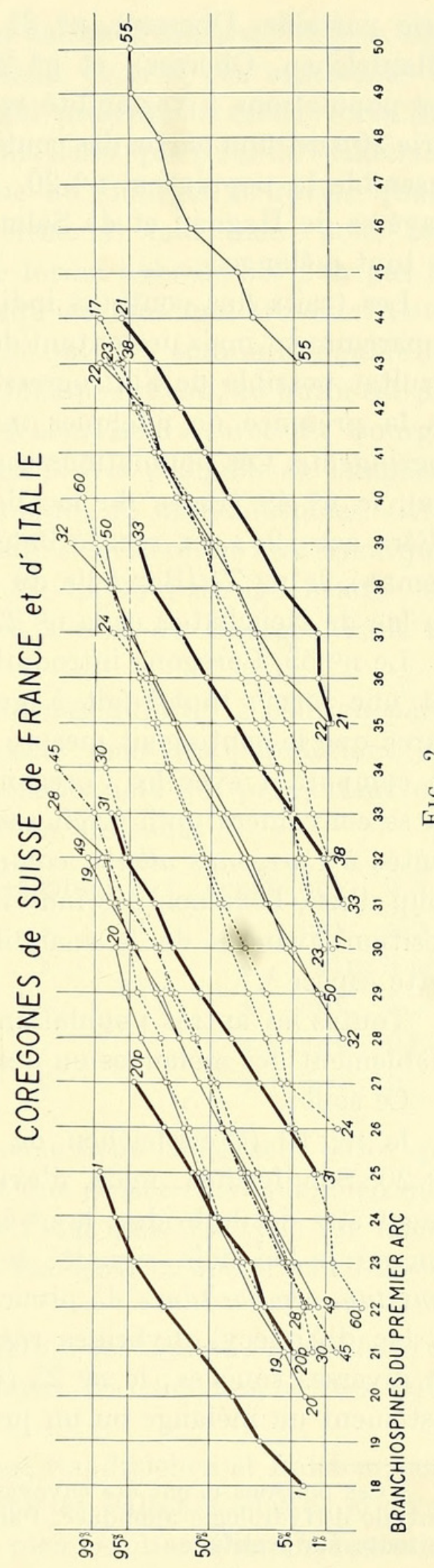


série partielle, Obersee). no 31 (Balchen, lac de Thoune), no 33 (Blaufelchen, Obersee), et $n^{\circ} 21$ (Brienzlig, lac de Brienz), sont des populations à variabilité restreinte et d'ailleurs uniforme. La série $20 p$ est une partie des contingents de Sandfelchen qui forment ensemble la population $n^{0} 20$. Il s'agit de 35 individus des deux frayères de Hagnau et de Salmzach où le type parait encore pur de tout mélange.

Les traits fins continus indiquent des populations assez pures apparemment mais présentant des dispersions un peu plus étendues, résultat possible de l'introgression d'une autre forme, ou, parfois de la présence de quelques individus étrangers égarés dans les contingents. Ces populations sont le no 20 (Sandfelchen de l'Obersee), le no 49 (Palée du lac de Neuchâtel), le no 28 (prétendue «Féra actuelle», en réalité Palée de Neuchâtel, introduite dans le Léman), le no 32 (Bondelle du lac de Bienne), le no 50 (Bondelle du lac de Neuchâtel) et le no 22 (Albock du lac de Thoune).

Le $n^{0} 55$, Corégone introduit dans le lac Chauvet en Auvergne est une forme tout-à-fait à part; la droite est une ligne brisée parce que le contingent mesuré est faible, 22 individus seulement; en comparant avec les Corégones du nord de l'Allemagne (p. 24) on se convaincra qu'il s'agit sans conteste d'une population apparentée à Coregonus albula, comme le prouve d'ailleurs, au premier coup d'œil, l'examen des individus qui présentent presque tous la position avancée de la mandibule inférieure, caractéristique de cette espèce ${ }^{1}$.

Toutes les autres populations — traits pointillés — sont indéniablement des mélanges ou des hybrides.

Ce sont:

le no 19 (Weissfelchen de l'Untersee) presque identique au no 20, Sandfelchen, mais, d'évidence, mélangé d'intrus qui pourraient être des hybrides; le no 45 (Corégone du Vivarone), mélange comme je l'ai déjà signalé; le no 30 (Balchen du lac de Zoug) «mixtum compositum» de pisciculture; les no 24 et 60 (lac Majeur et lac d'Annecy), hybrides résultant de l'apport et du mélange de diverses souches; le no 23 (Gangfisch de l'Untersee) est manifestement un mélange ou un produit hybride, apparemment entre

1 Ces poissons m'ont été envoyés par M. Vivier, directeur de la Station centrale d'Hydrologie appliquée, Paris. Je le prie de trouver ici l'expression de toute ma gratitude. 
le type Weissfelchen et le type pur tel qu'il apparaît encore dans la population naine du lac de Brienz. Je n'ai pas représenté ici le no 42, Gangfisch de l'Obersee, qui présente la même forte dispersion mais une moyenne encore plus basse que celui de l'Untersee.

Le $n^{\circ}$ 17, enfin, Lavaret du lac du Bourget se révèle quasiidentique au Gangfisch de l'Untersee. Il faut bien, pour être logique, l'assimiler, lui aussi à une forme hybridée et non pas au Blaufelchen, comme je l'ai fait jusqu'ici dans mes travaux. Cette rectification supprime une anomalie qui m'a toujours troublé: le fait que le Lavaret fraie en beine, sous peu d'eau, ce qui n'est pas un caractère biologique de Blaufelchen. Le Lavaret du Bourget est donc une forme composite, hybride très probable de Gangfisch et d'une autre forme à nombre moindre de branchiospines. Cette affirmation paraîtra moins surprenante si on se réfère aux remarques de la page 9 et suivantes. Le no 38, Lavaret d'Aiguebelette, est d'évidence une population analogue à celle du Bourget, mais un peu plus nettement Gangfisch.

En définitive, si on exclut les formes sujettes à caution parce qu'hybrides ou mélangées, il subsiste dans les populations des Alpes occidentales, quelques types assez nets qui permettent une répartition en première approximation. Ce sont:

10 le type Kilch, 21 branchiospines env., écarts env. 3,5

20 le type Sandfelchen, 24,5

$3^{\circ}$ le type Balchen, 29

29

$4^{\circ}$ le type Blaufelchen, 36

$5^{\circ}$ le type Gangfisch, 40

60 le type albula,

$\begin{array}{lll}\gg & \text { env. } 4,5 \\ 》 & \text { entre } 5 \text { et } 6 \\ 》 & \text { env. } 7,5 \\ 》 & \text { env. } 9,5 \\ 》 & \text { env. } 13 .\end{array}$

Je continue à appeler le cinquième type Gangfisch, parce que cette forme du lac de Constance est une espèce classique, bien qu'il soit devenu difficile de l'obtenir à l'état pur. Le type est mieux représenté à l'heure actuelle par le Brienzlig et même par l'Albock du lac de Thoune.

Il s'agit, sous le $n^{\circ}$ 6, de la population acclimatée au lac Chauvet, bien distincte avec ses branchiospines très longues et très nombreuses.

Les deuxième et troisième types, Sandfelchen et Balchen, sont reliés par des intermédiaires qui sont les Palées. Celles-ci, si on en juge par leur variabilité, semblent bien résulter d'une hybridation 
ancienne, ou d'une introgression d'une des formes dans l'autre. Il est intéressant de constater que Fatio, en 1890, donnait pour la Palée 22 à 28 (29) branchiospines soit sensiblement la moyenne des Sandfelchen tandis qu'à l'heure actuelle cette moyenne pour la Palée accuse 27 environ. Les Bondelles des mêmes lacs de Neuchâtel et de Bienne se rattachent au Blaufelchen, mais avec leur moyenne de 34 branchiospines elles me paraissent avoir aussi subi une introgression de l'espèce représentée par le Balchen, ce qui est rendu plausible par l'existence de Palées dites de fond qui se reproduisent tardivement, en profondeur au bord du mont et qui se rapprochent en effet des Bondelles (Dottrens et Quartier 1949).

\section{Tableau 2}

Corégones de Bavière et d'Autriche

\begin{tabular}{|c|c|c|c|c|c|c|c|}
\hline d'ordre & $\begin{array}{l}\text { Population } \\
\text { (non usuel) }\end{array}$ & Lac & $\begin{array}{l}\text { Nom- } \\
\text { bre } \\
\text { d'indi- } \\
\text { vidus }\end{array}$ & $\begin{array}{c}\text { Nombre de } \\
\text { branchiospines }\end{array}$ & Ecarts & $\begin{array}{l}\text { Lon- } \\
\text { gueur } \\
\text { moy. } \\
\text { en cm }\end{array}$ & $\begin{array}{c}\text { Epoque } \\
\text { de capture }\end{array}$ \\
\hline \multicolumn{8}{|c|}{$1^{\mathrm{er}}$ type: aucune } \\
\hline \multicolumn{8}{|c|}{$2^{e}$ type (Sandfelchen) } \\
\hline 48 & Renke & Chiemsee & 183 & $25,7 \pm 0,15$ & $5,2 \pm 0,05$ & 31 & août \\
\hline \multicolumn{8}{|c|}{ 3e type (Balchen) } \\
\hline $\begin{array}{l}10 \\
62\end{array}$ & $\begin{array}{l}\text { Reinanke } \\
\text { Renke }\end{array}$ & $\begin{array}{l}\text { Attersee } \\
\text { Walchensee }\end{array}$ & $\begin{array}{l}90 \\
27\end{array}$ & $\begin{array}{l}29,0 \pm 0,26 \\
29,8 \pm 0,49\end{array}$ & $6,5 \pm 0,09$ & $\begin{array}{l}36 \\
39\end{array}$ & $\begin{array}{l}\text { mi-octobre } \\
\text { juin-juillet }\end{array}$ \\
\hline \multicolumn{8}{|c|}{$4^{\mathrm{e}}$ type (Blaufelchen) } \\
\hline $\begin{array}{r}12 \\
8 \\
37 \\
44 \mathrm{~A}\end{array}$ & $\begin{array}{l}\text { Renke } \\
\text { Kröpfling } \\
\text { Renke } \\
\text { Reinanke }\end{array}$ & $\begin{array}{l}\text { Ammersee } \\
\text { Attersee } \\
\text { Tegernsee } \\
\text { Hallstättersee }\end{array}$ & $\begin{array}{r}41 \\
54 \\
163 \\
20\end{array}$ & $\begin{array}{l}35,4 \pm 0,28 \\
35,8 \pm 0,32 \\
37,4 \pm 0,17 \\
37,7 \pm 0,34\end{array}$ & $\begin{array}{l}7,9 \pm 0,07 \\
8,2 \pm 0,12 \\
8,6 \pm 0,08 \\
7,6 \pm 0,14\end{array}$ & $\begin{array}{l}36 \\
31 \\
46 \\
31\end{array}$ & $\begin{array}{l}\text { juin } \\
\text { mi-octobre } \\
\text { juin } \\
\text { novembre }\end{array}$ \\
\hline 11 & Renke & Würmsee & 133 & $38,5 \pm 0,22$ & $8,8 \pm 0,06$ & 35 & juin \\
\hline \multicolumn{8}{|c|}{$5^{\text {e }}$ type (Gangfisch) } \\
\hline $\begin{array}{l}57 \\
44 \mathrm{~B}\end{array}$ & $\begin{array}{l}\text { Renke } \\
\text { Reinanke }\end{array}$ & $\begin{array}{l}\text { Riegsee } \\
\text { Hallstättersee }\end{array}$ & $\begin{array}{l}51 \\
65\end{array}$ & $\begin{array}{l}42,0 \pm 0,28 \\
42,9 \pm 0,27\end{array}$ & $\begin{array}{l}9,5 \pm 0,13 \\
9,8 \pm 0,11\end{array}$ & $\begin{array}{l}39 \\
32\end{array}$ & $\begin{array}{l}\text { juin } \\
\text { novembre }\end{array}$ \\
\hline
\end{tabular}


On remarquera que cette répartition se superposerait parfaitement à celle de WAGLER, qui reconnaît quatre espèces, n'était le type Balchen qui lui, correspond au type primigenius de SternMANN.

En sorte que je suis amené à penser que Sternmann a bien pressenti l'existence de ce troisième type, mais qu'il l'aurait confondu avec des populations hybrides ou mélangées qui accusent un nombre moyen de branchiospines comparable.

WAgler (1950) a publié des listes des espèces présentes dans les lacs de Bavière et d'Autriche, d'où il résulterait que deux lacs, le Chiemsee et l'Ammersee, hébergeraient ses quatre espèces. Il en existerait trois dans le Würmsee, le Traunsee, le Walchensee, le Tegernsee et l'Attersee. Dans le Chiemsee, par exemple, où le sondage que j'ai fait n'a révélé l'existence que d'une seule forme, manifestement apparentée au Sandfelchen (no 48), il affirme l'existence du Gangfisch et du Kilch, qui vivraient côte à côte, auraient le mêm e faible nom bre de branchios pines ce qui aurait empêché les auteurs de les reconnaître: «Kilch und Gangfisch leben im Chiemsee nebeneinander, nur hat die ähnliche, niedere Reusendohrnzahl bei den Fischen die saubere Trennung bisher verhindert». On admettra qu'il est difficile de suivre cet auteur quand ses déterminations sont à ce point subjectives. Pour le Würmsee, grâce à l'obligeante assistance du Dr REHBRonn, j'ai pu faire mieux qu'un sondage; j'ai pu disposer de plusieurs contingents successifs prélevés dans le lac à des époques différentes. (Dans le tableau, ne figurent que les individus que j’ai mesuré moimême sur place, à l'exclusion de tous ceux qui ont été examinés pour moi à Starnberg et qui donnent le même résultat.) Dans aucun de ces contingents je n'ai pu déceler la présence des trois espèces que Wagler pensait avoir reconnues. Ces contingents correspondent toujours au même ensemble à grande variabilité, manifestement hybridé ( $n^{\circ} 11$ du tableau).

Au Tegernsee (no 37 ), j’ai déjà noté que les individus pêchés au fond étaient pratiquement identiques à ceux de pleine eau. Pour le Walchensee où Wagler note trois espèces, j’ai obtenu du Dr Schinder que j'ai plaisir à remercier ici, deux petits échantillons, 27 individus en tout, qui donnent l'impression d'une population intimément mélangée, hybride probablement des populations qu'on y a certainement introduites. Le tableau comporte encore le 
résultat d'un sondage effectué en période de fraie au lac de Hallstatt, grâce à l'aide bienveillante du $\mathrm{D}^{\mathrm{r}}$ Einsele. Les Reinanken pêchés en un ou deux jours en novembre appartenaient d'évidence à deux formes différentes que j'ai séparées après coup, a r t i f i c i e lle ment. J'avais noté sur place, par chance, les femelles qui n'étaient pas mûres; presque toutes accusèrent un nombre de branchiospines relativement faible et montrèrent des branchiospines relativement courtes. Séparées du lot principal avec les mâles qui d'évidence présentaient des caractères analogues, elles ont constitué le groupe «inférieus" no $44 \mathrm{~A}$. Pour plusieurs de ces individus, j'avais noté au passage des indications telles que 《museau pointu», «nageoires très enfumées », «très noircissant » qui me suggéraient l'idée qu'il pouvait s'agir de vrais Blaufelchen. Il est probable qu'en fixant son attention sur ces caractères en partie fugaces, on pourrait d'une manière satisfaisante sélectionner sur place les deux formes. Tout me porte à penser que la forme $44 \mathrm{~B}$, autochtone sans doute, est un Gangfisch et que l'autre, $44 \mathrm{~A}$, a été importée (du Mondsee ?); celle-ci, qui n'est pas mûre en novembre, est un Blaufelchen. Or WAgLer, n'indique pour le lac de Hallstatt que le seul Blaufelchen auquel il attribue une moyenne du nombre de branchiospines égale à 41,9. Je trouve pour l'ensemble du contingent que j'ai mesuré, 85 individus dont $65 \mathrm{du}$ groupe «supérieur»: 41,7 $\pm 0,33$.

Le tableau no 2 ne fournit qu'un aperçu des Corégones de Bavière et d'Autriche, il correspond à une première reconnaissance, mais suffisante pour montrer que le système manifestement artificiel de Wagler pour ces régions doit être entièrement revu et objectivement corrigé. Il est en effet possible qu'il existe des Kilche au Chiemsee et dans l'Ammersee, mais je n'ai pas eu l'occasion de les rencontrer.

Le graphique (fig. 3) correspond au tableau 2.

Parmi les populations relativement pures — traits pleins le $n^{0} 48$, Renke du Chiemsee, doit être rapporté au Sandfelchen, les no 8 (Kröpfling Attersee) 12 (Renke Ammersee) et 37 (Renke Tegernsee) doivent être considérés comme des Blaufelchen encore suffisamment caractéristiques; de même que $44 \mathrm{~A}$ produit de triage.

Les nos 57 (Renke du Riegsee) et $44 \mathrm{~B}$ ne peuvent être que des Gang fisch malgré leur taille, considérable surtout chez les Renken 
Tableau 3

Corégones du Nord: Holstein (Allemagne), Jutland (Danemark), Jämtland (Suède)

\begin{tabular}{|c|c|c|c|c|c|c|c|}
\hline d'ordre & $\begin{array}{l}\text { Population } \\
\text { (non usuel) }\end{array}$ & Lac & $\begin{array}{l}\text { Nom- } \\
\text { bre } \\
\text { d'indi- } \\
\text { vidus }\end{array}$ & $\begin{array}{l}\text { Nombre de } \\
\text { branchio- } \\
\text { spines }\end{array}$ & Écarts & $\begin{array}{l}\text { Lon- } \\
\text { gueur } \\
\text { moy. } \\
\text { en cm }\end{array}$ & $\begin{array}{c}\text { Epoque } \\
\text { de capture }\end{array}$ \\
\hline \multicolumn{8}{|c|}{$1^{\mathrm{er}}$ type: Kilch } \\
\hline $\begin{array}{r}26 \\
47 \\
2\end{array}$ & $\underset{y}{\text { Storsik }}$ & $\begin{array}{l}\text { Näckten (S.) } \\
\text { Ismundsjön } \\
\text { Storsjön }\end{array}$ & $\begin{array}{c}55 \\
13 \\
61\end{array}$ & $\begin{array}{l}20,7 \pm 0,22 \\
21,9 \pm 0,47 \\
22,0 \pm 0,22\end{array}$ & $\begin{array}{l}3,1 \pm 0,06 \\
3,4 \pm 0,08 \\
3,8 \pm 0,08\end{array}$ & $\begin{array}{c}(36) \\
(40) \\
39\end{array}$ & $\begin{array}{l}\text { déc. en fraie } \\
\text { nov. en fraie }\end{array}$ \\
\hline \multicolumn{8}{|c|}{$2^{\text {e }}$ type: Sandfelchen } \\
\hline $\begin{array}{l}56 \\
27\end{array}$ & $\begin{array}{l}\text { Grosse Maräne } \\
\text { Älvsik }\end{array}$ & $\begin{array}{l}\text { Selentersee (A) } \\
\text { Storsjön (S.) }\end{array}$ & $\begin{array}{l}118 \\
101\end{array}$ & $\begin{array}{l}24,2 \pm 0,16 \\
26,9 \pm 0,31\end{array}$ & $\begin{array}{l}4,5 \pm 0,05 \\
4,8 \pm 0,10\end{array}$ & $\begin{array}{l}42 \\
26\end{array}$ & $\begin{array}{l}\text { août } \\
\text { octobre en fraie }\end{array}$ \\
\hline \multicolumn{8}{|c|}{$3^{\mathrm{e}}$ type: Balchen } \\
\hline $\begin{array}{r}15 \\
13 \\
9\end{array}$ & $\begin{array}{l}\text { Storsik } \\
\text { Älvsik } \\
\text { Schnäpel }\end{array}$ & $\begin{array}{l}\text { Landösjön (S.) } \\
\text { Alsensjön } \\
\text { Ringköbbing (D.) }\end{array}$ & $\begin{array}{l}24 \\
94 \\
86\end{array}$ & $\begin{array}{l}28,5 \pm 0,30 \\
28,8 \pm 0,25 \\
29,1 \pm 0,19\end{array}$ & $\begin{array}{l}5,1 \pm 0,15 \\
5,3 \pm 0,09 \\
5,8 \pm 0,07\end{array}$ & $\begin{array}{c}36 \\
(25) \\
(34)\end{array}$ & $\begin{array}{l}\text { nov: en fraie } \\
\text { fin nov. en fraie } \\
\text { " }\end{array}$ \\
\hline 46 & Blåsik & $\begin{array}{l}\text { Storsjön (S.) } \\
\text { (Änge) }\end{array}$ & 186 & $30,4 \pm 0,29$ & $5,9 \pm 0,10$ & $(22)$ & novembre \\
\hline
\end{tabular}

$4^{\text {e }}$ type: Blaufelchen

\begin{tabular}{|c|c|c|c|c|c|c|c|}
\hline $\begin{array}{l}34 \\
25 \\
51 \\
52\end{array}$ & $\begin{array}{l}\text { Storsik } \\
\text { „ } \\
\text { Blâsik } \\
\text { Älvsik }\end{array}$ & $\begin{array}{l}\text { Skällbrägden } \\
\text { Fullsjön } \\
\text { Bolesjön } \\
\text { Bolesjön }\end{array}$ & $\begin{array}{l}74 \\
22 \\
20 \\
44\end{array}$ & $\begin{array}{l}36,2 \pm 0,43 \\
36,2 \pm 0,37 \\
36,3 \pm 0,38 \\
36,3 \pm 0,34\end{array}$ & $\begin{array}{l}6,0 \pm 0,12 \\
7,9 \pm 0,17 \\
7,5 \pm 0,16 \\
7,2 \pm 0,12\end{array}$ & $\begin{array}{l}(28) \\
(36) \\
24 \\
23\end{array}$ & $\begin{array}{l}\text { janv. en fraie } \\
\text { début déc. en fraie } \\
\text { fin nov. en fraie } \\
\text { mi-déc. en fraie }\end{array}$ \\
\hline $\begin{array}{l}36 \\
35 \\
14\end{array}$ & $\begin{array}{l}\text { Grråsik } \\
\text { Älvsik } \\
\text { Småsik }\end{array}$ & $\begin{array}{l}\text { Revsundsjön } \\
\text { Gröttingen } \\
\text { Sundsjön }\end{array}$ & $\begin{array}{l}75 \\
49 \\
96\end{array}$ & $\begin{array}{l}36,6 \pm 0,22 \\
36,8 \pm 0,29 \\
37,1 \pm 0,21\end{array}$ & $\begin{array}{l}7,3 \pm 0,09 \\
8,5 \pm 0,13 \\
7,4 \pm 0,06\end{array}$ & $\begin{array}{l}31 \\
29 \\
(24)\end{array}$ & $\begin{array}{l}\text { fin nov. en fraie } \\
\text { fin nov. en fraie } \\
\text { mi-déc. en fraie }\end{array}$ \\
\hline
\end{tabular}

$5^{\text {e }}$ type: Gangfisch

\begin{tabular}{|c|c|c|c|c|c|c|c|}
\hline $\begin{array}{l}39 \\
41\end{array}$ & $\begin{array}{l}\text { Småsik } \\
\text { Älvsik }\end{array}$ & $\begin{array}{l}\text { Näckten } \\
\text { Bodsjön }\end{array}$ & $\begin{array}{l}50 \\
30\end{array}$ & $\begin{array}{l}39,3 \pm 0,23 \\
40,0 \pm 0,44\end{array}$ & $\begin{array}{l}9,2 \pm 0,13 \\
8,4 \pm 0,17\end{array}$ & $\begin{array}{c}(16) \\
30\end{array}$ & $\begin{array}{l}\text { janv. en fraie } \\
\text { fin nov. en fraie }\end{array}$ \\
\hline 43 & Smärling & Locknesjön & 99 & $41,9 \pm 0,25$ & $9,9 \pm 0,09$ & 22 & janv. en fraie \\
\hline
\end{tabular}

$6^{\text {e }}$ type: Coregonus albula

\begin{tabular}{l|l|l|l|l|l|l|l}
\hline 54 & Kleine Märane & Plönersee (A.) & 97 & $42,6 \pm 0,23$ & $13,3 \pm 0,10$ & 21 & août \\
58 & Dieksee & 100 & $42,6 \pm 0,19$ & $13,5 \pm 0,11$ & 21 & " \\
59 & Pönitzersee & 100 & $43,0 \pm 0,17$ & $13,8 \pm 0,10$ & 21 & " \\
53 & Schalsee & 21 & $43,2 \pm 0,34$ & $13,9 \pm 0,27$ & 23 & " \\
\hline
\end{tabular}

(Entre parenthèses, mesures prises sur individus isolés.) 

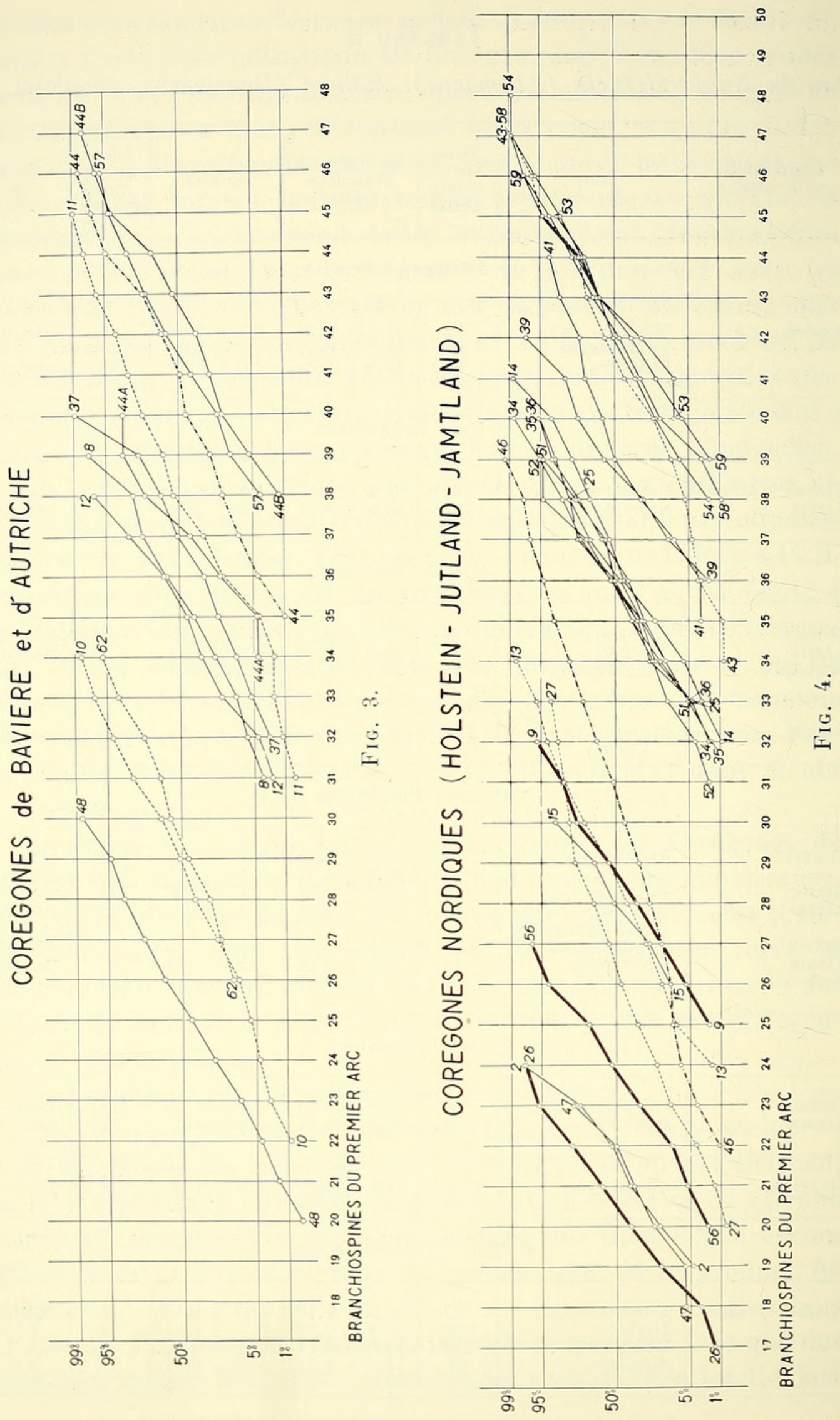
du Riegsee. Les trois autres populations - traits pointillés sont hybridées, ce sont celle du Walchensee (no 62) et la Reinanke de l'Attersee (no 10) qui par leurs moyennes répondent au Balchen, puis le no 11 (Würmsee) qui me paraît un hybride manifeste de Gangfisch et de Blaufelchen. Le $n^{0} 44$, combinaison de $44 \mathrm{~A}$ et $44 \mathrm{~B}$, montre l'aspect de la courbe dans le cas du mélange de deux formes différentes.

Dans l'ensemble, il me paraît remarquable qu'aucune des populations que j'ai examinées en Bavière et en Autriche ne présente la dispersion restreinte des formes vraiment pures. Les manipulations de l'homme dans ces deux régions ont été telles qu'il n'y subsiste vraisemblablement plus de formes intactes et typiques.

Je suis redevable de la possibilité d'avoir étudié les Corégones du Nord à la bienveillante assistance du Professeur Thienemann, Plön, du Dr Marre, Kiel, du Dr Curry-Lindahl, Stockolm, et du Dr Svärdson, Drottningholm. Qu'ils veuillent bien trouver ici l'expression de toute ma gratitude.

On remarquera d'emblée que le classement des populations nordiques selon les types reconnus dans les lacs alpins ne semble offrir aucune difficulté.

Le graphique (fig. 4) présente la dispersion pour chacune des populations du tableau 3. On y retrouve les mêmes caractéristiques que dans les Corégones des Alpes, et même des populations à faible dispersion dont la courbe - en traits épais - se superpose presque exactement à celle des populations alpines typiques correspondantes.

Dans le premier groupe, type Kilch, le Storsik du Näckten (no 26) paraît le plus pur tandis que le Storsik de l'Ismundsjön ( $n^{0} 47$ ), mais qui n'est représenté que par 13 individus, et surtout celui du Storsjön ( $n^{0} 2$ ) pourraient bien comporter des individus du type Sandfelchen ou avoir subi une certaine introgression.

Le deuxième type n'est représenté que par la Grande Marène du Selentersee, que Thienemann a distinguée naguère sous le nom de Coregonus holsatus. Il ne fait pas de doute que cette Marène du Holstein est un Sandfelchen typique. Le no 27, tout au contraire, est certainement une population hybridée. Le contingent a été pêché sur les frayères d'Ytterån, il paraît parfaitement homogène, l'hypothèse d'un éventuel mélange doit donc être rejetée. Le Blåsik du même Storsjön figure à part au bas du troisième groupe. 
Le graphique (fig. 4) montre que les contingents ( $n^{\circ} 46$ ) capturés eux aussi sur un seul emplacement de fraie, sous le barrage d'Änge, appartiennent à une population composite. Il s'agit d'un mélange indéniable de deux formes. La moins abondante ne se distingue guère à première vue que par un nombre moindre de branchiospines. J'ai trié, après coup et a $\mathrm{t}$ ifici e $1 \mathrm{l}$ e m e $\mathrm{nt}$ les individus formant la partie inférieure de la courbe bimodale, et obtenu ainsi le «groupe inférieur d'Änge», les individus restants formant le groupe supérieur. Voici le résultat de ce tri:

\begin{tabular}{|c|c|c|c|c|c|}
\hline Groupe & $\begin{array}{c}\text { Nombre } \\
\text { d'indi- } \\
\text { vidus }\end{array}$ & $\begin{array}{c}\text { Nombre } \\
\text { de bran- } \\
\text { chiospines }\end{array}$ & Ecarts & $\begin{array}{c}\text { Nombre } \\
\text { d’écailles } \\
\text { latérales }\end{array}$ & $\begin{array}{c}\text { Longueur } \\
\text { moyenne } \\
\text { en cm. }\end{array}$ \\
\hline Inférieur . . & 35 & $24,9 \pm 0,30$ & $3,9 \pm 0,08$ & 96 & 24 \\
Supérieur. . & 140 & $31,8 \pm 0,24$ & $6,3 \pm 0,09$ & 93 & 21 \\
\hline
\end{tabular}

On voit que le groupe inférieur peut être interprété comme appartenant au type Sandfelchen, il se distingue par une taille moyenne supérieure à celle de l'autre groupe. Celui-ci, vu sa variabilité ne peut guère être considéré que comme un hybride. Le barrage d'Änge fonctionnant comme une trappe pour les Corégones qui arrivent sur leurs frayères, on peut se demander dans quelle mesure cette intervention humaine a favorisé ou même provoqué cette hybridation. On pourra comparer cette population composite à celle du lac de Vivarone (Dottrens 1955) où l'intervention humaine a mélangé une forme hybridée avec une forme importée séparément.

Le Storsjön, qui est le plus vaste lac du Jämtland, héberge quatre espèces différentes de Corégones. Malgré le dévouement si attentif de M. Lundgren, Fiskerikonsultent à Östersund, je n'ai pu mesurer que le Storsik capturé sur les frayères des rapides de Kwissle, en novembre, l'Älvsik d'Ytterån et le Blåsik d'Änge qui frayent également en rivière à peu près à la même époque. Je n'ai pas pu obtenir de Blåsik, ni de Planktonsik, qui frayent au lac en décembre-janvier d'après LundGren. Je ne peux donc pas juger personnellement de la nature de ces formes qui sont peut-être pures (d'après la tabelle, p. 154, de Svärdson (1953), le planktonsik avec br. $=37,6$ me paraît une population pure, tandis que le Blåsik avec br. = 32.8 serait hybridé). Je suis surpris du mélange 
évident et de l'hybridation presque certaine des populations pêchées à Ytterån et à Änge. Mème le Storsik pêché dans les courants de de Kwissle pourrait bien ètre mélangé, l'inflexion de la droite entre 25 et $75 \%$ étant considérable pour un contingent de 60 individus.

Cette impureté des populations se retrouve dans l'Alsensjön, qui n'est séparé du Storsjön que par le courant d'Ytterån. Le no 13, Älvsik de l'Alsensjön, provient de la frayère de Vaplan, au débouché dans ce lac du courant provenant du Näldsjön. Cette population, bien que se rapportant apparemment au type Balchen, présente une dispersion exagérée. Quant au no 15, Storsik du Landösjön, il ne s'agit que d'un contingent restreint et par conséquent discutable. Il paraît typique du groupe Balchen, mais SvÄrdson (communication personnelle) me fait remarquer qu'il doit s'agir en réalité d'un mélange résultant de l'immersion dans le lac voisin, Rönnösjön, d'une forme à 25-26 branchiospines qui s'est ensuite propagée pour rejoindre la population existant déjà dans le Landösjön. Il s'agit donc d'un cas qu'il faudrait élucider (voir p. 52).

En définitive, je n'ai rencontré, du type Balchen dans le Nord, que le seul Schnäpel du Ringköbbingfjord, no 9, qui soit vraiment typique. Il s'agit d'un contingent que j'ai reçu d'une maison de commerce d'Esbjerg, Jutland, grâce à l'entremise aimable de M. C. J. Rasmussen, Charlottenlund. Le Schnäpel présente l'homogénéité parfaite et la dispersion restreinte des populations pures. Je le considérerais volontiers comme le type même du troisième groupe, il est d'ailleurs indiscernable du Balchen de Thoune pour la moyenne du nombre des branchiospines: $29,1 \pm 0,18$ ou 0,19. Svärdson (1957) donne un relevé de diverses populations des côtes de la Baltique parmi lesquelles un certain nombre présentent une moyenne voisine de celle du Schnäpel. Il les considère (communication personnelle) comme le résultat de la fusion complète des formes à 25-27 branchiospines et 29-31 branchiospines qui sont nettement séparées ailleurs. Je reviendrai sur cette importante divergence d'interprétation (p. 36).

Par contraste avec le troisième groupe, le quatrième, dans le Jämtland est d'une homogénéité qui ne laisse rien à désirer. Dans cette province, en tout cas, le quatrième type, qu'il s'appelle Blåsik, Gråsik, Älvsik, Småsik voire même Storsik, est toujours parfaitement reconnaissable et accuse très nettement 36 ou 37 branchiospines en moyenne. Les nos 51 et 52, Blåsik et Älvsik 
du Bolesjön ne diffèrent guère que par l'emplacement de leurs frayères. En effet, on entend sous le nom de Hållstagruppen un chapelet de petits lacs dont l'émissaire aboutit au Revsundsjön. Le Bolesjön est le lac médian de la série. La frayère de Ballstaon, où se réunissent les Älvsik est sur la rivière en amont, celle où s'assemblent les Blåsik est à l'aval.

Je n'ai obtenu aucune population dans le Jämtland qui ait une moyenne de 31 à 34 branchiospines caractéristique de Coregonus lavaretus d'après Svärdson. Mais cet auteur indique lui-même l'existence en Scandinavie de populations à 36-37 branchiospines dont la variabilité n'est pas supérieure à celle des populations à 31-34 branchiospines, au contraire, et qui répondraient parfaitement au type tel que je l'admets ici. L'examen plus attentif de ce quatrième groupe montrera sans doute les raisons de la fluctuation de ces moyennes selon les populations.

Le cinquième groupe, type Gangfisch, n'est guère représenté dans les contingents que j'ai pu mesurer dans le Jämtland, il paraît manquer totalement en Allemagne du Nord. Seul, le no 29, Småsik du Näckten, qui fraie au bord du lac sauf erreur, paraît en être un représentant authentique. Le no 41, Älvsik du Bodsjön, dont la frayère se situe dans des rapides et qui se reproduit en novembre paraît moins pur, mais le petit contingent de 30 individus que j'ai eu entre les mains est trop faible pour permettre une affirmation. La moitié au moins des individus capturés en fin de fraie se sont révélés de véritables pillards de leurs propres œufs. Dans le cas particulier, en tout cas, la notion d'anorexie de reproduction m'a paru presque un mythe!

Quant au no 43, Smärling du Locknesjön, qui fraie au lac fin décembre-janvier, il est indubitablement une forme composite, un hybride, car les courbes de variabilité des divers caractères sont régulières. Or, ce ne peut être qu'un hybride de Gangfisch et de petite Marène. En effet, la plus grande partie de la courbe des fréquences des branchiospines se superpose à celle qui est typique de Coregonus albula. Mais on ne trouve qu'un nombre restreint d'individus présentant une nette proéminence de la mandibule inférieure, la plupart ayant une bouche terminale ou même inférieure. D'autre part, le nombre d'écarts correspondant à la plus grande branchiospine, presque 10 en moyenne, est intermédiaire entre les moyennes du cinquième type et celles des albula. Si 
l'interprétation est juste, ce Smärling serait la preuve que Coregonus albula ne se singularise en aucune façon et ne s'oppose par conséquent pas à l'ensemble des autres Corégones. Le sixième type est représenté par les populations de $C$. albula du Holstein. Ces populations forment un groupe compact, un ensemble uniforme. Pourtant, l'examen des courbes de variation montre que les lacs, comme le Plönersee, qui hébergent d'autres Corégones ont des populations d'albula plus fluctuantes. Introgression vraisemblable et indice de plus que $C$. albula n'est qu'une espèce parmi d'autres du même genre.

Avant de passer aux Corégones de Grande Bretagne, il n'est peut-être pas superflu de confronter les résultats de mes mesures en Suède avec ceux de Svärdson (1957) quand ils sont comparables. Dans le tableau no 4, je reprends mes propres données en les présentant dans l'ordre géographique suivi par cet auteur:

TABLEAU 4

Corégones du système de la rivière Indalsälven

Svärdson Moi-même

Storsjön (Kwissleströmmenen) . . 49 ind. $22,1 \quad 61$ ind. $22,1 \pm 0,22$

Näckten . . . . . . . . 120 ind. $38,9 \quad 50$ ind. $39,3 \pm 0,23$

Corégones du système de la rivière Ljungan

Locknesjön . . . . . gr. sup. env. 130 ind. $(41,5) \quad 99$ ind. $41,9 \pm 0,25$

Revsundsjön . . . . . . 167 ind. $36,7 \quad 75$ ind. $36,6 \pm 0,22$

Sundsjön . . . . . . . . 50 ind. $37,2 \quad 96$ ind. $37,1 \pm 0,21$

La correspondance est tout-à-fait satisfaisante.

Je dois mon matériel de Grande-Bretagne à l'aide cordiale de plusieurs collègues à qui va toute ma reconnaissance: $\mathrm{D}^{\mathrm{r}} \mathrm{E}$. TrEwavas, British Museum, Dr W. Frost, Laboratoire de Windermere, Dr H. D. Slack, Glasgow, Dr J. W. Jones, Liverpool, et le Professeur Gresson qui m'a procuré les Corégones d'Irlande du Nord.

Le tableau 5 est difficile à interpréter. Il est indispensable pour le comprendre de considérer la dispersion pour chaque population, telle qu'elle ressort du graphique (fig. 5).

La population la plus pure est apparemment le Pollan du Lough Neagh ( $n^{\circ} 5$ ) dont le nombre de branchiospines est faible pour un Gangfisch, mais la longueur relative des branchiospines est caractérisitique (écarts: 9,3). 
Tableau 5

Corégones de Grande-Bretagne et d'Irlande

\begin{tabular}{|c|c|c|c|c|c|c|c|}
\hline $\begin{array}{l}\mathrm{N}^{\circ} \\
\text { d'ordre }\end{array}$ & Population & Lac & $\begin{array}{l}\text { Nom- } \\
\text { bre } \\
\text { d’indi- } \\
\text { vidus }\end{array}$ & $\begin{array}{l}\text { Nombre de } \\
\text { branchio- } \\
\text { spines }\end{array}$ & Ecarts & $\begin{array}{l}\text { Long. } \\
\text { en } \mathrm{cm}\end{array}$ & $\begin{array}{c}\text { Epoque } \\
\text { de capture }\end{array}$ \\
\hline \multicolumn{8}{|c|}{$1^{\mathrm{es}}$ type: Kilch, aucune } \\
\hline \multicolumn{8}{|c|}{$2^{\text {e }}$ type: Sandfelchen, aucune } \\
\hline \multicolumn{8}{|c|}{ 3e type: Balchen, aucune } \\
\hline \multicolumn{8}{|c|}{$4^{\mathrm{e}}$ type: Blaufelchen } \\
\hline $6 \stackrel{3}{6}{ }_{7}^{\mathrm{B}}$ & $\begin{array}{l}\text { Powan } \\
\text { Skelly } \\
\text { Gwyniad }\end{array}$ & $\begin{array}{l}\text { Loch Lomond } \\
\text { Ullswater } \\
\text { Bala (Tegid) }\end{array}$ & $\begin{array}{r}387 \\
59 \\
23\end{array}$ & $\begin{array}{l}33,9 \pm 0,08 \\
37,1 \pm 0,21 \\
37,3 \pm 0,40\end{array}$ & $\begin{array}{l}6,7 \pm 0,04 \\
8,2 \pm 0,12 \\
8,1 \pm 0,2\end{array}$ & $\begin{array}{l}28 \\
38 \\
(24)\end{array}$ & $\begin{array}{l}\text { diverses époques } \\
\text { printemps } \\
\text { de collections }\end{array}$ \\
\hline $6 \mathrm{~A}$ & Skelly & Haweswater & 35 & $37,7 \pm 0,30$ & $9,0 \pm 0,12$ & 32 & printemps \\
\hline \multicolumn{8}{|c|}{$5^{\text {e }}$ type: Gangfisch } \\
\hline $\begin{array}{r}5 \\
40\end{array}$ & $\begin{array}{l}\text { Pollan } \\
\text { Gwyniad }\end{array}$ & $\begin{array}{l}\text { Long Neagh } \\
\text { Bala (Tegid) }\end{array}$ & $\begin{array}{l}98 \\
52\end{array}$ & $\begin{array}{l}38,2 \pm 0,15 \\
39,5 \pm 0,23\end{array}$ & $\begin{array}{l}9,3 \pm 0,08 \\
8,1 \pm 0,13\end{array}$ & $\begin{array}{l}26 \\
(26)\end{array}$ & $\begin{array}{l}\text { printemps } \\
\text { novembre }\end{array}$ \\
\hline \multicolumn{8}{|c|}{$6^{\text {e }}$ type: albula } \\
\hline 4 & Pollan & Longh Erne & 94 & $41,8 \pm 0,20$ & $12,4 \pm 0,11$ & $(26)$ & printemps \\
\hline
\end{tabular}

(Entre parenthèses, mesures prises sur individus fixés.)

FIG. 5.

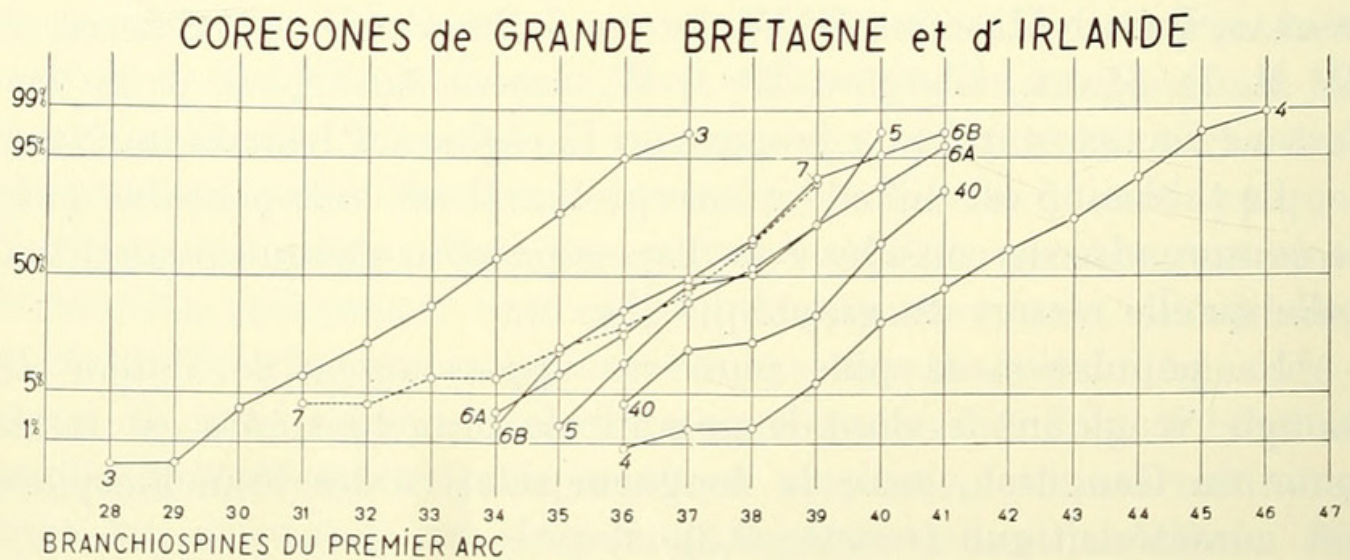


Le Powan du Loch Lomond, $n^{\circ} 3$, est une population homogène placée pour le nombre des branchiospines à la limite inférieure du type Blaufelchen. Dans ma note au Congrès de Londres (Dotтrens 1958), j'avais admis, pour cette population une certaine introgression, qui est possible mais non évidente. Les Skellies, $6 \mathrm{~A}$ et $6 \mathrm{~B}$, sont certainement apparentés mais celui de Haweswater, $6 \mathrm{~A}$, avec le décrochement de la droite de dispersion aux environs de $50 \%$ donne l'impression d'un mélange. Le nombre d'individus considérés n'est pas suffisant pour être affirmatif. Il se pourrait qu'il s'agisse d'un Blaufelchen, comme le Skelly de Ullswater, mais ayant subi une notable introgression. La population du lac Bala (Llyn Tegid) est d'évidence composite. Les exemplaires de collections de Londres et de Liverpool qui constituent la série no 7 se rattachent sans aucun doute au type Blaufelchen mais la variabilité du nombre des branchiospines présente une anomalie troublante. Ceux de la population no 40 pêchés en novembre 1955 par le $\mathrm{D}^{\mathrm{r}} \mathrm{J}$. W. Jones sont incontestablement du type Gangfisch. Ils ont seulement des branchiospines très souvent lésées et le nombre moyen des écarts, 8,1 seulement, s'en trouve accidentellement abaissé.

Le Pollan du Lough Erne enfin, $n^{0} 4$, qui est incontestablement une petite Marène, C. albula, n'est pas une population pure. Elle a subi une introgression d'une forme analogue à celle du Lough Neagh. En effet, si la longueur relative des branchiospines est typiquement albula, la moyenne du nombre des branchiospines se rapproche de celle des Gangfisch, tandis que la dispersion est celle d'une population plus ou moins hybridée. Il est remarquable qu'il n'y ait, dans les eaux intérieures britanniques aucune population des trois premiers types.

\section{Tableaux RÉCapitulatifs}

Les quelque 60 populations que j'ai étudiées biométriquement peuvent se classer, quant à la variabilité du nombre des branchiospines, mais d'une manière quelque peu arbitraire, en trois catégories: populations pures ou relativement pures, populations relativement peu modifiées par mélanges, introgression ou hybridations, populations nettement altérées. 
Je me suis basé, pour faire ce triage sur la variance exprimée par la formule $\mathrm{V}=\frac{\Sigma(\mathrm{xi}-\overline{\mathrm{x}})^{2}}{\mathrm{n}}$, qui est le carré de l'écart-type ou standard deviation. Je fournis également dans chaque cas le


Laмотте (1948) permettrait mieux de comparer la dispersion des diverses distributions puisqu'il est la mesure de la dispersion relative. On observera que cette dispersion relative est nettement supérieure dans les populations à branchiospines nombreuses, ce qui va de soit si la variance est constante d'un type extrême à l'autre, du moins apparemment, chez les populations pures.

$\mathrm{Si}$ on considère les seules populations relativement pures du tableau no 6 , on ne peut être que frappé de la clarté de leur classement en 6 types distincts. Tout au plus peut-on remarquer l'anomalie présentée par le Skelly de Haweswater pour le nombre d'écarts qui correspond au type Gangfisch alors que le nombre de branchiospines peut encore être considéré comme à la limite au moins des Blaufelchen. J'ai déjà dit qu'il pourrait s'agir d'introgression, mais un mélange n'est pas exclu. Le nombre des individus pris en considération étant relativement petit, il faudrait reprendre les populations de Skellies comme du reste le Gwyniad du lac Bala pour déterminer leur statut exact. On remarquera aussi la faible différence pour les écarts entre le type Sandfelchen et le type Balchen. Cependant, là encore, l'anomalie du Renke du Chiemsee peut s'interpréter comme un mélange ou une hybridation d'autant plus que la variance est telle que cette population pourrait tout aussi bien figurer au tableau no 7 .

Dans le tableau no 8 , populations altérées, il ne subsiste plus que deux groupes. Les populations remaniées par l'homme ou celles qui ont éventuellement subi les vicissitudes de mélanges naturels (auxquels je ne crois guère, en tout cas pour les Corégones alpins) se groupent autour des moyennes du type Balchen et du type Gangfisch. J'ai longtemps admis que les moyennes voisines de 29 branchiospines étaient celles de populations mélangées ou hybridées, parce que c'est d'évidence le cas pour les Corégones d'Italie par exemple.

Je dois reconnaître aussi que le Balchen, forme relativement 


\section{Tableau 6}

Populations pures ou relativement pures (variance jusqu'à 4 au maximum)

\begin{tabular}{|c|c|c|c|c|c|c|}
\hline d'ordre & Population & Lac & $\begin{array}{l}\text { Branchio- } \\
\text { spines }\end{array}$ & $\begin{array}{l}\text { Va- } \\
\text { riance }\end{array}$ & $\begin{array}{c}\text { Coeff. } \\
\text { de } \\
\text { variab. }\end{array}$ & "Ecarts" \\
\hline \multicolumn{7}{|c|}{ Type Kilch } \\
\hline 21 & Storsik & Näckten & $20,7 \pm 0,22$ & 2,63 & 7,82 & 3,1 \\
\hline 61 & Kropfer & Thoune & $20,8 \pm 0,46$ & 1,69 & 6,26 & 3,9 \\
\hline 1 & Kilch & Constance & $21,1 \pm 0,17$ & 2,45 & 7,43 & 3,2 \\
\hline 47 & Storsik & Ismundsjön & $21,9 \pm 0,47$ & 2,89 & 7,78 & 3,4 \\
\hline 2 & Storsik & Storsjön & $22 \pm 0,20$ & 2,33 & 6,94 & 3,8 \\
\hline \multicolumn{7}{|c|}{ Type Sandfelchen } \\
\hline 56 & Grosse Maräne & Selentersee & $24,2 \pm 0,16$ & 2,86 & 6,98 & 4,5 \\
\hline - & Féra ancienne & Léman & $24,3 \pm 0,65$ & 2,25 & 6,10 & $(5,1)$ \\
\hline $20 p$ & Sandfelchen (part.) & Constance & $24,7 \pm 0,31$ & 3,31 & 7,38 & 4,5 \\
\hline 48 & Renke & Chiemsee & $25,7 \pm 0,15$ & 4,05 & 7,82 & 5,2 \\
\hline \multicolumn{7}{|c|}{ Type Balchen } \\
\hline 15 & Storsik & Landösjön & $28,5 \pm 0,30$ & 2,17 & 5,17 & 5,1 \\
\hline 9 & Schnäpel & Ringköbbing & $29,1 \pm 0,19$ & 3,07 & 6,02 & 5,8 \\
\hline 31 & Balchen & Thoune & $29,1 \pm 0,18$ & 3,43 & 6,36 & 5,0 \\
\hline \multicolumn{7}{|c|}{ Type Blaufelchen } \\
\hline 3 & Powan & Lomond & $33,9 \pm 0,08$ & 2,74 & 4,90 & 6,7 \\
\hline 12 & Renke & Ammersee & $35,4 \pm 0,28$ & 3,14 & 5,00 & 7,9 \\
\hline 25 & Storsik & Fullsjön & $36,0 \pm 0,37$ & 2,81 & 4,68 & 7,9 \\
\hline 34 & Storsik & Skällbrägden & $36,2 \pm 0,23$ & 3,80 & 5,38 & 6,0 \\
\hline 51 & Blåsik & Bolesjön & $36,3 \pm 0,38$ & 2,89 & 4,69 & 7,5 \\
\hline 33 & Blaufelchen & Constance (Obersee) & $36,3 \pm 0,11$ & 3,47 & 5,14 & 7,6 \\
\hline 36 & Gråsik & Revsundsjön & $36,6 \pm 0,22$ & 3,69 & 5,26 & 7,3 \\
\hline $6 \mathrm{~B}$ & Skelly & Ullswater & $37,1 \pm 0,21$ & 2,95 & 4,31 & 8,2 \\
\hline 7 & Gwyniad & Bala (Tegid) & $37,3 \pm 0,40$ & 3,76 & 5,20 & 8,1 \\
\hline $44 \mathrm{~A}$ & Reinanke (triés) & Hallstättersee & $37,7 \pm 0,34$ & 2,36 & 4,08 & 7,7 \\
\hline $6 \mathrm{~A}$ & Skelly & Haweswater & $37,7 \pm 0,30$ & 3,11 & 4,69 & 9,0 \\
\hline \multicolumn{7}{|c|}{ Type Gangfisch } \\
\hline 5 & Pollan & Lough Neagh & $3,82 \pm 0,14$ & 2,03 & 3,73 & 9,3 \\
\hline 39 & Småsik & Näckten & $39,2 \pm 0,23$ & 2,42 & 3,96 & 9,2 \\
\hline 40 & Gwyniad & Bala (Tegid) & $39,5 \pm 0,23$ & 2,67 & 4,14 & 8,1 \\
\hline 23 & Gangfisch & Constance (Untersee) & $40,3 \pm 0,19$ & 3,96 & 4,87 & 9,5 \\
\hline 21 & Brienzlig & Brienz & $41,1 \pm 0,2$ & 3,84 & 4,77 & 9,7 \\
\hline 57 & Renke & Riegsee & $42,0 \pm 0,28$ & 3,92 & 4,71 & 9,5 \\
\hline \multicolumn{7}{|c|}{ Type albula } \\
\hline 4 & Pollan & Lough Erne & $41,7 \pm 0,20$ & 3,49 & 4,67 & \\
\hline 58 & Kleine Maräne & Dieksee & $42,6 \pm 0,19$ & 3,71 & 4,50 & 13,5 \\
\hline 59 & " & Pönitzersee & $43,0 \pm 0,17$ & 2,75 & 3,90 & 13,8 \\
\hline 53 & " & Schalsee & $43,2 \pm 0,34$ & 2,54 & 3,69 & 13.9 \\
\hline 55 & Corégone importé & Chauvet & $46,2 \pm 0,42$ & 3,97 & 4,31 & 13,0 \\
\hline
\end{tabular}

Rev. Suisse de Zool., T. 66, 1959. 
TABLEAU 7

Populations relativement peu modifiées par mélanges ou introgression (variances entre 4 et 6 )






\section{TABLEAU 8}

Populations altérées par mélanges ou hybridations

(variances supérieures à 6)

Type Kilch. - Aucune

Type Sandfelchen. - Aucune

Formes intermédiaires à moyennes du type Balchen

\begin{tabular}{l|l|l|r|r|r|r}
\hline 27 & Älvsik & Storsjön (Ytterån) & $26,9 \pm 0,31$ & 9,70 & $\mathbf{1 1 , 5 9}$ & 4,8 \\
45 & Coregono & Vivarone & $27,2 \pm 0,22$ & 7,01 & 9,76 & 5,1 \\
30 & Balchen & Zoug & $27,4 \pm 0,35$ & 8,43 & 10,61 & 5,0 \\
29 & Palée & Bienne & $27,5 \pm 0,34$ & 9,74 & 11,35 & 5,8 \\
62 & Renke & Walchensee & $29,8 \pm 0,49$ & 6,52 & 8,57 & - \\
46 & Blåsik & Storsjön (Änge) & $30,4 \pm 0,27$ & 14,34 & 12,47 & 5,9 \\
60 & "Lavaret " & Annecy & $31,6 \pm 0,14$ & 10,17 & 10,11 & 7,2 \\
24 & Coregono bianco & Majeur & $31,7 \pm 0,25$ & 8,05 & 8,97 & 7,0 \\
& & & & &
\end{tabular}

Type Blaufelchen - Aucune

Type Gangfisch

\begin{tabular}{l|l|l|r|r|r|r|}
\hline & & & \\
17 & Lavaret & Bourget & $38,1 \pm 0,31$ & 9,38 & 8,04 & 9,2 \\
42 & Gangfisch & Constance (Obersee) & $38,4 \pm 0,26$ & 8,66 & 7,67 & 8,9 \\
11 & Renke & Würmsee & $38,5 \pm 0,22$ & 6,16 & 6,45 & 8,8 \\
38 & Lavaret & Aiguebelette & $39,2 \pm 0,31$ & 7,24 & 6,87 & 9,7 \\
44 & Reinanke (non triés) & Hallstättersee & $41,7 \pm 0,33$ & 9 & 7,19 & 10,0 \\
43 & Smärling & Locknesjön & & \\
& & & \\
\hline
\end{tabular}

pure du lac de Thoune - qui serait encore plus pure si l'on avait pas introduit de la Palée du lac de Neuchâtel qui fraie dans les mêmes conditions — pourrait logiquement être considéré comme un hybride de Sandfelchen et de Blaufelchen, deux types qui manquent dans ce lac alors que le Balchen y cohabite avec un Gangfisch (l'Albock) et un Kilch (le Kropfer). Mais cette interprétation, la fusion du Sandfelchen et du Blaufelchen, se heurte au 
fait que justement ces deux espèces paraissent le moins aptes à se mélanger, du fait de leurs habitudes reproductrices, l'une frayant au bord sous peu d'eau, l'autre au large ou en profondeur. Le Balchen fraie au bord sur fond caillouteux. Il faudrait alors admettre que cette fusion aurait été antérieure à l'installation des habitudes reproductrices actuelles, c'est-à-dire très ancienne. Sinon, comment expliquer pourquoi dans ce lac de Thoune, Blaufelchen et Sandfelchen n'ont pas conservé leur autonomie, comme ailleurs ? S'ils ont fusionné naturellement, c'est qu'ils n'étaient que deux sousespèces et le système s'effondre qui subdivise le genre Coregonus en 5 ou 6 espèces distinctes. Cette remarque vaut aussi pour le Schnäpel danois, si semblable au Balchen du lac de Thoune: si le long des côtes de la Suède et du Danemark deux formes distinctes ont fusionné par places pour produire un type intermédiaire comme le Schnäpel, c'est que ces deux formes n'avaient pas atteint le statut spécifique.

L'éventualité d'une fusion complète Sandfelchen $\times$ Blaufelchen étant admise, comment expliquer que la variabilité du nombre des branchiospines chez le produit, Balchen ou Schnäpel, soit du même ordre de grandeur que celui des formes souches ? Cette variabilité devrait être nettement supérieure, comme celle des populations du tableau $n^{0} 8$, celle, par exemple du Balchen du lac de Zoug dont on peut ètre certain qu'il est bien, lui, un produit d'hybridation Sandfelchen $\times$ Blaufelchen, compliqué d'ailleurs d'apports extérieurs. Est-ce qu'un produit hybride, à la longue tend à une réduction de cette variabilité par élimination progressive des cas extrêmes ? Le type Balchen serait alors soit une forme originelle comparable aux autres types, soit le produit ancien d'une fusion, dans certaines conditions particulières, de deux espèces déjà constituées, mais non parfaitement isolées génétiquement, et par conséquent la reconstitution, en quelque sorte d'une espèce antérieurement existante. Ce type se présente biométriquement comme une forme distincte, capable de se maintenir sympatriquement avec d'autres formes voisines, et, par conséquent, selon la doctrine à laquelle je me rattache, il constitue, lui aussi, une espèce valable.

Quant aux populations plus ou moins composites qui conservent plus ou moins le type Gangfisch, je pense qu'il peut s'agir surtout d'introgressions plus ou moins prononcées de formes sympatriques 
avec le Gangfisch et qui fraient, comme lui, sur les bords, au moins dans nos lacs alpestres, c'est-à-dire, selon les cas, de Sandfelchen ou de Balchen. Mais ce sont presque toujours des produits artificiels dus aux pratiques piscicoles.

\section{Prise EN CONSIDÉRATION DU RAPPORT BRANCHIOSPINE SUR ARC}

Le classement en fonction du nombre des branchiospines et de la longueur relative des branchiospines exprimée par les écarts, définis p. 13, aboutit à distinguer les formes à faible dispersion du tableau no 6 et à la possibilité de sélectionner, en se basant sur les variances, celles de ces formes qui paraissent le plus typiques. On peut pourtant objecter que cette sélection et la classification qui en résulte reposent essentiellement sur un seul caractère, celui du nombre des branchiospines. Il y a en effet corrélation entre le nombre des branchiospines et celui des écarts, puisque celui-ci est d'autant plus grand que les branchiospines sont plus serrées. Cette corrélation est pourtant en partie masquée par la tendance, observée subjectivement, qu'ont les branchiospines, au sein d'une population, à être d'autant plus longues qu'elles sont moins nombreuses.

J'ai vérifié cette impression pour la Grande Marène du Selentersee, par exemple. Le coefficient de corrélation entre le nombre de branchiospines et les écarts s'est trouvé égal à $+0,38$ s e u le $\mathrm{m}$ e $\mathrm{n} \mathrm{t}$ (pour 40 individus). J'ai alors exprimé la longueur relative des branchiospines en calculant le rapport de la longueur de la plus longue d'entre elles (normaiement, celle qui occupe l'angle du premier arc) à la longueur de la partie inférieure de cet arc. En abrégé, rapport branchiospine sur arc. Le coefficient de corrélation entre le nombre de branchiospines et les valeurs de ce rapport est: $r=-0,28$. Il me semble indiquer une nette corrélation inverse. Järvi, qui avec Thienemann tient aussi compte de la longueur relative des branchiospines, mais la calcule différemment, signale qu'elle est indépendante de l'âge. J'ai en effet vérifié l'absence totale de corrélation avec la taille des individus dans une population pure. Ces constatations me paraissent donner au caractère de la longueur des branchiospines une valeur systématique intéressante. Il est seulement fâcheux que lesdites branchiospines soient parfois, 
surtout dans certains contingents, déformées, tordues, réduites ou rabougries, de sorte que la plus grande, celle de l'angle, ne peut souvent pas être utilisée pour le calcul du rapport. Je soupçonne ces lésions d'avoir une origine parasitaire, mais l'hôte probable m'est inconnu.

Lorsque ces lésions abondent dans une population comme chez le Gwyniad du lac Bala, la moyenne du rapport en est abaissée d'une quantité difficile à apprécier. Pour le Gwyniad, il semble qu'elle est égale, à peu près, à la différence normale entre le type Blaufelchen et le type Gangfisch.

Si, à l'intérieur d'une population, on peut constater une corrélation inverse entre le nombre des branchiospines et leur longueur, il en va tout autrement quand on compare les diverses populations où la corrélation directe est évidente. Pour le prouver, j’ai calculé la corrélation de rang pour ces deux valeurs sur les populations relativement pures du tableau $n^{0} 6$. Le coefficient obtenu est $r=+0,945$.

Faute de mieux, le rapport branchiospine sur arc m'a donc paru constituer un caractère supplémentaire utile pour distinguer statistiquement les diverses formes. Il présente un inconvénient: la dispersion des valeurs est si élevée, dans chaque population, qu'il en résulte des chevauchements considérables des courbes de variations. Ce caractère à lui seul permet bien de sérier les populations, mais ne suffit pas à les distinguer.

Etant obligé d'établir les valeurs du rapport branchiospine sur arc dans une soixantaine de populations différentes, j'ai limité à 50 (parfois 51) a u $\mathrm{max} \mathrm{i} \mathrm{m} \mathrm{u} \mathrm{m}$ le nombre des individus entrant en ligne de compte pour chaque population. J'ai naturellement choisi alors autant que possible, lorsque je disposais de plusieurs pêches et de contingents importants, les séries obtenues sur les frayères. Les contingents capturés en périodes de fraie offrent certainement moins de risque de mélanges fortuits d'espèces différentes. Dans les tableaux qui suivent, j'ai repris la plupart des populations des tableaux $\mathrm{n}^{\mathrm{os}} 6$ à 8 . J'ai pourtant éliminé le Gangfisch de l'Obersee, manifestement moins pur que celui de l'Untersee (les contingents n'ont pas été pris sur les frayères et comprennent sûrement de jeunes Blaufelchen). J'ai de même laissé de côté quelques populations dont la nature composite et artificielle ne faisait aucun doute. 


\section{Tableau 9}

Branchiospines et rapports br. sur arc

$1^{\text {er }}$ groupe: Populations pures ou relativement pures

(Variance du nombre de Branchiospines jusqu'à 4.)

\begin{tabular}{|c|c|c|c|c|c|c|}
\hline d'ordre & Population & Lac & $\begin{array}{l}\text { Branchio- } \\
\text { spines }\end{array}$ & $\begin{array}{l}\text { Va- } \\
\text { riance }\end{array}$ & $\begin{array}{l}\text { Rapport } \\
\text { br./arc }\end{array}$ & $\begin{array}{l}\text { Va- } \\
\text { riance }\end{array}$ \\
\hline \multicolumn{7}{|c|}{ Type Kilch } \\
\hline 26 & Storsik & Näckten & $20,94 \pm 0,21$ & 2,22 & $23,92 \pm 0,46$ & 10,83 \\
\hline 61 & Kropfer & Thoune & $21,00 \pm 0,50$ & 2,22 & $28,78 \pm 0,94$ & 7,95 \\
\hline 1 & Kilch & Constance & $21,04 \pm 0,20$ & 1,95 & $24,70 \pm 0,44$ & 9,85 \\
\hline 2 & Storsik & Storsjön & $22,20 \pm 0,22$ & 2,40 & $26,24 \pm 0,40$ & 8,06 \\
\hline 47 & Storsik & Ismundsjön & $22,20 \pm 0,50$ & 3,71 & $24,15 \pm 0,62 \mid$ & 5,05 \\
\hline \multicolumn{7}{|c|}{ Type Sandfelchen } \\
\hline $20 p$ & $\begin{array}{l}\text { Sandfelchen } \\
\text { (part.) }\end{array}$ & Constance & $24,29 \pm 0,31$ & 3,05 & 一 & - \\
\hline 20 & & & $24,33 \pm 0,28$ & 3,51 & $28,71 \pm 0,51$ & 8,01 \\
\hline 56 & Grosse Maräne & Selentersee & $24,46 \pm 0,24$ & 2,89 & $28,00 \pm 0,39$ & 7,48 \\
\hline 48 & Renke & Chiemsee & $25,60 \pm 0,25$ & 3,16 & $31,20 \pm 0,49 \mid$ & 11,9 \\
\hline \multicolumn{7}{|c|}{ Type Balchen } \\
\hline 18 & Palée & Léman & $26,82 \pm 0,28$ & 3,79 & $32,60 \pm 0,46$ & 10,64 \\
\hline 49 & & Neuchâtel & $26,94 \pm 0,27$ & 3,54 & $32,00 \pm 0,45$ & 9,92 \\
\hline 15 & Storsik & Landösjön & $28,80 \pm 0,31$ & 2,40 & $29,52 \pm 0,76$ & 14,40 \\
\hline 9 & Schnaepel & Ringköbbing & $28,88 \pm 0,23$ & 2,71 & $31,28 \pm 0,43$ & 9,20 \\
\hline 31 & Balchen & Thoune & $29,54 \pm 0,27$ & 3,69 & $|30,76 \pm 0,47|$ & 10,82 \\
\hline \multicolumn{7}{|c|}{ Type Blaufelchen } \\
\hline 50 & Bondelle & Neuchâtel & $34,2 \pm 0,26$ & 3,48 & $|36,60 \pm 0,38|$ & 7,20 \\
\hline 3 & Powan & Lomond & $34,22 \pm 0,21$ & 2,29 & $31,66 \pm 0,41$ & 8,38 \\
\hline 25 & Storsik & Fullsjön & $35,95 \pm 0,37$ & 2,81 & $34,62 \pm 0,56$ & 6,52 \\
\hline 33 & Blaufelchen & $\begin{array}{l}\text { Constance } \\
\text { (Obersee) }\end{array}$ & $36,58 \pm 0,25$ & 3,05 & $34,96 \pm 0,44$ & 9,43 \\
\hline 36 & Gråsik & Revsundsjön & $36,80 \pm 0,29$ & 3,75 & $36,24 \pm 0,38$ & 10,89 \\
\hline 14 & Småsik & Sundsjön & $37,30 \pm 0,28$ & 3,81 & $33,60 \pm 0,37$ & 6,92 \\
\hline B B & Skelly & Ullswater & $37,41 \pm 0,24$ & 2,95 & $33,76 \pm 0,45$ & 10,06 \\
\hline $6 \mathrm{~A}$ & $"$ & Haweswater & $37,97 \pm 0,33$ & 3,59 & $38,37 \pm 0,57 \mid$ & 10,46 \\
\hline \multicolumn{7}{|c|}{ Type Gangfisch } \\
\hline 5 & Pollan & Lough Neagh & $38,24 \pm 0,18$ & 1,66 & $|37,12 \pm 0,40|$ & 7,87 \\
\hline 39 & Småsik & Näcken & $39,51 \pm 0,28$ & 3,32 & $40,23 \pm 0,51$ & 11,2 \\
\hline 40 & Gwyniad & Bala & $39,71 \pm 0,23$ & 2,54 & $34,69 \pm 0,53$ & 13,30 \\
\hline 23 & Gangfisch & Untersee & $41,02 \pm 0,27$ & 3,78 & $39,22 \pm 0,37$ & 6,81 \\
\hline 21 & Brienzlig & Brienz & $41,78 \pm 0,24$ & 2,85 & $|41,88 \pm 0,51|$ & 13,05 \\
\hline \multicolumn{7}{|c|}{ Type Albula } \\
\hline $\begin{array}{r}4 \\
-8\end{array}$ & Pollan & Lough Erne & $42,56 \pm 0,24$ & 2,85 & $46,06 \pm 0,4$ & 8,06 \\
\hline 58 & Kleme Maräne & Dieksee & $43,02 \pm 0,25$ & 3,14 & $48,26 \pm 0,42$ & 8,99 \\
\hline 83 & $"$ & Schalsee & $43,48 \pm 0,38$ & 3,01 & $49,57 \pm 0,92$ & 17,61 \\
\hline 59 & ". & Pönitzersee & $43,48 \pm 0,26$ & 3,45 & $48,92 \pm 0,51$ & 12,87 \\
\hline 55 & Corég. importé & Lac Chauvet & $46,18 \pm 0,41$ & 3,67 & $|47,45 \pm 0,72|$ & 11,43 \\
\hline
\end{tabular}


TABleau 10

Branchiospines et rapports br. sur arc

$2^{\text {e }}$ groupe: Populations relativement peu modifiées

(Variance du nombre de Branchiospines entre 4 et 6. )

\begin{tabular}{|c|c|c|c|c|c|c|}
\hline $\begin{array}{l}\text { No } \\
\text { d'ordre }\end{array}$ & Population & Lac & $\begin{array}{l}\text { Branchio- } \\
\text { spines }\end{array}$ & $\begin{array}{l}\text { Va- } \\
\text { riance }\end{array}$ & $\begin{array}{l}\text { Rapport } \\
\text { br./arc }\end{array}$ & $\begin{array}{l}\text { Va- } \\
\text { riance }\end{array}$ \\
\hline \multicolumn{7}{|c|}{ Type Kilch. - Aucune } \\
\hline \multicolumn{7}{|c|}{ Type Sandfelchen } \\
\hline 19 & Weissfelchen & $\begin{array}{l}\text { Constance } \\
\text { (Untersee) }\end{array}$ & $25,18 \pm 0,32$ & 5,27 & $29,58 \pm 0,53$ & 13,37 \\
\hline \multicolumn{7}{|c|}{ Type Balchen } \\
\hline $\begin{array}{l}29 \\
10\end{array}$ & $\begin{array}{l}\text { Palée } \\
\text { Reinanke }\end{array}$ & $\begin{array}{l}\text { Bienne } \\
\text { Attersee }\end{array}$ & $\begin{array}{l}26,75 \pm 0,32 \\
29,22 \pm 0,34\end{array}$ & $\begin{array}{l}4,08 \\
5,77\end{array}$ & $\begin{array}{l}32,55 \pm 0,45 \\
35,34 \pm 0,45\end{array}$ & $\begin{array}{r}8,20 \\
10,20\end{array}$ \\
\hline \multicolumn{7}{|c|}{ Type Blaufelchen } \\
\hline $\begin{array}{r}32 \\
12 \\
8 \\
34 \\
51 \\
16\end{array}$ & $\begin{array}{l}\text { Bondelle } \\
\text { Renke } \\
\text { Kröpfling } \\
\text { Storsik } \\
\text { Blåsik } \\
\text { Blaufelchen }\end{array}$ & $\begin{array}{l}\text { Bienne } \\
\text { Ammersee } \\
\text { Attersee } \\
\text { Skällbrägden } \\
\text { Bolesjön } \\
\text { Constance }\end{array}$ & $\begin{array}{l}33,80 \pm 0,32 \\
35,65 \pm 0,33 \\
35,88 \pm 0,30 \\
36,14 \pm 0,31 \\
36,50 \pm 0,46 \\
36,71 \pm 0,31\end{array}$ & $\begin{array}{l}5,20 \\
4,38 \\
4,55 \\
4,96 \\
4,15 \\
4,94\end{array}$ & $\begin{array}{l}33,16 \pm 0,52 \\
37,08 \pm 0,41 \\
37,47 \pm 0,40 \\
28,50 \pm 0,62 \\
36,60 \pm 0,72 \\
36,74 \pm 0,57\end{array}$ & $\begin{array}{r}13,33 \\
6,82 \\
7,98 \\
19,17 \\
10,39 \\
12,46\end{array}$ \\
\hline $\begin{array}{l}52 \\
35 \\
37\end{array}$ & $\begin{array}{l}\text { Älvsik } \\
\text { " } \\
\text { Renke }\end{array}$ & $\begin{array}{l}\text { (Untersee) } \\
\text { Hållstagruppen } \\
\text { Grötingen } \\
\text { Tegernsee } \\
\quad \text { (Grund.) }\end{array}$ & $\begin{array}{l}36,78 \pm 0,39 \\
36,89 \pm 0,30 \\
37,67 \pm 0,35\end{array}$ & $\begin{array}{l}5,95 \\
4,72 \\
5,34\end{array}$ & $\begin{array}{l}34,48 \pm 0,42 \\
38,63 \pm 0,46 \\
36,42 \pm 0,46\end{array}$ & $\begin{array}{r}6,95 \\
10,20 \\
9,22\end{array}$ \\
\hline \multicolumn{7}{|c|}{ Type Gangfisch } \\
\hline $\begin{array}{l}22 \\
38 \\
41 \\
57 \\
43\end{array}$ & $\begin{array}{l}\text { Albock } \\
\text { Lavaret } \\
\text { Älvsik } \\
\text { Renke } \\
\text { Smärling }\end{array}$ & $\begin{array}{l}\text { Thoune } \\
\text { Aiguebelette } \\
\text { Bodsjön } \\
\text { Riegsee } \\
\text { Locknesjön }\end{array}$ & $\begin{array}{l}38,73 \pm 0,37 \\
39,62 \pm 0,30 \\
40,13 \pm 0,44 \\
42,00 \pm 0,29 \\
42,10 \pm 0,30\end{array}$ & $\begin{array}{l}4,20 \\
4,48 \\
5,92 \\
4,39 \\
4,30\end{array}$ & $\begin{array}{l}41,00 \pm 0,39 \\
39,64 \pm 0,45 \\
35,97 \pm 0,54 \\
37,82 \pm 0,50 \\
39,84 \pm 0,42\end{array}$ & $\begin{array}{r}7,44 \\
10,08 \\
8,90 \\
12,69 \\
9,01\end{array}$ \\
\hline \multicolumn{7}{|c|}{ Type Albula } \\
\hline 53 & Kleine Maräne & Plönersee & $43,55 \pm 0,30$ & 4,72 & $48,41 \pm 0,49$ & 12,01 \\
\hline
\end{tabular}




\section{TABLEAU 11}

Branchiospines et rapports br. sur arc

$3^{\mathrm{e}}$ groupe: Populations altérées.

(Variance du nombre de Branchiospines supérieure à 6.)

\begin{tabular}{|c|c|c|c|c|c|c|}
\hline $\begin{array}{l}\text { No } \\
\text { d'ordre }\end{array}$ & Population & Lac & $\begin{array}{l}\text { Branchio- } \\
\text { spines }\end{array}$ & $\begin{array}{l}\mathrm{Va}- \\
\text { riance }\end{array}$ & $\begin{array}{l}\text { Rapport } \\
\text { br./arc }\end{array}$ & $\begin{array}{l}\mathrm{Va}- \\
\text { riance }\end{array}$ \\
\hline
\end{tabular}

Type Kilch. - Aucune

Tupe Sandfelchen. - Aucune

Formes intermédiaires à moyennes voisines du type Balchen

\begin{tabular}{l|l|l|l|l|l|l}
\hline 27 & Älvsik & Storsjön (Ytterån) & $26,4 \mathbf{4} \pm 0,36$ & 6,45 & $28,14 \pm 0,49$ & 11,81 \\
45 & Coregono & Vivarone & $27,01 \pm 0,40$ & 8,04 & $30,66 \pm 0,45$ & 10,00 \\
30 & Balchen & Zoug & $27,40 \pm 0,40$ & 7,80 & $28,76 \pm 0,46$ & 10,70 \\
13 & Älvsik & Alsensjön & $29,20 \pm 0,38$ & 7,20 & $29,02 \pm 0,48$ & 11,54 \\
46 & Blåsik & Storsjön (Änge) & $29,62 \pm 0,60$ & 17,68 & $30,14 \pm 0,71$ & 25,36 \\
& & & & \\
\hline
\end{tabular}

Type Blaufelchen. - Aucune

Type Gangfisch

\begin{tabular}{|c|c|c|c|c|c|c|}
\hline 17 & Lavaret & Bourget & $38,29 \pm 0,44$ & 10,17 & $38,19 \pm 0,42$ & 9,4 \\
\hline 11 & Renke & Würmsee & $38,44 \pm 0,40$ & 7,54 & $37,30 \pm 0,41$ & 8,1 \\
\hline
\end{tabular}

Type albula. - Aucune

On constatera que les données numériques pour le nombre des branchiospines ne sont pas identiques à celles des tableaux précédents. Les différences s'expliquent surtout par le fait que le nombre des individus est parfois fort différent dans ces nouveaux tableaux, dans quelques cas aussi, de petites erreurs de dénombrements ont été soigneusement corrigées.

On notera, en comparant deux à deux les tableaux nos 6 à 8 et $n^{\text {os }} 9$ à 11, le déclassement de quelques populations. Par exemple, le Sandfelchen du lac de Constance, $n^{\circ}$ 20, les Palées du lac de 
Neuchâtel et du Léman, la Bondelle de Neuchâtel passent du deuxième groupe, populations modifiées, au premier groupe, populations relativement pures. La Palée du lac de Bienne passe du troisième au deuxième groupe, comme aussi le Lavaret du Bourget. Dans la plupart des cas, cela signifie simplement, du fait que pour les calculs de rapports j'ai retenu de préférence les individus capturés sur leurs frayères, que les contingents sont plus homogènes en périodes de reproduction, tandis qu'ils comprennent, en périodes d'alimentation, des «intrus», "captés» par les bancs en déplacement.

En revanche, quelques populations passent du premier au second groupe, elles ne sont évidemment pas vraiment pures et leur classement dans l'un ou l'autre groupe peut dépendre du hasard, surtout dans les séries restreintes.

On remarquera également que la distinction des différents types n'est pas tout à fait aussi satisfaisante, quand on considère les rapports, que lorsqu'on prend en considération les écarts.

Le rapport branchiospines sur arc, s'il a l'avantage d'être indépendant du nombre des branchiospines, a le grave inconvénient d'être fluctuant à l'excès. J'ai donc tenté encore un autre moyen de sélection, la combinaison de caractères par l'emploi d'une fonction discriminante.

\section{Recherche D'une Fonction Discriminante}

La recherche d'une fonction discriminante (FIsHer 1946), qui fasse intervenir deux ou plusieurs caractères, a été faite avec l'indispensable collaboration du laboratoire de statistique mathématique de l'Université de Genève. Je tiens à remercier bien vivement son directeur, le professeur Linder, de sa précieuse assistance. Les données fournies aux statisticiens étaient les suivantes: quand on considère les populations chez lesquelles la répartition du nombre des branchiospines est normale, c'est-à-dire gaussienne, on constate que chez certaines, la dispersion des fréquences est nettement plus faible que chez d'autres. J'ai choisi, parmi ces populations que je considère comme plus pures, les deux plus caractéristiques de chaque type, ou, plus exactement, celles qui subjectivement me paraissaient les plus sûres. Pour ces calculs, 
j'ai préféré retenir les formes ou espèces classiques de l'Europe centrale, issues de lacs où elles cohabitent avec d'autres formes plutôt que choisir systématiquement les populations à plus faibles variances, sans doute plus pures, mais dont l'autonomie pourrait ètre mise en doute. J'ai fait une exception pour le Gangfisch dont on obtient, vu le mode de pêche, difficilement des contingents purs, mème sur ses frayères.

Une première tentative de calculer une fonction discriminante a été faite en 1955, en se basant sur l'hypothèse que les populations de Corégones, non compris Coregonus albula, appartenaient aux quatre espèces reconnues par WAGLER. Elle était destinée à éprouver, justement, le système de cet auteur. Le Dr GEIER, qui entreprit pour moi cet essai, et que je remercie bien cordialement, prit en considération quatre caractères: nombre de branchiospines, écarts, nombre d'écailles de la ligne latérale et longueur du corps. Il est inutile d'entrer dans les détails des résultats obtenus, l'analyse prouva qu'une telle constellation de caractères était inutilisable. Le $\mathrm{D}^{\mathrm{r}}$ GeIER refit les calculs en ne prenant plus en considération que le nombre de branchiospines et les écarts. Ces calculs aboutirent à la conclusion que l'analyse basée sur les huit populations retenues ne fournissait pas une définition suffisante des quatre types hypothétiques. Le premier groupe, formé du Kilch et du Storsik du Storsjön était seul bien défini; le deuxième groupe, Sandfelchen de Constance et Reinanke de l'Attersee était particulièrement mauvais, inconsistant, ce qui s'est expliqué ultérieurement puisque ce groupe était composite; le troisième groupe, formé des. Skellies et du Kröpfling de l'Attersee, ni le quatrième, Pollan Erne et Brienzlig, n'étaient satisfaisants; le quatrième groupe en tout cas s'est aussi révélé arbitraire par la suite.

Ces échecs constatés, étant obligé de reprendre le problème à la base, j’ai renoncé aux écarts pour la raison donnée plus haut et calculé les rapports branchiospines sur arc. J'ai déjà dit que, pour économiser du temps et de la peine, les calculs pour soixante populations n'étant pas une petite affaire, j'ai limité à 50 le nombre des individus entrant en ligne de compte pour chaque population.

Pour sélectionner les populations de base, je me suis servi des données des tableaux précédents et des graphiques correspondants. Les populations retenues pour le calcul de la fonction discriminante sont: 


\section{Tableau 13 \\ Fonction discriminante $\mathrm{X} p$}

Populations pures ou relativement pures (variance jusqu'à 50).

\begin{tabular}{|c|c|c|c|c|c|}
\hline d'ordre & Population & Lac & $\mathrm{X} p$ & $\begin{array}{c}\text { Va- } \\
\text { riance }\end{array}$ & $\mathrm{X} p$ du type \\
\hline \multicolumn{6}{|c|}{ Type Kilch } \\
\hline $\begin{array}{r}26 \\
1 \\
47 \\
61 \\
2\end{array}$ & $\begin{array}{l}\text { Storsik } \\
\text { Kilch } \\
\text { Storsik } \\
\text { Kropfer } \\
\text { Storsik }\end{array}$ & $\begin{array}{l}\text { Näckten } \\
\text { Constance } \\
\text { Ismundsjön } \\
\text { Thoune } \\
\text { Storsjön }\end{array}$ & $\begin{aligned} & 97,64 \pm 0,85 \\
& 98,24 \pm 0,76 \\
& 101,08 \pm 1,42 \\
& 102,00 \pm 1,11 \\
& 103,96 \pm 0,79\end{aligned}$ & $\begin{array}{l}35,79 \\
29,06 \\
26,25 \\
11,56 \\
31,28\end{array}$ & $\begin{array}{c}97,86 \\
\text { (Storsik Näckten) } \\
\text { et Kilch. Constance) }\end{array}$ \\
\hline \multicolumn{6}{|c|}{ Type Sandfelchen } \\
\hline $\begin{array}{c}56 \\
20 p \\
48\end{array}$ & $\begin{array}{l}\text { Maräne } \\
\text { Sandfelchen } \\
\text { Renke }\end{array}$ & $\begin{array}{l}\text { Selentersee } \\
\text { Constance } \\
\quad \text { (Obersee) } \\
\text { Chiemsee }\end{array}$ & $\begin{array}{l}113,68 \pm 0,84 \\
113,74 \pm 1,13 \\
120,64 \pm 0,88\end{array}$ & $\begin{array}{l}34,93 \\
39,93 \\
\\
33,80\end{array}$ & $\begin{array}{c}113,80 \\
\text { (Sandfelchen et } \\
\text { Maräne, Selentersee) }\end{array}$ \\
\hline \multicolumn{6}{|c|}{ Type Balchen } \\
\hline $\begin{array}{r}18 \\
15 \\
9\end{array}$ & $\begin{array}{l}\text { Palée } \\
\text { Storsik } \\
\text { Schnäpel }\end{array}$ & $\begin{array}{l}\text { Léman } \\
\text { Landösjön } \\
\text { Ringköbbing }\end{array}$ & $\begin{array}{l}126,32 \pm 0,95 \\
130,32 \pm 1,29 \\
132,96 \pm 0,85\end{array}$ & $\begin{array}{l}45,27 \\
41,50 \\
36,52\end{array}$ & $\begin{array}{c}133,25 \\
\text { (Balchen Thoune } \\
\text { et Schnäpel) }\end{array}$ \\
\hline \multicolumn{6}{|c|}{ Type Blaufelchen } \\
\hline $\begin{array}{c}3 \\
50 \\
25 \\
14 \\
\text { B B } \\
36\end{array}$ & $\begin{array}{l}\text { Powan } \\
\text { Bondelle } \\
\text { Storsik } \\
\text { Småsik } \\
\text { Skelly } \\
\text { Gråsik }\end{array}$ & $\begin{array}{l}\text { Loch Lomond } \\
\text { Neuchâtel } \\
\text { Fullsjön } \\
\text { Sundsjön } \\
\text { Ullswater } \\
\text { Revsundsjön }\end{array}$ & $\begin{array}{l}151,28 \pm 0,80 \\
156,24 \pm 0,92 \\
161,14 \pm 1,38 \\
164,04 \pm 0,93 \\
164,84 \pm 0,85 \\
165,14 \pm 0,97\end{array}$ & $\begin{array}{l}32,12 \\
42,34 \\
39,84 \\
42,96 \\
36,33 \\
41,35\end{array}$ & $\begin{array}{l}164,84 \\
\text { (Blaufelchen Obersee, } \\
\text { Grötingen, Ullswater) }\end{array}$ \\
\hline \multicolumn{6}{|c|}{ Type Gangfisch } \\
\hline $\begin{array}{r}5 \\
40 \\
22 \\
21\end{array}$ & $\begin{array}{l}\text { Pollan } \\
\text { Gwygniad } \\
\text { Albock } \\
\text { Brienzlig }\end{array}$ & $\begin{array}{l}\text { Lough Neagh } \\
\text { Bala } \\
\text { Thoune } \\
\text { Brienz }\end{array}$ & $\begin{array}{l}170,73 \pm 0,71 \\
173,92 \pm 1,02 \\
176,40 \pm 1,25 \\
188,12 \pm 0,91\end{array}$ & $\begin{array}{l}24,93 \\
49,49 \\
46,77 \\
41,38\end{array}$ & $\begin{array}{c}186,45 \\
\text { (Brienzlig et Renke, } \\
\text { Riegsee) }\end{array}$ \\
\hline \multicolumn{6}{|c|}{ Type Albula } \\
\hline $\begin{array}{r}4 \\
58 \\
53\end{array}$ & $\begin{array}{l}\text { Pollan } \\
\text { Kl. Maräne } \\
\text { „ }\end{array}$ & $\begin{array}{l}\text { Lough Erne } \\
\text { Dieksee } \\
\text { Schalsee }\end{array}$ & $\begin{array}{l}194,96 \pm 0,87 \\
198,64 \pm 0,90 \\
201,62 \pm 1,52\end{array}$ & $\begin{array}{l}38,28 \\
40,31 \\
48,34\end{array}$ & $\begin{array}{c}199,97 \\
\text { (Pollan Erne, } \\
\text { Dieksee et Pönitzersee) }\end{array}$ \\
\hline
\end{tabular}




\section{TABLEAU 14 \\ Fonction discriminante $\mathrm{X} p$}

Populations relativement peu modifiées (variances entre 50 et 80 ).

\begin{tabular}{|c|c|c|c|c|}
\hline $\begin{array}{l}\text { No } \\
\text { d'ordre }\end{array}$ & Population & Lac & $\mathrm{X} p$ & Variance \\
\hline \multicolumn{5}{|c|}{ Type Kilch. - Aucune } \\
\hline \multicolumn{5}{|c|}{ Type Sandfelchen } \\
\hline $\begin{array}{l}20 \\
19\end{array}$ & $\begin{array}{l}\text { Sandfelchen } \\
\text { Weissfelchen }\end{array}$ & $\begin{array}{c}\text { Constance Obersee } \\
\| \quad \text { Untersee }\end{array}$ & $\begin{array}{l}114,48 \pm 1,23 \\
120,92 \pm 1,66\end{array}$ & $\begin{array}{l}63,49 \\
71,38\end{array}$ \\
\hline \multicolumn{5}{|c|}{ Type Balchen } \\
\hline $\begin{array}{l}29 \\
49 \\
31\end{array}$ & $\begin{array}{l}\text { Palée } \\
\text { Palée } \\
\text { Balchen }\end{array}$ & $\begin{array}{l}\text { Bienne } \\
\text { Neuchâtel } \\
\text { Thoune }\end{array}$ & $\begin{array}{l}126,10 \pm 1,26 \\
126,28 \pm 1,02 \\
136,16 \pm 1,06\end{array}$ & $\begin{array}{l}63,99 \\
51,68 \\
56,45\end{array}$ \\
\hline \multicolumn{5}{|c|}{ Type Blaufelchen } \\
\hline $\begin{array}{r}32 \\
12 \\
8 \\
51 \\
35 \\
37\end{array}$ & $\begin{array}{l}\text { Bondelle } \\
\text { Renke } \\
\text { Kröpfling } \\
\text { Älvsik } \\
\text { Älvsik } \\
\text { Renke }\end{array}$ & $\begin{array}{l}\text { Bienne } \\
\text { Ammersee } \\
\text { Attersee } \\
\text { Bolesjön } \\
\text { Grötingen } \\
\text { Tegernsee }\end{array}$ & $\begin{array}{l}151,40 \pm 1,24 \\
161,85 \pm 1,21 \\
162,84 \pm 1,04 \\
164,40 \pm 1,75 \\
167,18 \pm 1,18 \\
168,88 \pm 1,29\end{array}$ & $\begin{array}{l}76,68 \\
58,48 \\
53,61 \\
61,04 \\
68,07 \\
71,69\end{array}$ \\
\hline \multicolumn{5}{|c|}{ Type Gangfisch } \\
\hline $\begin{array}{l}6 \mathrm{~A} \\
41 \\
38 \\
39 \\
23 \\
57 \\
43\end{array}$ & $\begin{array}{l}\text { Skelly } \\
\text { Älvsik } \\
\text { Lavaret } \\
\text { Småsik } \\
\text { Gangfisch } \\
\text { Renke } \\
\text { Smärling }\end{array}$ & $\begin{array}{l}\text { Haweswater } \\
\text { Bodsjön } \\
\text { Aiguebelette } \\
\text { Näckten } \\
\text { Untersee } \\
\text { Riegsee } \\
\text { Locknesjön }\end{array}$ & $\begin{array}{l}171,44+1,27 \\
176,47 \pm 1,62 \\
178,04 \pm 1,22 \\
178,51 \pm 1,17 \\
182,49 \pm 1,10 \\
184,64 \pm 1,04 \\
186,12 \pm 1,12\end{array}$ & $\begin{array}{l}51,81 \\
79,12 \\
71,75 \\
58,81 \\
59,35 \\
53,67 \\
62,87\end{array}$ \\
\hline \multicolumn{5}{|c|}{ Type Albula } \\
\hline $\begin{array}{l}54 \\
59 \\
55\end{array}$ & $\begin{array}{l}\text { Kleine Maräne } \\
\text { Kleine Maräne } \\
\text { Corégone importé }\end{array}$ & $\begin{array}{l}\text { Plönersee } \\
\text { Pönitzersee } \\
\text { Chauvet }\end{array}$ & $\begin{array}{l}200,80 \pm 1,05 \\
201,80 \pm 1,02 \\
209,00 \pm 1,66\end{array}$ & $\begin{array}{l}55,20 \\
52,30 \\
61,00\end{array}$ \\
\hline
\end{tabular}


TABleau 15

\section{Fonction discriminante $\mathrm{Xp}$}

Populations altérées par mélanges ou hybridations

(variances supérieures à 80 ).

\begin{tabular}{|c|c|c|c|c|}
\hline d'ordre & Population & Lac & $\mathrm{X} p$ & Variance \\
\hline \multicolumn{5}{|c|}{ Type Kilch. - Aucune } \\
\hline \multicolumn{5}{|c|}{ Type Sandfelchen. - Aucune } \\
\hline \multicolumn{5}{|c|}{ Type Balchen } \\
\hline $\begin{array}{l}27 \\
30 \\
13 \\
10\end{array}$ & $\begin{array}{l}\text { Älvsik } \\
\text { Balchen } \\
\text { Älvisk } \\
\text { Reinanke }\end{array}$ & $\begin{array}{l}\text { Storsjön (Ytterån) } \\
\text { Zoug } \\
\text { Alsensjön } \\
\text { Attersee }\end{array}$ & $\begin{array}{l}121,68 \pm 1,38 \\
124,80 \pm 1,51 \\
131,20 \pm 1,57 \\
137,64 \pm 1,35\end{array}$ & $\begin{array}{r}94,62 \\
114,56 \\
122,72 \\
90,67\end{array}$ \\
\hline \multicolumn{5}{|c|}{ Type Blaufelchen } \\
\hline $\begin{array}{l}34 \\
16\end{array}$ & $\begin{array}{l}\text { Storsik } \\
\text { Blaufelchen }\end{array}$ & $\begin{array}{l}\text { Skällbrägden } \\
\text { Constance(Untersee) }\end{array}$ & $\begin{array}{l}155,56 \pm 1,35 \\
163,16 \pm 1,50\end{array}$ & $\begin{array}{l}89,65 \\
84,98\end{array}$ \\
\hline \multicolumn{5}{|c|}{ Type Gangfisch } \\
\hline $\begin{array}{l}17 \\
11\end{array}$ & $\begin{array}{l}\text { Lavaret } \\
\text { Renke }\end{array}$ & $\begin{array}{l}\text { Bourget } \\
\text { Würmsee }\end{array}$ & $\begin{array}{l}172,19 \pm 1,55 \\
172.98 \pm 1,45\end{array}$ & $\begin{array}{l}128,04 \\
103,53\end{array}$ \\
\hline
\end{tabular}

1er type: no 26, Storsik, Näckten (Suède) et no 1, Kilch (Constance); 2e type: no 20, Sandfelchen, Constance et no 56 , Maräne, Selentersee;

3e type: no 31, Balchen, Thoune et no 9, Schnäpel, Ringköbbing; 4e type: no 33, Blaufelchen, Constance, no 35, Älvsik, Grötingen (S) et no 6 B, Skelly, Ullswater.


vière);

6e type: no 4, Pollan Lough Erne, nos 58 et 59, Kleine Maräne, Dieksee et Pönitzersee (Holstein). 
La fonction obtenue a été: $\mathrm{X} p=3,5 x+y$, dans laquelle $x=$ le nombre des branchiospines et $y$ les valeurs en $\%$ du rapport branchiospines sur arc inférieur. Je tiens à remercier encore M. LANG, assistant, de son aimable collaboration.

Dans les tableaux 13 à 15 , les populations sont classées dans l'ordre des valeurs croissantes de la fonction discriminante. J'ai limité arbitrairement à 50 la variance des populations considérées comme pures ou pratiquement pures (tableau 13). Le tableau 14 comprend les variances de 50 à 80 , j'admets qu'elles correspondent aux populations encore reconnaissables mais nettement modifiées. Au-delà de 80 , on peut considérer que les populations sont nettement altérées (tableau 15).

La combinaison de deux caractères bien différents, quoique se rapportant tous deux aux branchiospines, permet une sélection satisfaisante des populations, séletion qui s'accorde assez bien avec les précédentes, celles des tableaux $n^{\text {os }} 6$ à 8 et $n^{\text {os }} 9$ à 11 .

Je pense qu'on doit donner plus de poids à la fonction discriminante et considérer comme le meilleur le triage auquel on aboutit par ce moyen, malgré que le choix des populations de base n'ait pas été toujours heureux. Un choix plus rigoureux des populations

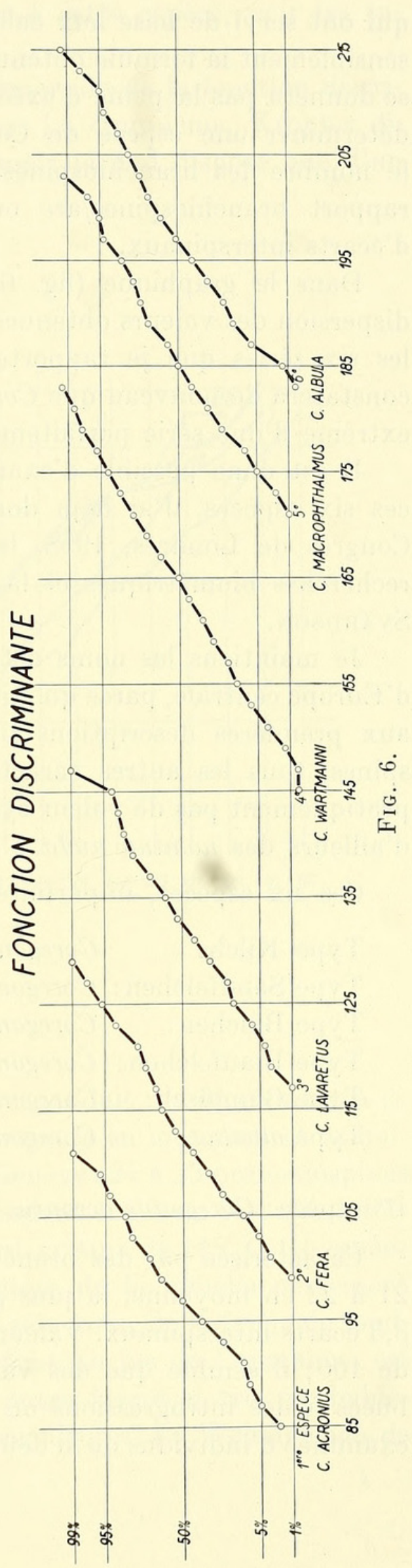


qui ont servi de base aux calculs n'aurait sans doute pas modifié sensiblement la formule obtenue. D'ailleurs, dans la pratique, on ne se donnera pas la peine d'exécuter tous ces calculs, il suffira pour déterminer une espèce de Corégone de prendre en considération le nombre des branchiospines, et leur longueur exprimée par le rapport branchiospines/arc ou plus simplement par le nombre d'écarts interspinaux.

Dans le graphique (fig. 6), on peut apprécier l'allure de la dispersion des valeurs obtenues pour la fonction discriminante dans les six séries que je rapporte à autant d'espèces distinctes. On constatera de nouveau que Coregonus albula se présente comme un extrême d'une série parfaitement cohérente.

Il est donc possible d'examiner et de caractériser chacune de ces six espèces. J'ai déjà donné, lors de ma communication au Congrès de Londres, 1958, le système auquel m'ont amené ces recherches biométriques et la correspondance avec le système de SvärdSON.

Je maintiens les noms actuellement usités pour les Corégones d'Europe centrale, parce qu'à mon sens la priorité doit être accordée aux premières descriptions basées sur le nombre des branchiospines, tous les autres caractères utilisés précédemment n'ayant pratiquement pas de valeur systématique, les formes décrites étant d'ailleurs des nomina nuda.

Ces six espèces, imparfaites, sont donc:

Type Kilch: $\quad$ Coregonus acronius Smitt;

Type Sandfelchen: Coregonus fera Jurine;

Type Balchen: Coregonus lavaretus (L.);

Type Blaufelchen: Coregonus wartmanni (Bloch);

Type Gangfisch: Coregonus macrophthalmus Nüsslin;

Type albula: $\quad$ Coregonus albula (L.).

1re espèce: Coregonus acronius Smitt.

Caractérisée par des branchiospines très courtes et très lâches, 21 à 22 en moyenne, la plus grande correspondant en moyenne à 3,5 écarts interspinaux. Valeur de la fonction discriminante voisine de 100; il semble que des valeurs supérieures doivent être attribuées à des introgressions ou à la présence fortuite dans la série examinée d'individus de la deuxième espèce. Cinq populations pures 
ou relativement pures appartiennent à cette espèce, voir les tableaux 6,9 et 13 .

Le graphique (fig. 7) montre la dispersion de la fonction discriminante pour 4 de ces 5 populations. La cinquième, Kropfer du lac de Thoune en fait aussi partie, mais je n'ai disposé que d'un contingent trop restreint.

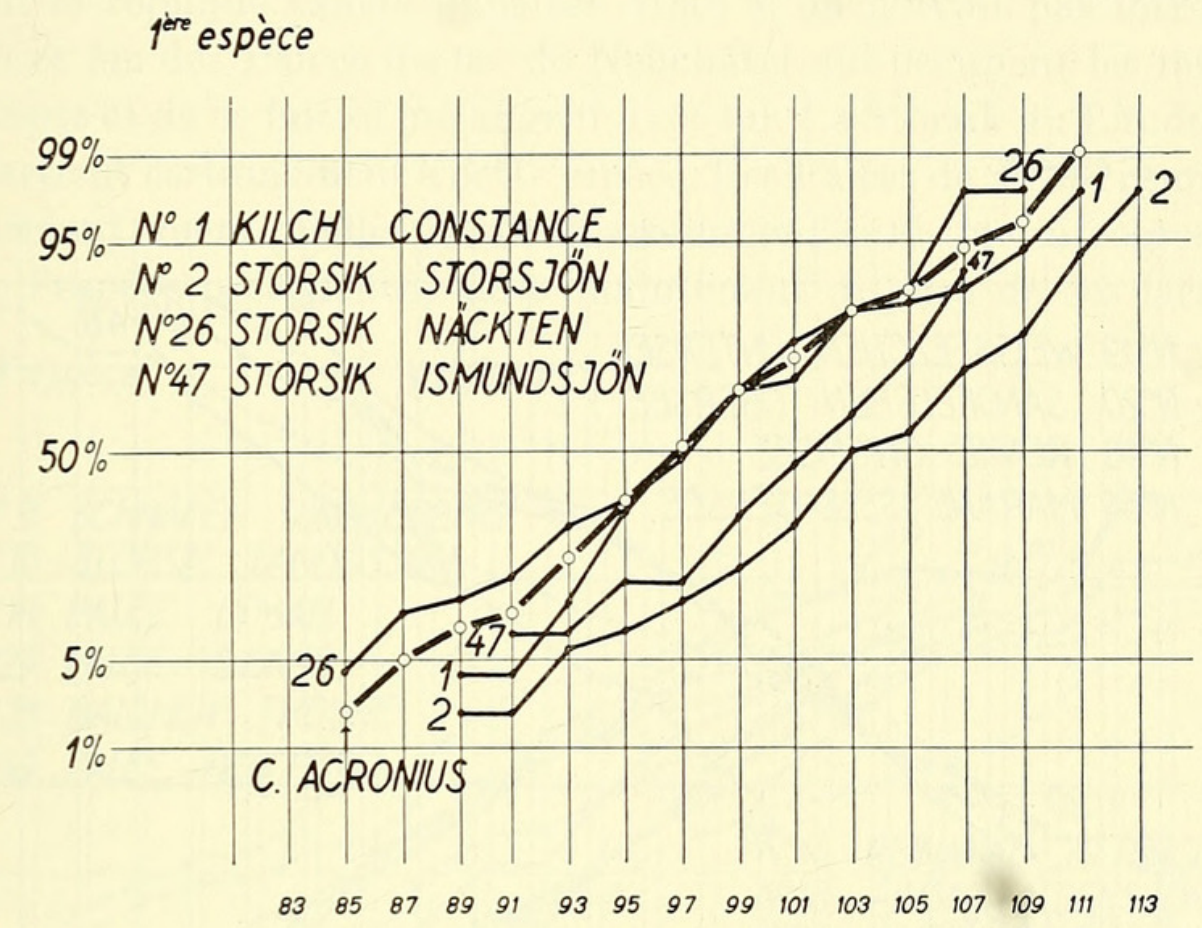

FIG. 7.

$2^{\mathrm{e}}$ espèce: Coregonus fera Jurine.

La véritable Féra du Léman correspondait manifestement à ce type, comme il est facile de s'en convaincre en mesurant et examinant les exemplaires du muséum de Genève: 24 à 25 branchiospines en moyenne, la plus grande correspondant à 4,5 ou 5 écarts interspinaux. La fonction discriminante est voisine de 115. Cette espèce partage avec la précédente le caractère de la bouche nettement inférieure et une tendance évidente à se nourrir de la faune de fond. Le type Sandfelchen, tel qu'il est pêché au lac de Constance, est souvent mélangé d'individus d'une autre forme et très probablement d'hybrides produits par les piscicultures. Le Weissfelchen de 
l'Untersee ne diffère du Sandfelchen que par un mélange constant, surtout avec des individus de la quatrième espèce et la production probable d'une certaine proportion d'hybrides artificiels. Le mode de pêche à la seine et le brassage qui en résulte sont sans aucun doute responsables de ces mélanges.

L'espèce est représentée, apparemment à l'état pur, au Selentersee, c'est la forme décrite par Thienemann sous le nom de C. holsatus.

\section{$2^{e}$ espèce}

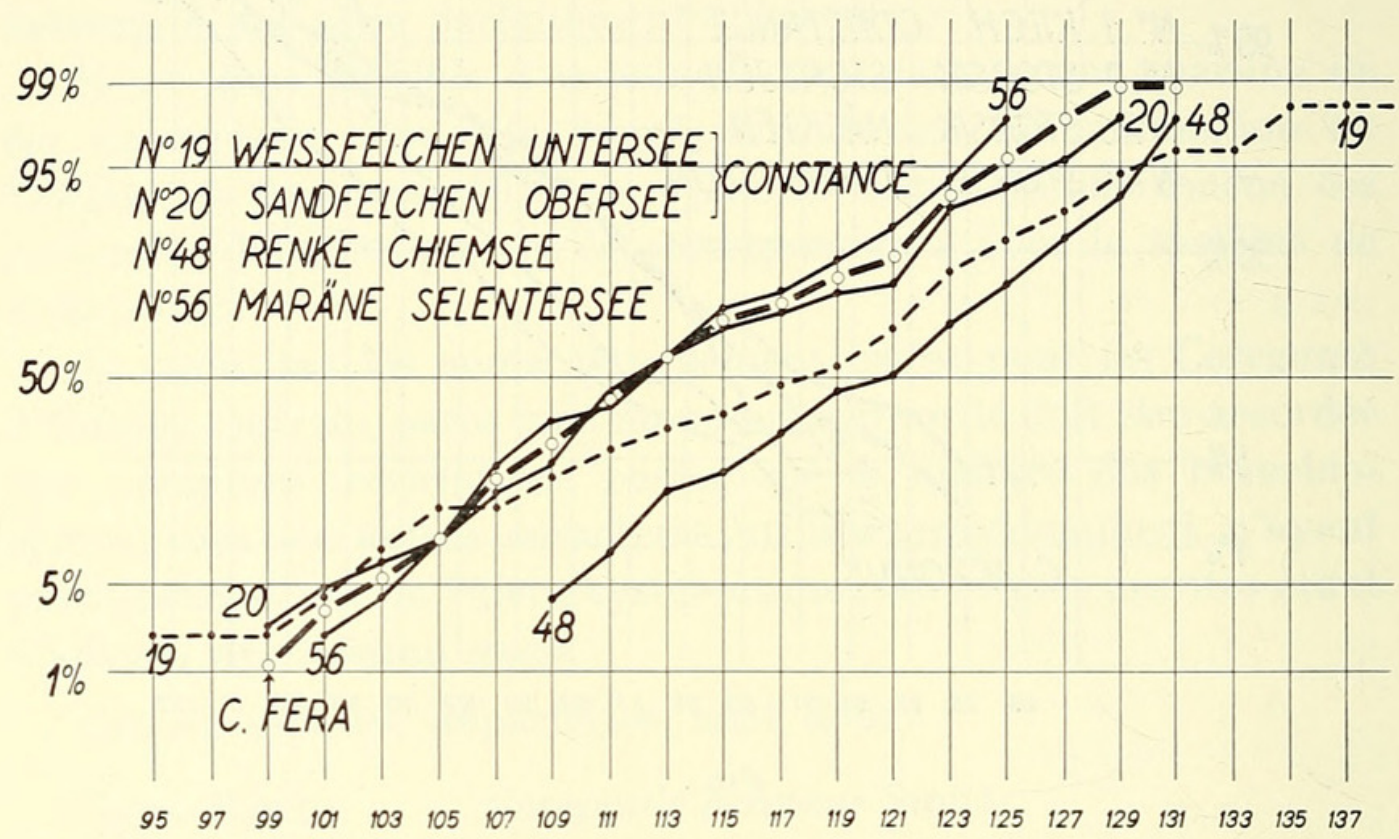

FIG. 8.

Le graphique (fig. 8) représente la dispersion des populations appartenant à cette espèce. En pointillé, le Weissfelchen, manifestement impur. Je n'ai pas assez d'individus de l'authentique Féra du Léman pour que cette forme puisse figurer sur le graphique. La Renke du Chiemsee s'écarte notablement, comme on peut le voir, du type de l'espèce et se rapproche du type suivant.

Ces deux premières espèces, qui sont peut-être en voie de disparition, semblent absentes des eaux intérieures de GrandeBretagne et d'Irlande. 
3e espèce: Coregonus lavaretus (L.).

S'il n'existait pas de populations relativement pures et à faible dispersion pour les caractères considérés, j’aurais considéré le type Balchen comme un produit d'hybridation (voir p. 36). Le Schnäpel du Ringköbbing est en fait la population la plus typique. Nombre de branchiospines 28-29 en moyenne, écarts entre 5 et 6 , fonction discriminante voisine de 130. Le Balchen de Thoune en serait la réplique exacte dans les Alpes si on n'avait pas introduit dans ce lac des Palées du lac de Neuchâtel qui occupent les mêmes biotopes et de ce fait se mélangent avec lui. Le Storsik du Landösjön appartient certainement à cette espèce. Les Palées de Suisse romande également, bien qu'elles aient apparemment subi une introgression avec l'espèce précédente, qui a maintenant disparu de ces lacs.

\section{$3^{e}$ espèce}

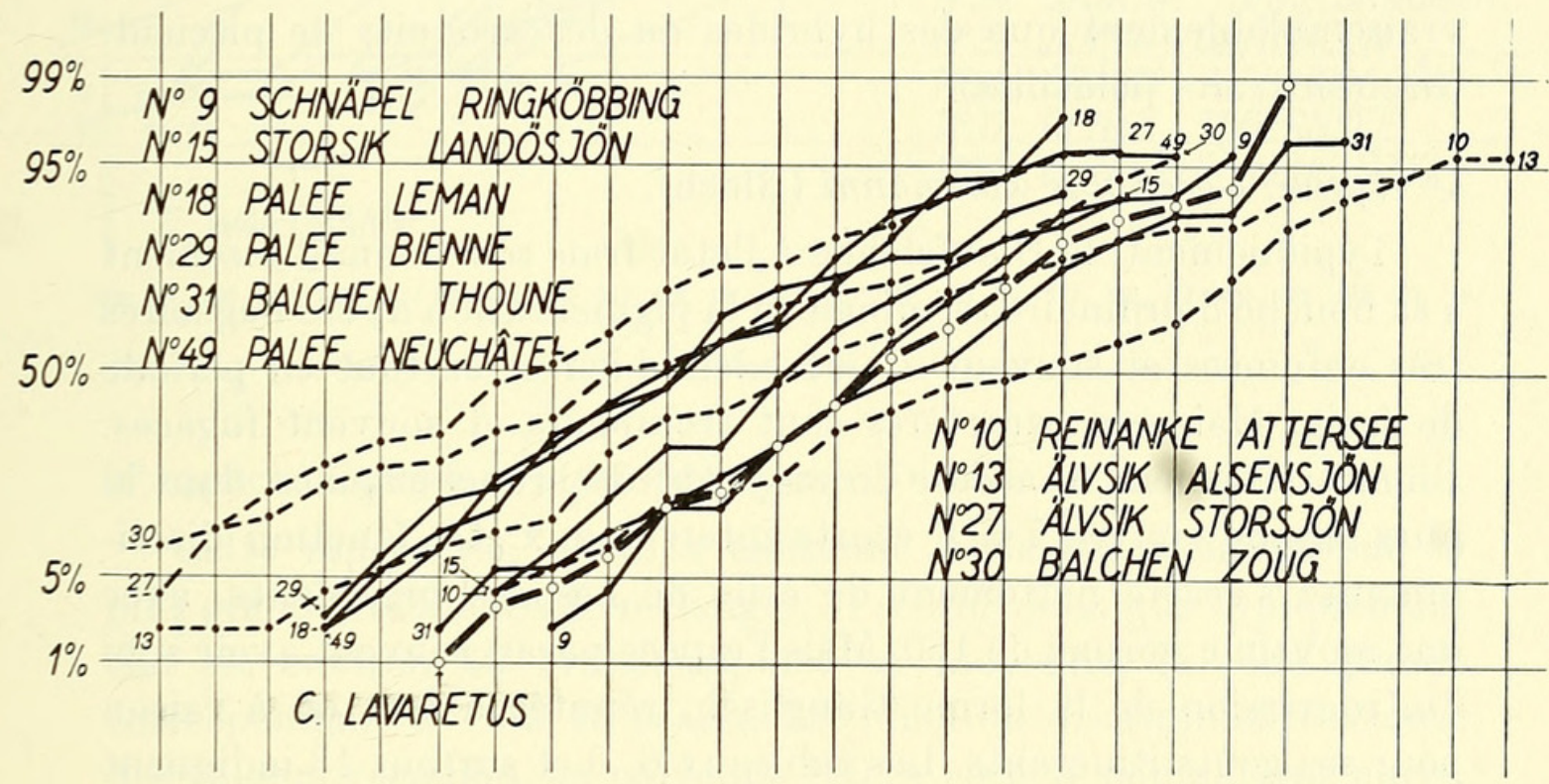

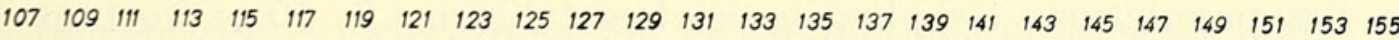

FIG. 9.

Cette espèce présente beaucoup moins que les précédentes le caractère de la bouche inférieure et son alimentation est, en Suisse en tout cas, presque exclusivement planctonique.

A côté des populations appartenant à coup sûr à ce type, on rencontre dans divers lacs des populations accusant le même nombre moyen de branchiospines, voisin de 30, mais qui sont des 
hybrides certains ou des produits de brassages inextricables dues à la pisciculture. Les populations altérées ou composites se reconnaissent à la variance considérable de la fonction discriminante, en particulier. Les Corégones des lacs italiens, le Corégone du lac d'Annecy, le Balchen du lac de Zoug sont des exemples de populations dont la nature complexe ou hybride peut être démontrée. Ce dernier seul, le Balchen de Zoug, est porté sur le graphique (fig. 9). D'autres populations ne sont peut-être qu'en apparence apparentés au type Balchen; ce sont en particulier, l’Älvsik pêché à Ytterån et celui de l'Alsensjön. Ce dernier, avec une variance de la fonction discriminante atteignant la valeur considérable de 122, semble bien une forme artificielle, comme Swärdson me l'avait en effet signalé (voir p. 27).

Le graphique (fig. 9) comprend à la fois des populations appartenant à l'espèce (en traits pleins) et d'autres qui ne sont vraisemblablement que des hybrides ou des produits de pisciculture (en traits pointillés).

4e espèce: Coregonus wartmanni (Bloch).

Typiquement, le Blaufelchen à l'état frais se reconnaît aisément à sa bouche d'ordinaire terminale, à la pigmentation de ses nageoires très enfumées et souvent à sa couleur bleutée surtout en période de fraie. Mais ces caractères sont trompeurs et souvent fugaces. Biométriquement, il accuse en moyenne 36 branchiospines, dont la plus grande couvre 7 à 8 écarts interspinaux. La fonction discriminante s'écarte nettement de celle de l'espèce précédente, avec une moyenne voisine de 160. Mais l'espèce paraît souvent avoir subi l'introgression de la forme Gangfisch, réputée à tort ou à raison pour ses goûts itinérants. Les tableaux 6, 9 et surtout 13 indiquent les populations les plus pures. Les tableaux 7, 10 et surtout 14 comprennent celles qui doivent être regardées comme impures quoique encore reconnaissables.

Le graphique (fig. 10) ne laisse pas d'être troublant par le fait de l'existence, à côté des populations typiques groupées de part et d'autre de la ligne spécifique, d'autres populations dont la valeur pour la fonction discriminante est nettement plus faible. S'agit-il d'une différence entre les $C$. wartmanni dans le nord et dans les Alpes correspondant à l'écart de 2 ou 3 branchiospines entre l'interprétation de Svärdson et la mienne? Ou bien s'agit-il d'intro- 
gression de l'espèce précédente comme cela parait très probable pour la Bondelle? Ou encore, cette dispersion est-elle parfois l'expression d'un phénomène biologique: les malformations fréquentes des branchiospines par lésions parasitaires?

\section{$4^{\circ}$ espèce}

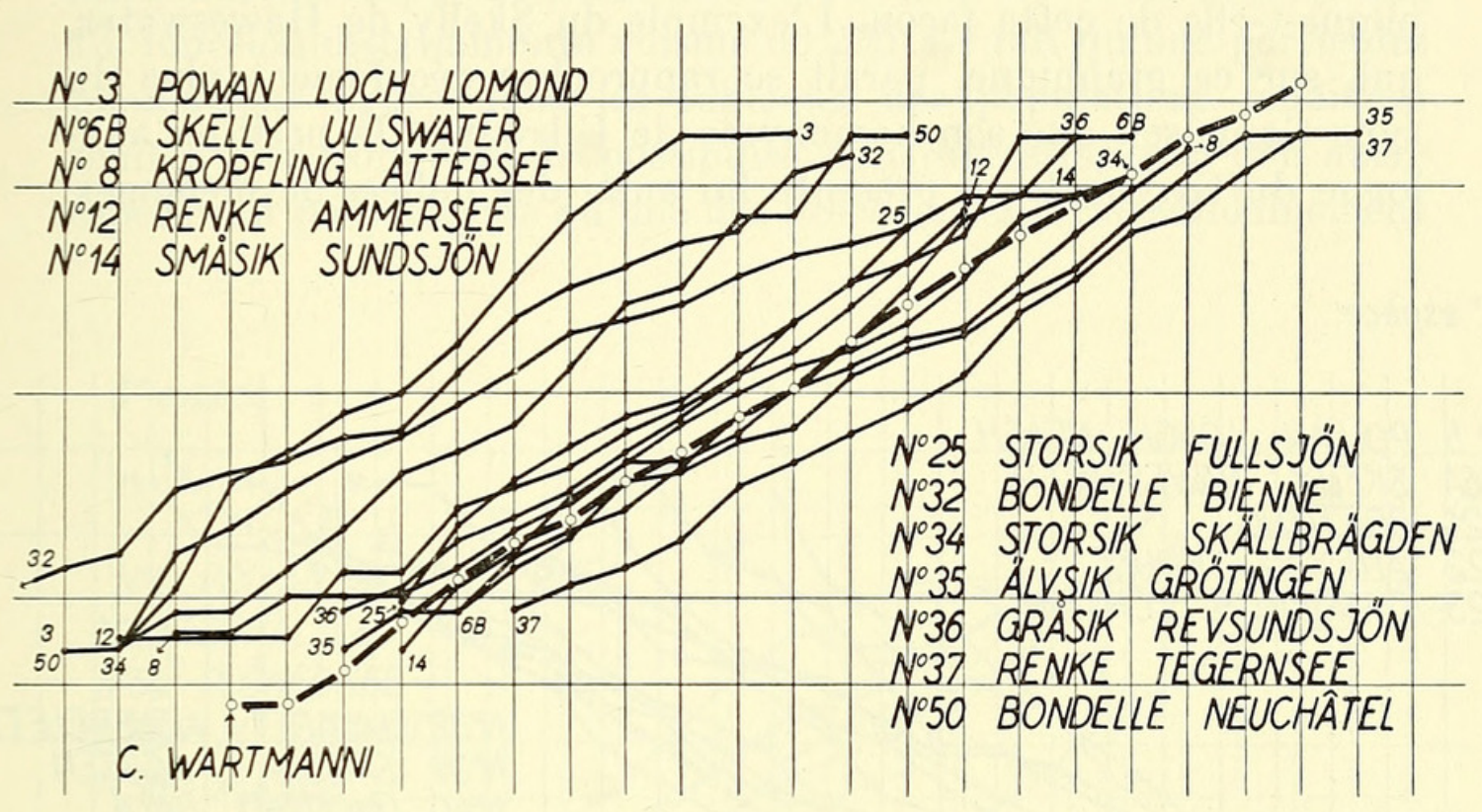

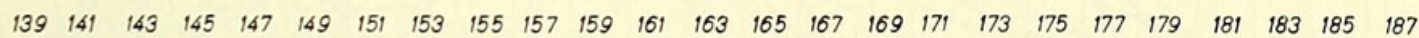

FIG. 10.

5e espèce: Coregonus macrophthalmus Nüsslin.

Cette espèce est, avec la troisième, celle qui témoigne de la plus grande fréquence de mélanges. Il semble bien que typiquement elle ait environ 40 branchiospines en moyenne, la plus grande correspondant à 9 ou 10 écarts. La fonction discriminante accuse des valeurs variables, en moyenne 180. A côté de populations pures, on rencontre une forte proportion de populations nettement modifiées ou profondément altérées. Il me paraît évident qu'on doit à la pisciculture les populations hybrides incontestables comme celle du Würmsee ou le Lavaret du Bourget. On remarquera dans le tableau 14 que le Skelly de Haweswater figure parmi les populations (peu) modifiées de cette espèce tandis qu'il figurait, non sans réticences de ma part, parmi les populations (relativement) pures du tableau 6.

Je suis convaincu qu'une étude plus poussée fixera la nature composite de cette forme. 
Le graphique (fig. 11) semble confirmer que C. macrophthalmus est l'espèce la moins stable; elle me paraît subir, naturellement ou par intervention humaine, de fréquents mélanges, surtout avec les espèces précédentes.

Peut-être la position des Corégones de Grande-Bretagne s'explique-t-elle de cette façon. L'exemple du Skelly de Haweswater, qui, sur ce graphique, parait se rapprocher décidément plus du type Gangfisch que son homonyme de Ullswater, la position analogue du Gwyniad qui présente lui aussi des indices de mélanges,

\section{$5^{e}$ espèce}

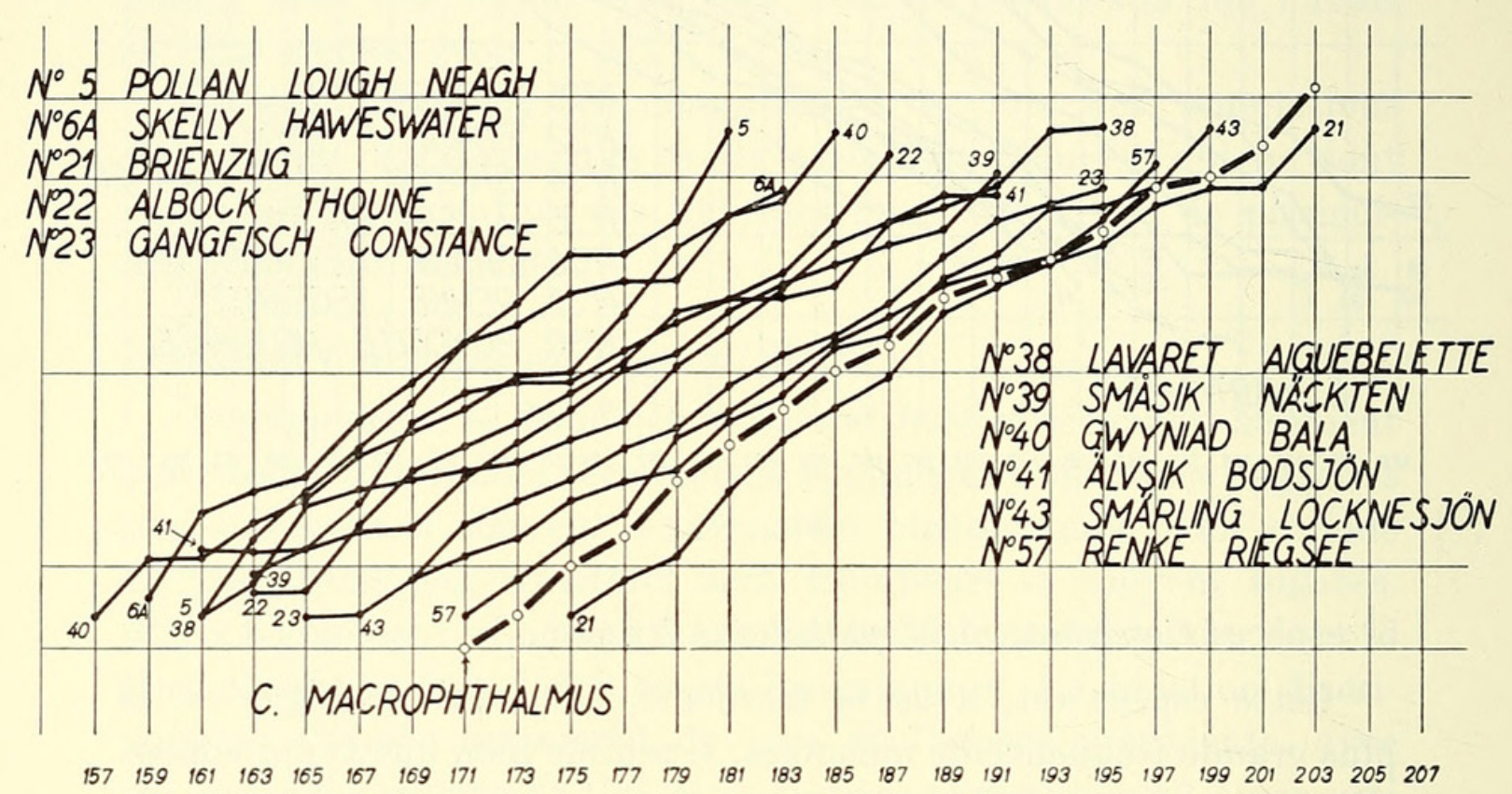

FIG. 11.

parleraient en faveur d'une tendance de cette espèce à absorber plus facilement que les autres des individus étrangers et par conséquent à se modifier plus fréquemment par introgression. Cette espèce est encore celle qui présente le plus souvent des malformations des branchiospines. Mais il se pourrait encore qu'il s'agisse, en Grande-Bretagne, d'un certain endémisme insulaire. Avant d'envisager l'éventualité que ces populations britanniques constituent des sous-espèces particulières, il importe de pousser plus loin et plus à fond l'analyse statistique et biologique de ces formes. 
6e espèce: Coregonus albula (L.).

Reconnue de tout temps, elle ne se distingue vraiment que par sa bouche, d'habitude supère. Branchiospines en moyenne 43, mais la forme importée au lac Chauvet accuse 46, écarts en moyenne 13, fonction discriminante voisine de 200. Le fait qu'une partie des populations figure dans les tableaux des populations modifiées semble bien confirmer la possibilité d'introgression par une autre espèce qui ne peut être qu'une des précédentes, vraisemblablement

$\sigma^{e}$ espèce

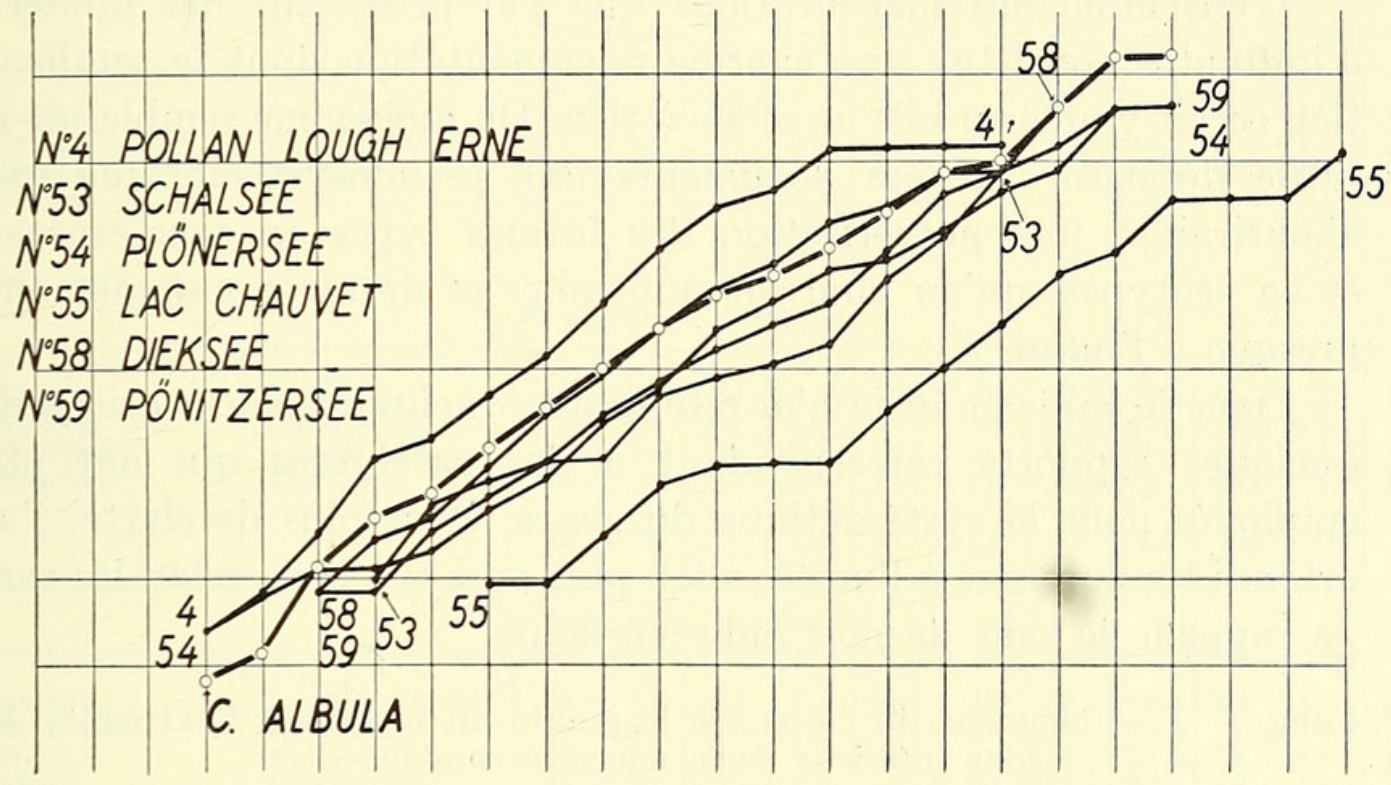

$\begin{array}{lllllllllllllllllllll}181 & 183 & 185 & 187 & 189 & 191 & 193 & 195 & 197 & 199 & 201 & 203 & 205 & 207 & 209 & 211 & 213 & 215 & 217 & 219 & 221\end{array}$

FIG. 12.

la $4^{\mathrm{e}}$ ou la $5^{\mathrm{e}}$, selon les cas. Dans le graphique (fig. 12), la variabilité de cette espèce apparaît analogue à celle de $C$. acronius. Le Pollan du Lough Erne n'est-il qu'un extrême d'une variabilité normale ou une population quelque peu modifiée par mélange avec l'espèce précédente? On remarquera à l'opposé la position insolite du Corégone importé dans le lac Chauvet et dont l'origine est énigmatique (voir à ce sujet l'enquête de Vivier 1958). 


\section{Un NOUVEL ESSAI DE MORPHOLOGIE EXTERNE COMPARATIVE}

J'ai laissé de côté jusqu'ici la morphologie externe dont il me paraît amplement démontré qu'elle dépend essentiellement des conditions de milieu. Pourtant, les caractères morphologiques sont nécessairement l'expression des possibilités latentes héréditaires de chaque individu. Il était par conséquent indiqué, ayant établi l'existence probable d'au moins six espèces européennes, de vérifier si malgré la difficulté il était possible de distinguer des différences morphologiques ou du moins des tendances particulières à chaque type.

L'ensemble des mensurations que j'ai prises sur des milliers d'individus constitue une énorme documentation dont la publication serait vaine car elle ne mène à rien. Du moins, me semblerait-il futile de baser sur ces documents une pseudosystématique qui aboutirait à une pulvérisation des formes types en sous-espèces et en écotypes, qu'on pourrait multiplier et désigner nommément presque à l'infini.

Dans le tableau 16 je n'ai retenu que quelques mensurations et quelques rapports correspondant à des caractères qui ont été employés pour la systématique du genre. Pour plus de clarté, j’ai arrondi les nombres à l'unité, à 0,5 près ou à 0,1 près, selon les cas. Je rappelle le sens de mes abbréviations:

Long. = longueur du corps, de la pointe du museau à l'extrémité du lobe inférieur de la nageoire caudale.

Branch. = nombre de branchiospines sur le premier arc branchial gauche. Ecailles $=$ nombre d'écailles de la ligne latérale.

écarts = nombre d'écarts interspinaux correspondant à la longueur de la plus grande branchiospine.

$\mathrm{Xp}=$ fonction discriminante, soit $3,5 \mathrm{x}+\mathrm{y}$ (ou $x$ égale le nombre de branchiospines et $y$ le rapport en $\%$ de la plus longue branchiospine à la longueur de la branche inférieure de l'arc branchial).

$3 / 1=$ rapport de la longueur de la tête à celle du corps.

$\mathrm{M} / 3=$ rapport de la hauteur à la longueur de la tête.

$4 / 3=$ rapport du diamètre de l'œil à la longueur de la tête.

$6 / 1=$ rapport de la longueur de la pectorale à celle du corps.

$\mathrm{C} / \mathrm{D}=$ rapport de la hauteur de la nageoire dorsale à sa base.

max./tète = rapport de la longueur de la mâchoire inférieure (soit distance entre la pointe du dentaire et l'angle de la mâchoire inférieure formé par l'angulaire) à la longueur de la tête. 
Tableau 16

Caractères morphologiques

(séries restreintes de 50 individus an maximum)



$1^{\text {re }}$ espèce: $C$. acronius

\begin{tabular}{|c|c|c|c|c|c|c|c|c|c|c|c|c|c|c|}
\hline $\begin{array}{l}(26) \\
(1) \\
47 \\
61 \\
6\end{array}$ & $\begin{array}{l}\text { Storsik } \\
\text { Kilch } \\
\text { Storsik } \\
\text { Kropfer } \\
\text { Storsik }\end{array}$ & $\begin{array}{l}\text { Näckten } \\
\text { Constance } \\
\text { Ismundsjön } \\
\text { Thoune } \\
\text { Storsjön }\end{array}$ & $\begin{array}{c}(36) \\
27 \\
(40) \\
27 \\
44\end{array}$ & $\begin{array}{l}21 \\
21 \\
22 \\
21 \\
22\end{array}$ & $\begin{array}{l}91 \\
82 \\
88 \\
80 \\
89\end{array}$ & $\begin{array}{l}3,1 \\
3,2 \\
3,4 \\
3,9 \\
3,8\end{array}$ & $\begin{array}{r}98 \\
98 \\
101 \\
104\end{array}$ & $\begin{array}{l}24 \\
25 \\
24 \\
29 \\
26\end{array}$ & $\begin{array}{l}18 \\
18,5 \\
18 \\
17,5 \\
18\end{array}$ & $\begin{array}{l}69 \\
73,5 \\
71,5 \\
74,5 \\
71,5\end{array}$ & $\begin{array}{l}19.5 \\
21,5 \\
19,5 \\
23 \\
18,5\end{array}$ & $\begin{array}{l}15 \\
15 \\
16 \\
16,5 \\
16\end{array}$ & $\begin{array}{l}155 \\
171 \\
157 \\
173 \\
153\end{array}$ & $\frac{28}{32}$ \\
\hline & & & 35 & 21,5 & & 3.5 & 101 & 24,5 & 18 & 72 & 20,5 & 15,5 & & 29,5 \\
\hline
\end{tabular}

2e espèce: C. fera

\begin{tabular}{|c|c|c|c|c|c|c|c|c|c|c|c|c|c|c|}
\hline $\begin{array}{l}56 \\
20 p \\
48\end{array}$ & $\begin{array}{l}\text { Maräne } \\
\text { Sandfelchen } \\
\text { Renke }\end{array}$ & $\begin{array}{l}\text { Selentersee } \\
\text { Constance } \\
\text { Riegsee }\end{array}$ & $\begin{array}{l}42 \\
44 \\
31\end{array}$ & $\begin{array}{l}24 \\
25 \\
26\end{array}$ & $\begin{array}{l}90 \\
87 \\
82\end{array}$ & $\begin{array}{l}4,5 \\
4,5 \\
4,9\end{array}$ & $\begin{array}{l}114 \\
114 \\
121\end{array}$ & $\begin{array}{l}28 \\
29 \\
31\end{array}$ & $\begin{array}{l}18 \\
16,5 \\
16,5\end{array}$ & $\begin{array}{l}73 \\
71,5 \\
66,5\end{array}$ & $\begin{array}{l}18,5 \\
18,5 \\
20,5\end{array}$ & $\begin{array}{l}15 \\
14,5 \\
13,5\end{array}$ & $\begin{array}{l}176 \\
168 \\
176\end{array}$ & $\frac{28,5}{27,5}$ \\
\hline & & & 39 & 25 & & 4,6 & 116 & 29 & 17 & 70 & 19 & 14,5 & & 28 \\
\hline
\end{tabular}

\begin{tabular}{|c|c|c|c|c|c|c|c|c|c|c|c|c|c|c|}
\hline $\begin{array}{r}49 \\
15 \\
9 \\
51\end{array}$ & $\begin{array}{l}\text { Palée } \\
\text { Storsik } \\
\text { Schnäpe] } \\
\text { Balchen }\end{array}$ & $\begin{array}{l}\text { Neuchâtel } \\
\text { Landösjön } \\
\text { Kingköbbing } \\
\text { Thoune }\end{array}$ & $\begin{array}{l}41 \\
32 \\
34 \\
33\end{array}$ & $\begin{array}{l}27 \\
29 \\
29 \\
30\end{array}$ & $\begin{array}{l}90 \\
92 \\
82 \\
86\end{array}$ & $\begin{array}{l}5,3 \\
4,9 \\
5,4 \\
5\end{array}$ & $\begin{array}{l}126 \\
130 \\
133 \\
134\end{array}$ & $\begin{array}{l}32 \\
30 \\
31 \\
31\end{array}$ & $\begin{array}{l}16 \\
17,5 \\
17,5 \\
16,5\end{array}$ & $\begin{array}{l}72,5 \\
69 \\
70 \\
72\end{array}$ & $\begin{array}{l}19 \\
20,5 \\
19,5 \\
20,5\end{array}$ & $\begin{array}{l}13,5 \\
15 \\
13,5 \\
14,5\end{array}$ & $\begin{array}{l}150 \\
159 \\
152 \\
160\end{array}$ & $\begin{array}{l}-\overline{29} \\
32 \\
\end{array}$ \\
\hline & & & 35 & 29 & & 5,2 & 131 & 31 & 17 & $\gamma_{1}$ & 20 & 14 & & 30, \\
\hline
\end{tabular}

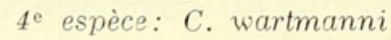

\begin{tabular}{c|l|l|l|l|l|l|l|l|l|l|l|l|l|l|l}
\hline 3 & Powan & Loch Lomond & 28 & 34 & 81 & 6,7 & 151 & 32 & 17 & 69,5 & 23 & 15 & 167 & 31 & 15 \\
50 & Bondelle & Neuchâtel & 32 & 34 & 81 & 7,6 & 156 & 37 & 17 & 69.5 & 22,5 & 14 & 177 & 28,5 \\
$(25)$ & Storsik & Fullsjön & 36 & 36 & 90 & 7,9 & 151 & 35 & 17,5 & 71,5 & 19,5 & 16 & 171 & 26 \\
$6 \mathrm{~B}$ & Skelly & Ullswater & 38 & 37 & 77 & 7,8 & 165 & 34 & 17 & 76,5 & 20 & 15,5 & 175 & 29 \\
36 & Grásik & Revsundsjön & 31 & 37 & 96 & 7,7 & 165 & 36 & 17 & 67 & 21,5 & 14 & 156 & 28 \\
\hline
\end{tabular}

5 espèce: C. macrophthalmus

\begin{tabular}{|c|c|c|c|c|c|c|c|c|c|c|c|c|c|c|}
\hline $\begin{array}{l}(5) \\
22 \\
57 \\
21\end{array}$ & $\begin{array}{l}\text { Pollan } \\
\text { Albock } \\
\text { Renke } \\
\text { Brienzlig }\end{array}$ & $\begin{array}{l}\text { Lough Neagh } \\
\text { Thoune } \\
\text { Riegsee } \\
\text { Brienz }\end{array}$ & $\begin{array}{l}26 \\
38 \\
39 \\
17\end{array}$ & $\begin{array}{l}38 \\
39 \\
42 \\
42\end{array}$ & $\begin{array}{l}80 \\
90 \\
93 \\
81\end{array}$ & $\begin{array}{l}8,8 \\
9,2 \\
9,2 \\
9,8\end{array}$ & $\begin{array}{l}171 \\
176 \\
185 \\
188\end{array}$ & $\begin{array}{l}37 \\
41 \\
38 \\
42\end{array}$ & $\begin{array}{l}18,5 \\
16 \\
19 \\
18\end{array}$ & $\begin{array}{l}66 \\
73,5 \\
69,5 \\
64\end{array}$ & $\begin{array}{l}22 \\
19,5 \\
20,5 \\
24\end{array}$ & $\begin{array}{l}14 \\
14 \\
14,5 \\
15\end{array}$ & $\begin{array}{l}151 \\
155 \\
168 \\
180\end{array}$ & $\begin{array}{l}\frac{33,5}{29} \\
32\end{array}$ \\
\hline & & & 30 & 40 & & 9,3 & 180 & 39,5 & 18 & 68 & 21,5 & 14,5 & & 31,5 \\
\hline \multicolumn{15}{|c|}{$6^{\text {e }}$ espèce: C. albula } \\
\hline \multirow[t]{2}{*}{$\begin{array}{l}(4) \\
58 \\
53\end{array}$} & $\begin{array}{l}\text { Pollan } \\
\text { Kl. Maräne }\end{array}$ & $\begin{array}{l}\text { Lough Erne } \\
\text { Dieksee } \\
\text { Schalsee }\end{array}$ & $\begin{array}{l}26 \\
21 \\
23\end{array}$ & $\begin{array}{l}43 \\
43 \\
44\end{array}$ & $\begin{array}{l}83 \\
80 \\
82\end{array}$ & $\begin{array}{l}12,4 \\
13,4 \\
13,9\end{array}$ & $\begin{array}{l}195 \\
199 \\
202\end{array}$ & $\begin{array}{l}46 \\
48 \\
50\end{array}$ & $\begin{array}{l}18 \\
18 \\
17\end{array}$ & $\begin{array}{l}65,5 \\
64,5 \\
65\end{array}$ & $\begin{array}{l}21 \\
24 \\
22,5\end{array}$ & $\begin{array}{l}15 \\
14 \\
13\end{array}$ & $\begin{array}{l}155 \\
177 \\
169\end{array}$ & $\begin{array}{l}32 \\
34 \\
32\end{array}$ \\
\hline & & & 23 & 43 & & 13,2 & 198 & 48 & 18 & 6.5 & 22,5 & 14 & & 32,5 \\
\hline
\end{tabular}

(Entre parenthèses: mesures sur matériel fixé.) 


\section{Longueur du corps}

Deux espèces seulement paraissent avoir des dimensions relativement constantes: C. wartmanni dont les diverses populations fluctuent grosso modo entre 30 et $40 \mathrm{~cm}$ et $C$. albula dont les capacités de croissance paraissent limitées à des moyennes de 20 à $25 \mathrm{~cm}$.

La première espèce, $C$. acronius, devient presque énorme en Suède, atteignant jusqu'à $44 \mathrm{~cm}$ en moyenne pour la population du Storsjön, tandis que les populations des Alpes semblent végéter et n'atteignent que $27 \mathrm{~cm}$ en moyenne. Les tailles atteintes par les autres espèces sont très variables d'une population à l'autre, celle du lac de Brienz, un macrophthalmus, est comme frappée de nanisme; on peut supposer que sa multiplication est considérable eu égard aux conditions précaires d'existence dans un lac alimenté par l'eau glaciaire, froide et limoneuse de l'Aar. $C$. fera semble être l'espèce dont les potentialités de croissance sont les plus grandes.

\section{Ecailles de la ligne latérale}

On est frappé d'emblée par la différence entre les populations suédoises quelle que soit l'espèce à laquelle elles appartiennent et la plupart de celles des Alpes: toutes les populations du Jämtland accusent une moyenne de 90 ou supérieure à ce nombre, qui n'est atteint dans les Alpes que par quelques contingents. Fonction des conditions écologiques, le nombre des écailles dépend, comme l'a confirmé Svärdson, de la température de l'eau au moment de leur formation chez l'alevin: en eau glacée, les ébauches d'écailles se forment plus nombreuses qu'en eau moins froide. Le nombre d'écailles ne peut done entrer en ligne de compte pour la systématique des Corégones.

Dans un même lac du plateau suisse, comme celui de Neuchâtel, l'espèce qui fraie en profondeur, où la température est sensiblement stable à environ $5^{\circ}$, présente un faible nombre d'écailles (moyenne 81); celle dont les alevins paraissent exposés aux rigueurs des températures hibernales en beine ou au bord du mont, atteint les 90 écailles des populations du nord. Toutes les formes britanniques ont un nombre d'écailles voisin de 80 , conséquence apparente d'un climat océanique plus doux. 
Longueur de la tête relativement à la longueur du corps (3/1)

Il me paraît intéressant que ce rapport, dont les écarts d'une espèce à l'autre sont d'ailleurs faibles, soit équivalent dans $C$. acronius et $C$. albula.

Proportions de la tête (rapport $\mathrm{M} / 3$ )

Une forte valeur de ce rapport signifie une tête relativement haute et courte. C'est donc nettement $C$. acronius qui a la tête la moins allongée relativement à sa hauteur, tandis qu'à l'opposé C. albula présente la tête la plus effilée. Ce rapport peut d'ailleurs exprimer d'une façon générale l'allongement relatif de tout l'individu; il est très faible chez la population naine du lac de Brienz; tandis que l'Albock du lac voisin de Thoune, qui est aussi un macrophthalmus, fournit une des plus fortes valeurs observées.

\section{Grandeur relative de l'œil (rapport 4/3)}

Ce rapport dépend manifestement de la croissance, comme on peut s'en convaincre en considérant les populations de la première espèce: à grande taille correspond un œil relativement petit. C'est probablement pourquoi la population naine du lac de Brienz et la plus petite des petites Marènes, celle du Dieksee, fournissent les indices les plus élevés, voisins de 24. Cependant, il semble que, dans l'ensemble, ce rapport augmente progressivement de la deuxième à la sixième espèce.

Longueur relative de la nageoire pectorale (rapport 6/1)

La nageoire pectorale montre une croissance allomorphique évidente. Elle doit s'accroître progressivement avec l'âge, indépendamment de la rapidité de croissance. Il semble qu'on puisse attribuer à des différences d'âge moyen l'écart notable qui sépare à ce point de vue $C$. acronius de $C$. albula ou même de $C$. lavaretus.

\section{Proportions de la nageoire dorsale (rapport C/D)}

Les fortes valeurs du rapport indiquent des nageoires dorsales relativement hautes et étroites, les faibles valeurs, des nageoires 
courtes et larges. J'ai signalé dans un précédent travail qu'une nageoire allongée et étroite semblait corrélative de la vie en profondeur. Pour autant que je sois bien renseigné, ce tableau confirme le fait: ont en commun un rapport de 170 ou plus, le Kilch et le Kropfer (C. acronius), la grande Marène du Selentersee et le Renke du Chiemsee ( $C$. fera), la Bondelle ( $C$. wartmanni) et le Brienzlig (C. macrophthalmus), populations appartenant à des espèces différentes mais qui ont en commun la particularité de vivre de préférence en profondeur. Peut-être en est-il de même de la petite Marène du Dieksee et du Skelly, par exemple ? Si cette corrélation, allongement de la nageoire dorsale et vie en profondeur est bien authentique, on peut se demander quelle est la cause et quel est l'effet. J'aurai garde de me prononcer, mais je suppose qu'il s'agit d'un caractère adaptatif non héréditaire.

\section{Longueur relative du maxillaire inférieur (rapport max./tête)}

Je n'ai malheureusement pas mesuré au début de mes recherches la longueur du maxillaire parce que je m'en tenais aux mensurations de Steinmann. Cette mesure m'est apparue utile au cours des travaux; elle s'est en effet révélée une des plus intéressantes, en particulier comme caractère d'appoint pour distinguer $C$. wartmanni de $C$. macrophthalmus, ce dernier ayant le museau nettement plus allongé. $C$. albula, grâce à son menton saillant, fournit la valeur la plus forte de ce rapport, qui devrait être repris de façon systématique pour être jugé à sa juste valeur.



On peut conclure de cette rapide analyse de quelques caractères de morphologie externe que les proportions du corps telles qu'elles sont exprimées au tableau 16 ou tout autres valeurs comme celles que nous avons discutées précédemment (Dottrens et Quartier 1949) sont inutilisables pour un classement systématique des populations.

Cette conclusion n'est évidemment pas neuve, mais elle ne paraît pas avoir été admise par les auteurs qui prennent ces mesures en considération pour aboutir à des subdivisions qui sont sans intérêt pratique ni valeur systématique. En revanche, il serait 
intéressant, en se basant sur les données de la biométrie, d'examiner point par point quelles sont les particularités biologiques du milieu qui modifient dans un sens ou dans l'autre les proportions moyennes observées dans une espèce donnée. Les commentaires précédents peuvent ètre considérés comme des hypothèses de travail pour des études et observations écologiques approfondies.

\section{Correspondance AVEc Le Système de Järvi (1928)}

La correspondance de cette classification avec celle de Järvi (1928) est remarquable, si on considère que celle-ci est en partie fondée sur des populations de Laponie et celle-là, principalement sur les Corégones alpins. Voici cette correspondance:

10 . acronius Smitt éc. 3,5

$2^{\circ}$ C. fera Jurine

Br. 24 à 25

éc. 4,5

30 C. lavaretus (L.)

Br. 28 à 29

éc. 5 à 5,5

$4^{\circ}$ C. wartmanni Bloch.

Br. 35 à 36

éc. 7,5

$5^{\circ}$ C. macrophthalmus Nüsslin Br. 40

éc. 9 à 9,5

$6^{\circ}$ C. albula (L.)

Br. 43

éc. 13 à 13,5
JäRVI (1928)

C. fera Jurine

$=$ Inari Flussmaräne ( $f$. inarensis)

Br. 21,6 long. relat. branch.: 7,8

C. holsatus Thien.

$=$ Lehtisiika Maräne ( $f$. anarensis) Br. 24,1 long. relat. branch.: 6,8

C. lavaretus L.

Lappländische Maräne (f. lapponica) Inari

Br. 28,7

Wandermaräne (f. typica Thien.)

Br. 29,8

long. relat. branch.: 10,6

C. wartmanni Bl.

Riika Maräne (f. borealis)

Br. 33 à 34

long. relat. branch.: 8,2 à 8,9

Murokas Maräne

Br. 35 à 38,7

long. relat. branch.: 8,8 à 9,9

C. macrophthalmus Nüssl.

Kleine Binnensee Maräne

Br. 42,5

long. relat. branch.: 8,1

C. generosus Peters

Edelmaräne $=$ Grosse Binnensee

Maräne (f. aspia Smitt)

Br. 45,8 à 49,5

long. relat. branch.: 6,9 à 8,9 
On n'observe qu'une seule divergence importante. Järvi donne le nom de $C$. fera Jurine à la première espèce alors qu'il est évident que la Féra de Jurine, comme la grande Marène du Selentersee (C. holsatus) appartiennent au type Sandfelchen, donc à la deuxième espèce.

$C$. holsatus tombe de ce fait en synonymie avec $C$. fera Jurine et la première doit s'appeler $C$. acronius Smitt.

JäRvi exprime la longueur relative des branchiospines en divisant la longueur totale de l'arc par la longueur de la plus longue branchiospine. La valeur indiquée est done d'autant plus faible que les branchiospines sont relativement plus longues. Je ne m'explique pas comment il se fait que les différences entre espèces ne soient pas plus évidentes, et encore moins que cette valeur atteigne son maximum chez $C$. lavaretus.

Je n'ai pas rencontré, au cours de mes recherches, une seule population appartenant à l'espèce $C$. generosus Peters.

\section{Comparaison avec le système de Berg}

Je me base pour cette comparaison sur le système de Berg tel qu'il figure page 378 dans le travail de WAgLER (1941).

J'ai déjà dit pourquoi l'opposition $C$. albula- $C$. lavaretus me paraît erronée. Si on accorde le rang spécifique aux sous-espèces de BERG on peut établir une certaine correspondance moyennant diverses retouches:

C. l. wartmanni est divisé, pour l'Europe moyenne, 25 nationes qui sont purement théoriques. Une bonne partie d'entre elles sont artificielles, l'auteur ayant repris les subdivisions de FAtio en y ajoutant encore, et en fondant schématiquement une natio pour chaque lac sans se préoccuper de la nature véritable des populations. C'est ainsi qu'il admet dans le lac de Constance à la fois $C$. $l$. wartmanni et $C$. l. wartmanni natio macrophthalmus, deux nationes différentes dans le même lac pour la même sous-espèce !

Il est évident que wartmanni doit être scindé en deux sousespèces (en deux espèces dans le système adopté dans le présent travail) :

$$
\text { C. l. wartmanni }\left\{\begin{array}{l}
\text { C.l. wartmanni } \\
\text { C.l. macrophthalmus }
\end{array}\right.
$$


Les 25 nationes se répartissent entre les deux groupes ou ne sont que des mélanges ou des populations plus ou moins altérées.

Sous C.l. fera Berg nomme 12 nationes qui sont en réalité des populations de pureté variable et qui se subdivisent aussi en deux groupes:

$$
\text { C. l. fera }\left\{\begin{array}{l}
\text { C. l. fera } \\
\text { C.l. lavaretus }
\end{array}\right.
$$

Mis à part C.l. acronius qui subsiste, il reste encore dans le système de BERG quelques sous-espèces qui tombent en synonymie. Ce sont:

C. l. holsatus tombe en synonymie avec C.l. fera.

C. l. maraena qui n'est pas une forme alpine $=C$. l. fera.

C.l. sulzeri, créations de Fatio pour des populations actuellement disparues et qui étaient probablement des C. l. lavaretus.

C. l. hoferi Berg, du Chiemsee, que j'ai rapporté à Coregonus fera.

C. l. hiemalis Jurine, du Léman, forme disparue qui était probablement un produit artificiel ou hybride.

C. l. bezola Fatio, du lac du Bourget, absolument comparable à la précédente.

\section{Résumé et Conclusions}

L'étude de quelque 60 populations de Corégones provenant de divers lacs de la région alpine, du nord de l'Allemagne, du Jämtland en Suède, du Danemark, de la Grande-Bretagne et de l'Irlande du Nord, et celle d'une population importée en Auvergne, aboutit à distinguer six espèces caractérisées surtout par le nombre et la longueur relative des branchiospines du premier arc branchial. Dans la mesure du possible, ces populations ont été examinées et mesurées sur place.

Ces six espèces sont:

C. acronius Rapp: 20 à $22 \mathrm{Br}$. et 3,5 écarts interspinaux en moyenne.

C. fera Jurine: 24 à $25 \mathrm{Br}$. et 4,5 écarts interspinaux en moyenne.

C. lavaretus (L.): 29 à $30 \mathrm{Br}$. et 5,5 écarts interspinaux en moyenne. 
C. wartmanni Bloch: 35 à $36 \mathrm{Br}$. et 7,5 écarts interspinaux en moyenne.

C. macrophthalmus Nüsslin: $40 \mathrm{Br}$. et 9 à 10 écarts interspinaux en moyenne.

C. albula (L.): $43 \mathrm{Br}$. et 13 écarts interspinaux en moyenne.

Une fonction discriminante, $\mathrm{X} p=3,5 x+y$, a été calculée qui prend en considération le nombre de branchiospines du premier arc $(x)$ et la longueur relative des branchiospines exprimée par le rapport en $\%$ de la plus grande d'entre elles à la longueur de la branche inférieure de ce premier arc (ce rapport $=y$ ).

La plupart des populations examinées ont montré des degrés divers de mélange, brassages, introgressions et hybridations qui semblent pour la plupart consécutifs aux interventions humaines. L'examen des principales mensurations sur les populations les plus pures des diverses espèces confirme la difficulté insurmontable de distinguer des caractères morphologiques valables pour la systématique. Ce qui revient à dire que les subdivisions en sous-espèces ou en types écologiques, dans l'état actuel de nos connaissances, ne sauraient être que subjectives et arbitraires.

\section{RÉFÉRENCES}

Blis, C. I. 1935. The calculation of the dosage-mortality curve. Ann. applied Biol. 22: 134-167.

Dottrens, E. 1950. Le Corégone actuel du Léman. Rev. suisse Zool. 57 : 689-813.

- 1953. Contribution à la connaissance du Weissfelchen de l'Untersee (genre Coregonus Salmonid.). Rev. suisse Zool. 60: $452-461$.

- 1955. Acclimatation et hybridation de Corégones. Rev. suisse Zool. 62, fasc. suppl.: 101-118.

- 1958. Sur les Corégones de Grande-Bretagne et d'Irlande. XVe Congrès de Zool., sect. V, 3 p.

- et Quartier, A. 1949. Les Corégones du lac de Neuchâtel. Etude biométrique. Rev. suisse Zool. 56:689-730.

Einsele, W. 1943. Uber das Wachstum der Coregonen im Voralpengebiet insbesondere über das Verhältnis von Schuppenlängenwachstum. Z. Fisch. u. d. Hilfswiss. 41: 23-45.

Fatio, V. 1890. Faune des Vertébrés de la Suisse. Vol. 5. Histoire naturelle des poissons. II e partie. Georg, Genève et Bâle. 
Fisher, R. A. 1946. Statistical Methods for Research Workers. Parag. 49.2, 10th edition. Oliver and Boyd, Edinburgh.

Forel, F. A. 1904. Le Léman, monographie limnologique. Rouga, Lausanne, 3 vol.

Gasowska, M. 1956. Research on an reciprocal cross-breeding of Coregonus lavaretus maraenoides Poliakow and Coregonus albula (L.) (en polonais, résumé anglais). Polskii Archiv. Hydrobiol.. III (XVI): 253-268.

Järvi, T. H. 1928. Uber die Arten und Formen der Coregonen s.str. in Finnland. Acta zool. fenn. 5, 259 p.

Kennedy, W. A. 1943. The Whitefish. Coregonus clupeaformes (Mitchill) of the Lake Opeongo, Algonquin Park, Ontario. Publ. Ontario Fish. Res. Labor. 51: 23-66.

Lamoтte, M. 1948. Introduction à la Biologie quantitative. Masson, Paris, 369 p.

Monti, R. 1929. Biologia dei Coregoni nei laghi italiani. Riv. Biol. Milano II : 317-335, 709-729, 733-764.

Nümann, W. 1938. Kritische Bemerkungen zu Waglers Aufsatzserie über die Bewirtschaftung der Renkenseen des Vorderalpengebiets. Allg. Fischrei-Z. 63: 100-104.

Sonneborn, T. M. 1957. Breeding systems, reproductive methods, and species problem in Protozoa in The Species Problem. A. A. Adv. Sc., Wash. D. C. Publ. No. 50.

Spillmann, J. 1958. Sur deux poissons des collections du Muséum, témoins de l'existence au XIX $X^{\mathrm{e}}$ siècle, dans le lac Léman, de Corégones du type "dispersus». Bull. Muséum, Paris, 2 e sér. 30:144-145.

Steinmann, P. 1948. Der Weissfelchen des Bodensees und die Frage der Artbildung im Felchengeschlecht. Z. Hydrol., Aarau 10/4: 3-12.

- 1950. Ein neues System der mitteleuropäischen Coregonen. Rev. suisse Zool. 57: 517-525.

- 1950. (II.) Monographie der schweizerischen Coregonen. Birkhäuser, Bâle, $490+$ pp 54 à 191. Sonderabdr. Schw. Z. Hydrol. 12, fasc. 1 et 2 et 13, fasc. 1.

Svärdson, G. The Coregonid Problem. Inst. Fresh-Water Res. Drottningholm. 1949, rep. 29: 89-101; 1950, rep. 31: 151-162; 1951, rep. 32: 79-166; 1952, rep. 33: 204-232; 1953, rep. 34: 142-166; 1957, rep. 38: 267-356.

- 1958. Systematics of To-Day in Proceedings of a symposium held at the Univ. of Uppsala. Uppsala Univ. Arsskrift 6: 231-239.

Vivier, P. 1958. Un exemple d'acclimatation ancienne: les Corégones des lacs du Massif central. Ann. Stat. centr. Hydrol. appl. 7: 241-250. 
Wagler, E. 1937. Die Systematic der Voralpencoregonen. Int. Rev. ges. Hydrob. Hydrol. 35: 345-446.

- 1941. Die Lachsartigen: Coregonen in Handbuch der Binnenfischerei. 3: 371-501.

- 1950. Die Coregonen in den Seen des Voralpengebietes XI Herkunftund Einwanderung der Voralpencoregonen. Veröff. zool. Staatsvers. München 1:3-62.

Wunder, W. 1939. Die "Hungerform" und die "Mastform" des Karpfens (Cyprinus carpio L.). Z. Morph. Ökol. Tiere 35: 594-614.

Zandt, F. 1937. Zur Biologie des Blaufelchens (Coregonus xartmanni Bl.) des Bodensees. Inter. Rev. Hydrob. 36/1-2: 138-169.

- 1950. Idem, 2 Beitrag: Verteilung der Altersklassen im Bodensee. Arch. Hydrol. Stuttgart 43: 459-496. 


\section{$2 \mathrm{BHL}$ Biodiversity Heritage Library}

Dottrens, E. 1959. "Systématique des Corégones de I'Europe occidentale, basée sur une étude biométrique." Revue suisse de zoologie 66, 1-66. https://doi.org/10.5962/bhl.part.75206.

View This Item Online: https://www.biodiversitylibrary.org/item/126484

DOI: https://doi.org/10.5962/bhl.part.75206

Permalink: https://www.biodiversitylibrary.org/partpdf/75206

\section{Holding Institution}

Smithsonian Libraries

\section{Sponsored by}

Biodiversity Heritage Library

\section{Copyright \& Reuse}

Copyright Status: In Copyright. Digitized with the permission of the rights holder

Rights Holder: Muséum d'histoire naturelle - Ville de Genève License: http://creativecommons.org/licenses/by-nc-sa/3.0/

Rights: https://www.biodiversitylibrary.org/permissions/

This document was created from content at the Biodiversity Heritage Library, the world's largest open access digital library for biodiversity literature and archives. Visit BHL at https://www.biodiversitylibrary.org. 